FEEDBACK CONTROL OF A HIGH LEVEL UPPER EXTREMITY NEUROPROSTHESIS

\author{
by \\ DIMITRA BLANA \\ Submitted in partial fulfillment of the requirements \\ for the degree of Doctor of Philosophy \\ Dissertation Adviser: Dr. Robert F. Kirsch \\ Department of Biomedical Engineering \\ CASE WESTERN RESERVE UNIVERSITY
}

May, 2008 


\section{CASE WESTERN RESERVE UNIVERSITY SCHOOL OF GRADUATE STUDIES}

We hereby approve the thesis/dissertation of

Dimitra Blana

\begin{tabular}{|c|c|}
\hline & Dimitra Blana \\
\hline & candidate for the $\mathrm{PhD}$ degree *. \\
\hline (signed)_ & $\begin{array}{c}\text { Robert F. Kirsch } \\
\text { (chair of the committee) }\end{array}$ \\
\hline & Patrick E. Crago \\
\hline & Musa L. Audu \\
\hline & Antonie van den Bogert \\
\hline & Wyatt S. Newman \\
\hline (date) & March $17^{\text {th }} 2008$ \\
\hline
\end{tabular}

*We also certify that written approval has been obtained for any proprietary material contained therein. 


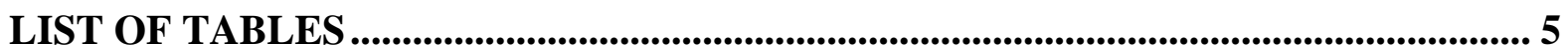

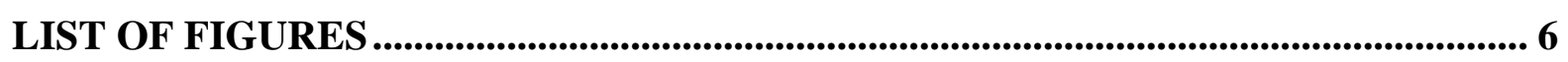

ACKNOWLEDGEMENTS ................................................................................... 9

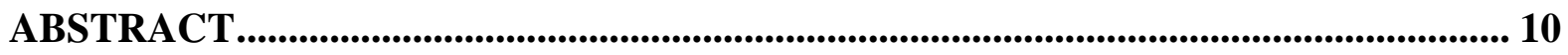

CHAPTER 1: INTRODUCTION............................................................................. 12

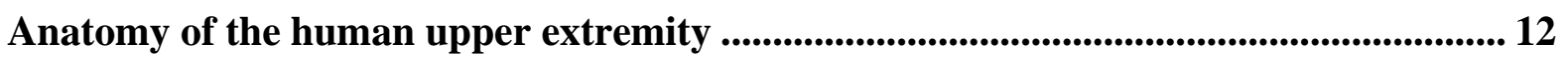

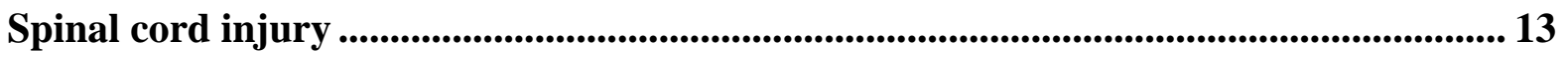

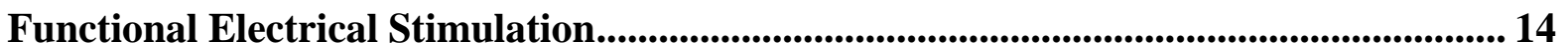

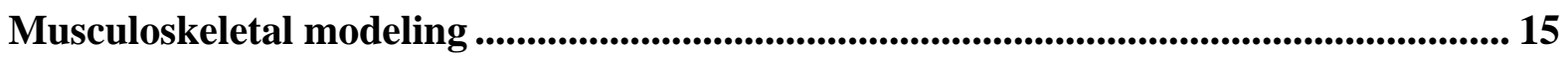

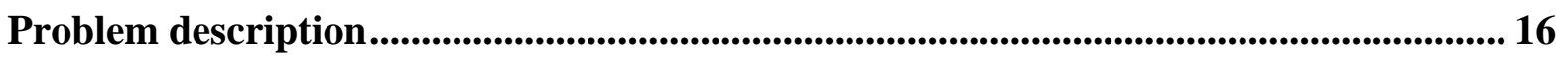

Summary of research............................................................................................................................... 17

CHAPTER 2: A MUSCULOSKELETAL MODEL OF THE UPPER EXTREMITY FOR USE IN THE DEVELOPMENT OF NEUROPROSTHETIC SYSTEMS ............ 19

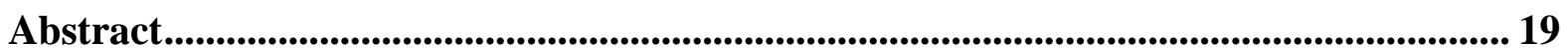

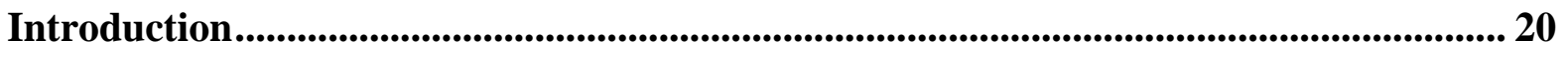

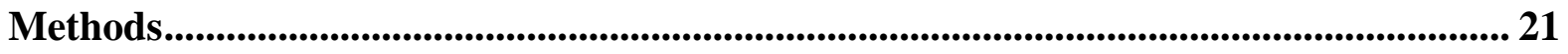

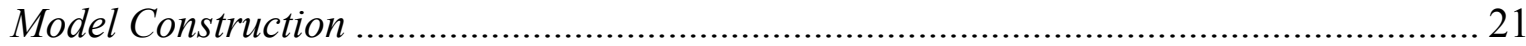

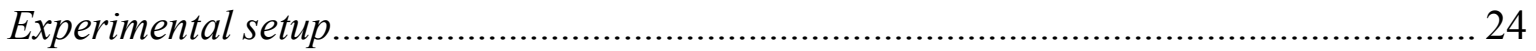

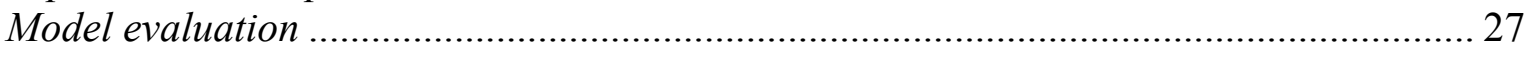

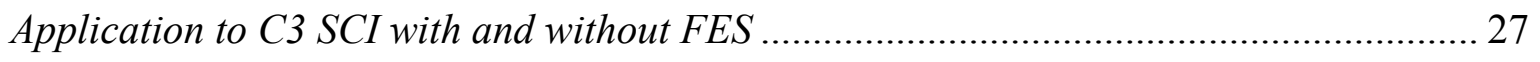

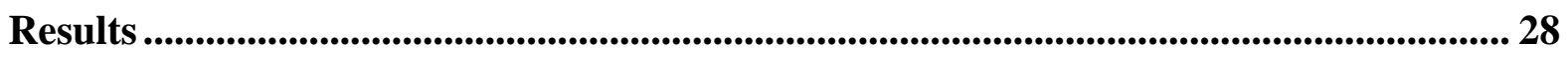

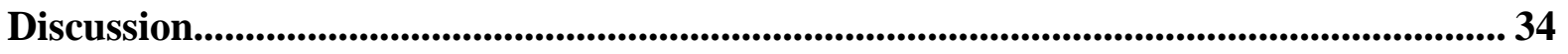

CHAPTER 3: MODEL-BASED SELECTION OF MUSCLE AND NERVE CUFF ELECTRODES FOR A HIGH TETRAPLEGIA NEUROPROSTHESIS ................... 38

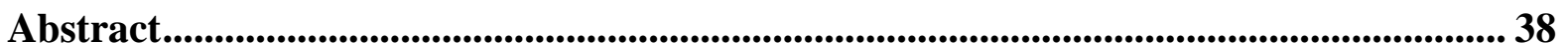


Introduction.................................................................................................................................. 39

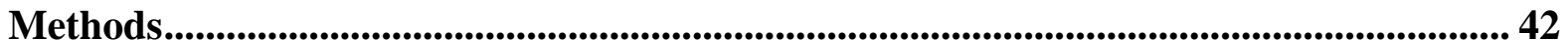

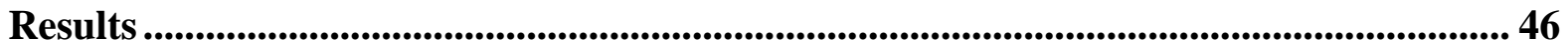

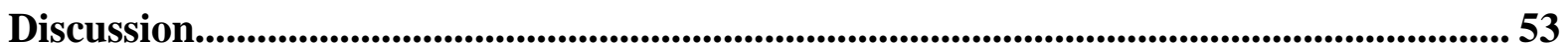

CHAPTER 4: COMBINED FEEDFORWARD AND FEEDBACK CONTROL OF A REDUNDANT, NON-LINEAR, DYNAMIC MUSCULO-SKELETAL SYSTEM........ 57

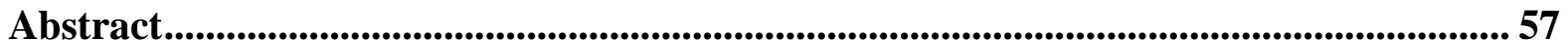

Introduction.......................................................................................................................................... 57

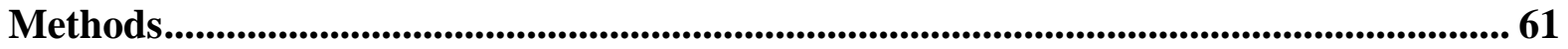

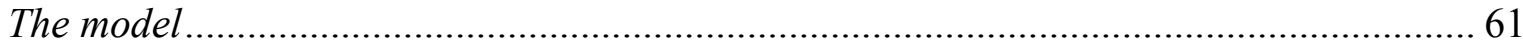

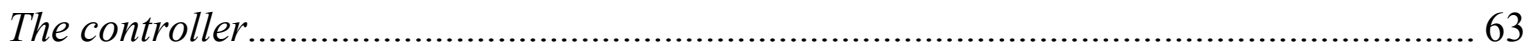

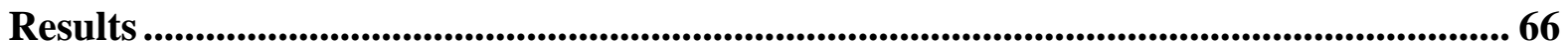

Discussion.......................................................................................................................................... 73

CHAPTER 5: A SHOULDER AND ARM CONTROLLER FOR TRAJECTORY TRACKING AND STABILITY .................................................................................... 76

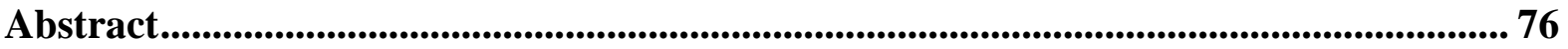

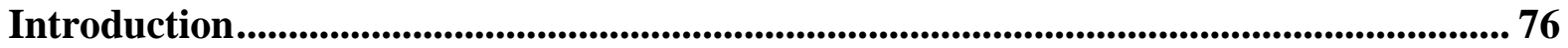

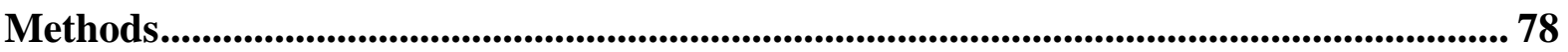

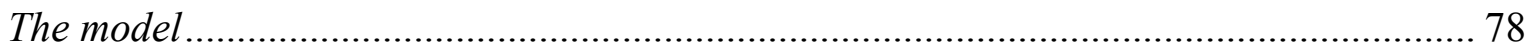

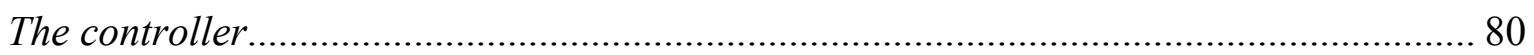

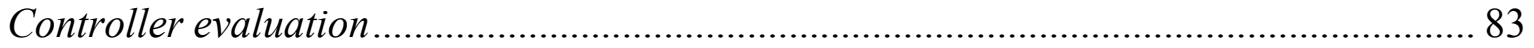

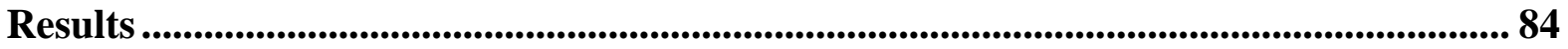

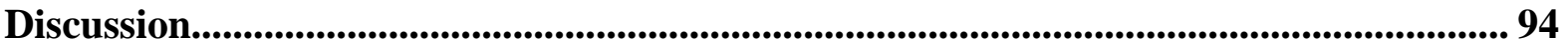

CHAPTER 6: ADAPTIVE CONTROL USING CO-CONTRACTION OF ANTAGONISTIC MUSCLES................................................................................................. 97

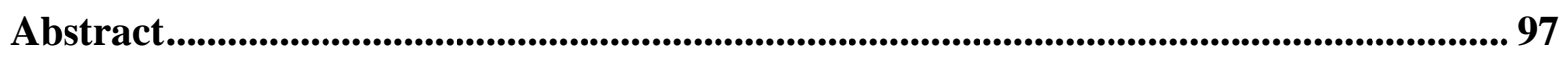

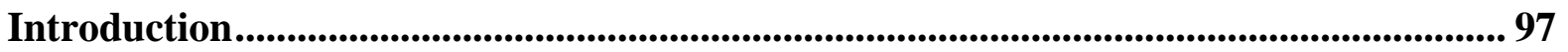

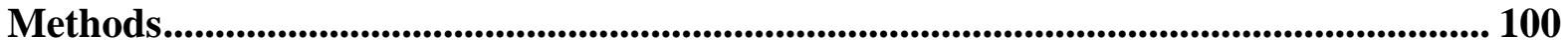

The model ............................................................................................................ 100 
Controller.

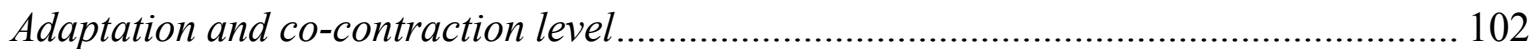

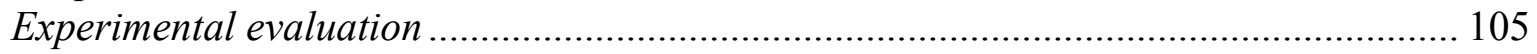

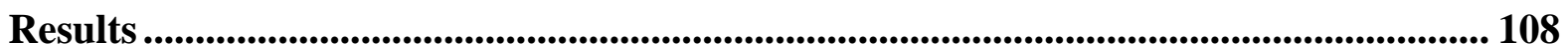

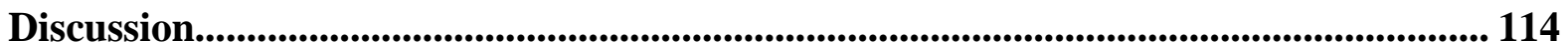

CHAPTER 7: PERFORMANCE OF A FEEDFORWARD-FEEDBACK FES CONTROLLER IN AN INDIVIDUAL WITH HIGH TETRAPLEGIA....................... 117

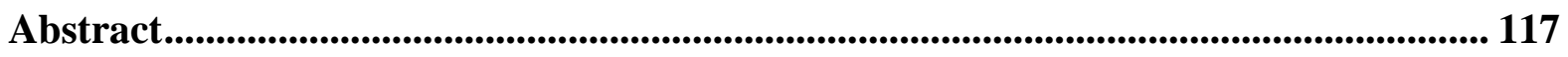

Introduction...................................................................................................................................... 117

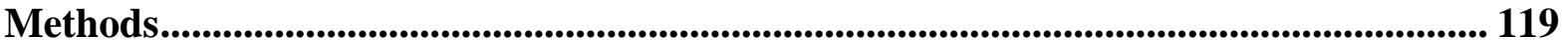

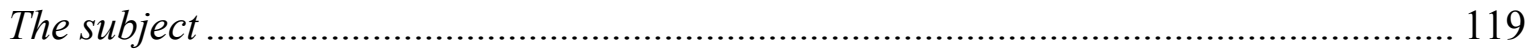

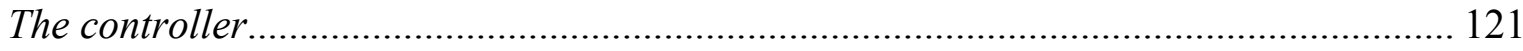

Controller evaluation …………………………………….................................... 125

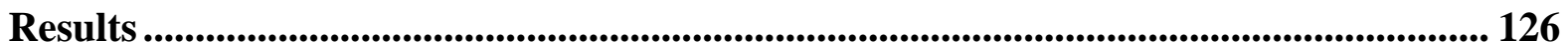

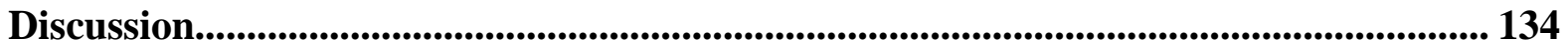

CHAPTER 8: CONCLUSIONS ......................................................................................... 138

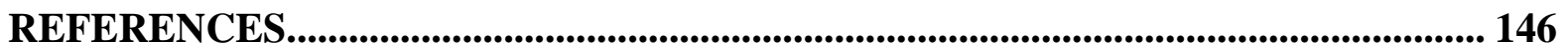




\section{List of Tables}

Table 2-1: The muscles included in the model....................................22

Table 2-2: Electrodes used to measure EMG signals..............................26

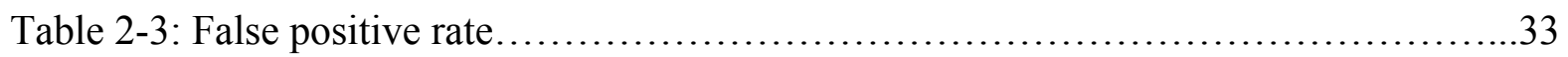

Table 3-1: Nerves and muscles candidate for stimulation.............................45

Table 3-2: Success rate of different electrode sets for every simulated movement..........48

Table 3-3: The optimal electrode set...........................................50

Table 3-4: The success rate of the optimal electrode set for every movement..............50

Table 4-1: The PID gains used in the feedback part of the controller....................65

Table 5-1: The PD coefficients for the feedback controller...........................82

Table 6-1: Parameters of the original model modified to simulate a different individual....100

Table 7-1: The stimulation channels............................................ 120 


\section{List of Figures}

Figure 2-1: Experimental setup............................................... 24

Figure 2-2: EMG recording, processing, and comparison to model-predicted activations....29

Figure 2-3: Serratus anterior, supraspinatus, and infraspinatus signals..................... 30

Figure 2-4: Biceps and triceps signals............................................

Figure 2-5: Average cross-correlation between EMG and model-predicted activations......32

Figure 2-6: Maximum humeral elevation achieved in four cases........................34

Figure 3-1: Inputs and outputs of the model, and calculation of success rate..............47

Figure 3-2: The effect of different selective and non-selective nerve cuff electrodes.........52

Figure 4-1: The two-degree of freedom, six-muscle model..........................61

Figure 4-2: The combined feedforward and feedback controller scheme.................63

Figure 4-3: An example of the performance of the FF, FB and FF+FB controllers.........67

Figure 4-4: The muscle activations calculated for the same movement...................68

Figure 4-5: The mean RMS error using the three controllers..........................69

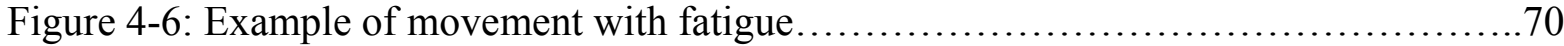

Figure 4-7: The mean RMS error across all trials with fatigue......................... 71

Figure 4-8: Example of the controller performance in the presence of external forces.......72

Figure 4-9: The mean RMS error for all trials with external forces......................72

Figure 5-1: The controller design $\ldots \ldots \ldots \ldots \ldots \ldots \ldots \ldots \ldots \ldots \ldots \ldots \ldots \ldots \ldots \ldots \ldots \ldots \ldots \ldots 1$

Figure 5-2: Example of goal-oriented movement using the static feedforward controller....85

Figure 5-3: The same movement using the dynamic feedforward controller...............86

Figure 5-4: The same movement using the feedback controller......................87 
Figure 5-5: Same example with the combined dynamic FF and FB controller..............88

Figure 5-6: Comparison of the performance of the static and dynamic FF controllers........89

Figure 5-7: Summary of errors from trials with the combined FF+FB controller............90

Figure 5-8: The GH stability index for all trials with the combined FF+FB controller.......91

Figure 5-9: Example of external force without GH stability correction....................92

Figure 5-10: The same example with GH stability correction........................93

Figure 5-11: The GH stability index for all trials with and without the stability correction...94

Figure 6-1: The controller design, including the adaptation scheme.................... 101

Figure 6-2: Geometrical representation of the co-contraction level.....................104

Figure 6-3: The original controller applied to the original and modified model............108

Figure 6-4: Feedforward control with or without the co-contraction level as an input......109

Figure 6-5: Comparison of the performance of different feedforward controllers..........110

Figure 6-6: Effect of different co-contraction levels used by the FF-FB controller..........111

Figure 6-7: Example of data collection from the subject for training of the FF controller...112

Figure 6-8: Example of an elbow movement performed by the subject..................113

Figure 6-9: Mean error of the FF controllers with and without the co-contraction input.....114

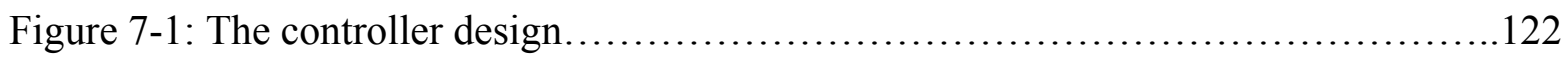

Figure 7-2: Elbow sinusoidal movement with the FF, FB and FF-FB controllers..........127

Figure 7-3: Error summary for the elbow FF, FB and FF-FB controllers.................128

Figure 7-4: Sinusoidal inputs of different frequencies.............................. 129

Figure 7-5: The error for movements of four different frequencies.................... 130

Figure 7-6: Example of elbow movements with step inputs........................ 130

Figure 7-7: The error at the goal of the movement............................... 
Figure 7-8: Example movement for the shoulder and elbow angles....................132

Figure 7-9: Summary of the shoulder-elbow controller performance.....................133

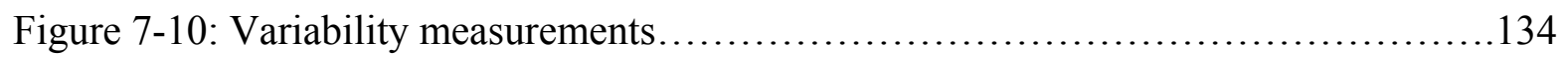




\section{Acknowledgements}

I would like to thank Dr. Robert Kirsch for his enthusiasm and inspiration, and for giving me the opportunity to work on such an exciting project. I am grateful to the rest of my PhD committee members: Drs. Audu, van den Bogert, Crago and Newman for their guidance and invaluable feedback. I would also like to thank my research subjects, whose participation made this study possible.

I would like to acknowledge Andy Cornwell, Juan Gabriel Hincapié, Susy Parra, Katie Polasek and Joris Lambrecht for their help and friendship, and Ed Chadwick for absolutely everything.

Finally, I would like to thank my mum, dad, Irini and Angeliki for the continuous

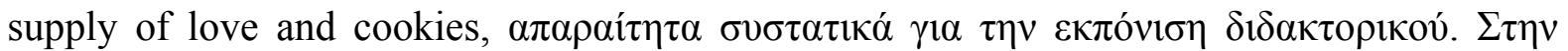

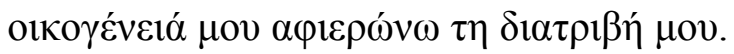




\title{
Feedback Control of a High Level \\ Upper Extremity Neuroprosthesis
}

\author{
Abstract \\ by \\ DIMITRA BLANA
}

The main focus of this study is controller design using a model-based approach, and implementation in a high level upper extremity neuroprosthesis. This is a system that aims to restore upper limb motor function to individuals who have suffered a spinal cord injury at the highest cervical level (C1-C4), and thus have lost voluntary control of most of the shoulder and arm muscles. To determine the optimal electrode set for this system, given the limited number of available stimulating channels, a musculoskeletal model of the upper extremity was used to run a large number of simulations and evaluate all the candidate electrode sets. The model was customized to represent a tetraplegic subject with an FES system, and the optimal electrode set was the one that allowed the model to successfully perform the largest number of movements. Subsequently, a combined feedforward-feedback controller approach was developed and tested first in a simplified arm model with six muscles and two degrees of freedom. In spite of its small scale, the model included an accurate muscle model, redundancy in the number of muscles, and kinematic coupling. It demonstrated the excellent trajectory tracking performance of the controller, even in the presence of considerable muscle fatigue and external forces. Next, the same design, augmented by a controller for shoulder 
stability was tested in a large-scale arm model with 22 muscles and five degrees of freedom, including a complete glenohumeral joint. In addition to its tracking ability, this controller was able to ensure shoulder stability in all cases of external disturbances. Finally, the controller was implemented in one subject with high level tetraplegia, and an adaptation method was developed to adjust the controller parameters according to the characteristics of the specific individual. In order to handle the redundancy of the upper limb, adaptation was performed using co-contraction of antagonistic muscles, and activation patterns among shoulder stabilizing and moving muscles. When tested in the shoulder and elbow of the subject, the controller achieved adaptation and allowed the execution of the desired movements. 


\section{Chapter 1: Introduction}

\section{Anatomy of the human upper extremity}

The human upper extremity, excluding the hand, consists of five bones: clavicle, scapula, humerus, ulna and radius. The thorax, clavicle and scapula create a closed chain, through the sternoclavicular joint, between the thorax and clavicle, acromioclavicular joint, between the clavicle and scapula, and the gliding of the medial border of the scapula on the thorax, in the so-called scapulothoracic gliding plane (van der Helm 1994). The glenohumeral joint is a ball-and-socket joint between the head of the humerus and the shallow glenoid cavity of the scapula. The elbow joint is a hinge between the humerus and ulna, and allows the movement of flexion-extension. Finally, the radio-ulnar joint is actually two separate pivot joints, the proximal and distal, which allow the ulna and radius to rotate about each other.

There are a large number of muscles in the upper extremity, most of them crossing multiple joints. Muscles such as the levator scapulae, which originates on the thorax and attaches on the scapula, cross two joints. Others, running from the thorax to the humerus, such as the latissimus dorsi, cross three joints. Many muscles have large attachment sites: the serratus anterior originates on the surface of the eight or nine upper ribs. Some muscles, such as the rhomboid, have almost straight lines of action; others wrap around the surface of bones, such as the deltoid around the humeral head. This variety in shapes and sizes makes it difficult to determine each muscle's direction of pull and thus its function in any given arm position. 
A unique characteristic of the shoulder is its great flexibility, which comes at the cost of reduced stability. As already mentioned, the glenohumeral joint is a shallow joint, which permits a wide range of motion, but at the same time, is prone to dislocation, if the muscles that surround it do not hold it in place. Displacement is avoided by compression of the humeral head against the glenoid, mainly achieved through the action of the rotator cuff muscles: supraspinatus, infraspinatus, subscapularis and teres minor. Thus, proper activation of these muscles is critical for the function of the upper limb.

\section{Spinal cord injury}

Approximately 255,000 people in the United States have suffered a spinal cord injury, and around 12,000 people sustain new injuries every year. The average age at injury is 39.5 years, and the life expectancy continues to increase: a twenty year old person can expect to survive an additional 23.8 to 53.0 years, depending on the level of injury (Model Spinal Cord Injury System, January 2008).

A spinal cord injury is classified as tetraplegia if the injury occurred in one of the eight cervical segments of the spinal cord, or paraplegia if it occurred in the thoracic, lumbar, or sacral regions. The spinal cord consists of tracts that transmit sensory information from the body to the brain and motor information from the brain to the body. Injury to the spinal cord results in interruption in the transmission of this information. The higher the level of injury,

the more extended the paralysis and sensory loss. At very low lumbar or sacral levels, only bladder and bowel function might be affected, but at the opposite end (i.e. high cervical level) permanent assisted ventilation may be required (Donovan in Stolov and Clowers, 1981). With respect to motor function, a spinal cord injury at the highest cervical levels (C1- 
C4) results in loss of all voluntary control below the neck, with the possible exception of shoulder shrug, produced by the upper trapezius muscle (Kirsch et al. 2001).

\section{Functional Electrical Stimulation}

Neuroprosthetic systems restore function after spinal cord injury using a combination of functional electrical stimulation (FES) and reconstructive surgeries, such as tendon transfers (Keith and Lacey 1991). Currently, neuroprostheses are used to restore bladder and bowel function (Creasy et al. 2001), assist in respiration (Glenn and Phelps 1985), and standing in paraplegia (Davis et al. 2001). In mid-cervical level injuries (C5-C6), they are used to restore hand grasp (Handa and Hoshimiya 1987; Peckham et al. 1988; Popovic et al. 1999; Keith et al. 1988), and improve elbow flexion-extension (Crago et al. 1998) and forearm pronation-supination (Lemay et al. 1996).

FES systems consist of a controller that calculates the muscle excitations needed for a particular task, a stimulator, and electrodes that deliver the stimulation to the appropriate paralyzed muscles. The FES controller has a difficult task due to the large number, redundancy and highly non-linear nature of the muscles, and the complicated interaction between muscles and joints typically seen in biomechanical systems.

Different control strategies are used in neuroprosthetic systems. Feedforward control is commonly used in clinical practice (Jonic and Popovic 1997; Abbas and Triolo 1997). The output of this type of controller depends only on the user command, and not on the system performance. The advantage of this design is that it does not need sensors to measure the system output, but the disadvantage is that it is unable to make corrections if the actual movement deviates from the desired movement. Feedback control uses sensors to monitor 
the system output, so it can correct for errors caused by external disturbances and muscle fatigue (Crago et al. 1996). The use of feedback control is limited in clinical practice due to the need for body-mounted sensors (Chizeck et al. 1988). For the FES systems developed to date, which target low level tetraplegia or paraplegia, feedback is not necessary because the user typically has enough remaining voluntary function to overcome system errors.

\section{Musculoskeletal modeling}

A musculoskeletal model is a mathematical description of a musculoskeletal system. A forward-dynamic model uses muscle activations and external loads as inputs, and calculates the resulting joint angles. An inverse-dynamic model uses joint angles and external loads as inputs, and outputs the required muscle activations. Musculoskeletal models are used to investigate motions, and the muscle forces involved in them (Veeger et al. 1997), or analyze and aid in the treatment of movement abnormalities (Delp 1995). They can also evaluate and suggest ways to enhance performance in sports (Allinger and van den Bogert 1997).

In the area of FES, modeling has been mostly used in the lower extremity, to investigate the conditions for stable sitting (Wilkenfeld et al. 2006), and standing (Kim et al. 2006). Kirsch et al. used a musculoskeletal model of the upper extremity to examine the effect of different muscles in arm motion and stability, and determine the muscles that would be the most effective targets for FES (Kirsch et al. 2001). Modeling is not used more widely

for the development of upper extremity FES controllers because most models of the upper extremity are inverse (Karlsson and Peterson 1992; Hogfors et al. 1987; van der Helm 1994; Charlton and Johnson 2000). Even though inverse modeling is a useful tool in the evaluation 
of different interventions (Kirsch et al. 2001) and controller design (Norgaard et al. 2000), a forward model is necessary as a substitute for the human arm in the testing phase of the neuroprosthesis development.

\section{Problem description}

An implanted neuroprosthesis for restoration of motor function in high cervical level (C1-C4) spinal cord injury has been developed. It includes 24 channels of stimulation for shoulder, arm and hand muscles. By targeting the entire upper limb, which is completely paralyzed in this population, it is the most ambitious upper extremity FES system developed to date. Previous studies by Hoshimiya et al. used percutaneous electrodes to apply preprogrammed stimulation patterns to restore a very limited number of motions to $\mathrm{C} 4$ tetraplegics (Hoshimiya et al. 1989; Kameyama 1999). The implanted system aims to give the user a wide repertoire of movements, and to achieve this, a controller design more complex than a set of pre-defined stimulation patterns is needed.

The calculation of appropriate muscle stimulation levels for shoulder and arm movements is a difficult task. Force production by the muscles is time-varying and nonlinear, and in addition, muscles often cross multiple joints. They are also wide, and wrap around bones, and thus do not have straight lines of action. Specifically for muscles of the upper extremity, their role is not only to move the arm through the desired trajectory, but also ensure that the glenohumeral joint remains stable during the motion. No FES controllers for the upper extremity that take shoulder stability into account have yet been developed. For the successful implementation of a high tetraplegia FES system, this is a necessary step. 


\section{Summary of research}

A control design for a high level upper extremity neuroprosthesis is the focus of this study. The controller consists of a feedforward part, implemented using artificial neural networks, which is trained to approximate the inverse dynamics of the arm, including the relationships among muscle stimulation levels necessary for shoulder stability. The feedback part of the controller is a non-linear PD controller that adjusts the stimulation levels when there are errors in the arm movement due to external forces or muscle fatigue. Finally, the glenohumeral stability controller monitors the feedforward and feedback outputs and increases the stimulation of the shoulder stabilizing muscles according to the feedback contribution, which suggests there is an instability risk. The controller was developed and tested extensively in simulation, using both forward and inverse dynamic arm models. Subsequently, it was implemented in one subject with a high level tetraplegia FES system, and an adaptation method was developed to adjust the controller parameters to the characteristics of the specific individual.

In chapter 2, a musculoskeletal model of the upper extremity is presented, and evaluated with data collected from movements of able-bodied subjects. In chapter 3, this model is used to determine the optimal set of stimulating electrodes for the high tetraplegia neuroprosthesis, taking practical constraints, such as the hardware available, into account. The controller design, tested in a simplified model of the upper extremity with two degrees of freedom and six muscles, is presented in chapter 4. It is an evaluation of the trajectory tracking ability of the controller, and does not refer to shoulder stability, since the model does not include a complete glenohumeral joint. This is the subject of chapter 5, which presents the same design, augmented by a shoulder stability controller, applied to a larger 
scale arm model with five degrees of freedom and 22 muscles. Adaptation of the modelbased controllers for human implementation is first discussed in chapter 6. It describes an adaptation method for redundant systems, demonstrated first in a model of the elbow controlled by a pair of antagonistic muscles, and then in the elbow of the actual FES subject. Chapter 7 expands the adaptation technique to include the shoulder as well as the elbow, and presents the results of the controller design when applied to the shoulder and elbow of the FES user. Chapter 8 includes a discussion on the topics of this study and offers suggestions for future directions in the design of neuroprosthetic controllers. 


\title{
Chapter 2: A musculoskeletal model of the upper extremity for use in the development of neuroprosthetic systems
}

\begin{abstract}
Upper extremity neuroprostheses use functional electrical stimulation (FES) to restore arm motor function to individuals with cervical level spinal cord injury. For the design and testing of these systems, a biomechanical model of the shoulder and elbow has been developed, to be used as a substitute for the human arm. It can be used to design and evaluate specific implementations of FES systems, as well as FES controllers. The model can be customized to simulate a variety of pathological conditions. For example, by adjusting the maximum force the muscles can produce, the model can be used to simulate an individual with tetraplegia and to explore the effects of FES of different muscle sets. The model comprises six bones, five joints, nine degrees of freedom, and 29 shoulder and arm muscles. It was developed using commercial, graphics-based modeling and simulation packages that are easily accessible to other researchers and can be readily interfaced to other analysis packages. It can be used for both forward-dynamic (inputs: muscle activation and external load; outputs:motions) and inverse-dynamic (inputs: motions and external load; outputs: muscle activation) simulations. Our model was verified by comparing the model-calculated muscle activations to electromyographic signals recorded from shoulder and arm muscles of five subjects. As an example of its application to neuroprosthesis design, the model was used to demonstrate the importance of rotator cuff muscle stimulation when aiming to restore humeral elevation. It is concluded that this model is a useful tool in the development and implementation of upper extremity neuroprosthetic systems.
\end{abstract}




\section{Introduction}

Neuroprosthetic systems restore function after spinal cord injury (SCI) using a combination of functional electrical stimulation (FES) and reconstructive surgeries such as tendon transfers (Keith and Lacey 1991). An FES system consists of a controller that outputs the muscle excitations needed for a particular task and electrodes that deliver the stimulation to the appropriate paralyzed muscles. Upper extremity neuroprostheses to date have focused on restoring hand function in individuals with mid to low cervical level injuries (C5-C8), using open loop controllers that are tuned and tested using trial and error (Keith et al. 1988; Kilgore et al. 1989). However, purely experimental methods are inefficient in cases of high cervical level injuries (C1-C4), where almost all voluntary muscle function below the neck is lost. In these cases, there are a large number of shoulder and elbow muscles that must be controlled by the neuroprostheses, many of which generate moments about two or more degrees of freedom. For example, the biceps muscle originates on the scapula and inserts on the radius, which means it crosses the glenohumeral, humero-ulnar, and radio-ulnar joints. As a result, the task of determining which muscles to stimulate and developing appropriate control schemes becomes more complicated. Trial and error techniques would require extensive testing of all possible muscle combinations, a time-consuming process for both the researcher and the FES participant. The use of a musculoskeletal model can facilitate the design and testing of the system, minimizing the inconvenience to the subjects.

There have been a number of computer models of the shoulder developed in the last few decades, and most of them are strictly inverse. The first models were two-dimensional, restricted to single-motion patterns, and did not include all the shoulder muscles (DeLuca and Forrest 1973; Dul 1987). There are very few three-dimensional models, mostly because of 
the complexity of the shoulder mechanism. The "Swedish" shoulder model was developed by Karlsson and Peterson, based on morphological measurements by Hogfors et al. (Karlsson and Peterson 1992; Hogfors et al. 1987). The "Delft" model, by van der Helm, is based on morphological data from Veeger et al. (van der Helm 1994; Veeger et al. 1991, 1997). The "Newcastle" shoulder model, described by Charlton and Johnson, is based on data from Johnson et al., van der Helm and Veeger (Charlton and Johnson 2000). The only forward model developed to date, by van der Helm and Chadwick, is still under development and has very slow simulation speed (van der Helm and Chadwick 2002).

In this study, a new model of the shoulder and elbow was developed that can be used for both inverse and forward dynamic simulations, thus providing a complete description of the upper extremity. Verification of the model was achieved in an inverse dynamic manner, by correlating the EMG activity in different muscles during arm movements performed by able-bodied individuals, to the activation patterns predicted by inverse dynamic simulations using the model. Finally, an example of the model use in neuroprosthesis design is presented. The model was adjusted to simulate a C3 SCI individual, and the effect of two different sets of stimulated muscles in the performance of a humeral abduction movement was investigated.

\section{Methods}

\section{Model Construction}

The muscle and joint parameters for the model were obtained from cadaver studies by Klein-Breteler et al. (1999). These parameters include the position of joint centers, inertial parameters for body segments, the number of elements representing each muscle, and the 
optimal fiber length, origin and insertion, tendon slack length, physiological cross-sectional area and pennation angle of every element.

The software used to create the model was SIMM (Musculographics, Inc.), a graphics-based system developed especially for musculoskeletal modeling. The model consists of six bones (sternum, clavicle, scapula, humerus, ulna and radius) and five joints (sterno-clavicular, acromio-clavicular, glenohumeral, humero-ulnar and radio-ulnar). The three joints at the shoulder are ball joints, with three degrees of freedom each, while the elbow and forearm joints are one-degree-of-freedom, single-axis joints. The model includes the so-called scapulothoracic gliding plane (van der Helm 1994), which means that the medial border of the scapula is constrained to maintain contact with the thorax.

The muscles are represented by one or more elements, depending on their size and the width of their attachment site (Table 2-1). The model includes 29 muscles, with a total of 138 elements. If the muscle wraps over a bone surface, a "wrap object" is used to represent that surface such that the muscle path is not allowed to pass through the bone.

\begin{tabular}{lr|lr}
\hline Muscle & $\begin{array}{r}\text { Number of } \\
\text { elements }\end{array}$ & Muscle & $\begin{array}{r}\text { Number of } \\
\text { elements }\end{array}$ \\
\hline Trapezius, scapular part (mid and lower) & 11 & Biceps, long head & 1 \\
Trapezius, clavivular part (upper) & 2 & Biceps, short head & 2 \\
Levator scapulae & 2 & Triceps, long head & 4 \\
Pectoralis minor & 4 & Triceps, medial head & 5 \\
Rhomboid & 5 & Triceps, lateral head & 5 \\
Serratus anterior & 12 & Latissimus dorsi & 6 \\
Deltoid, scapular part (post and mid) & 11 & Pectoralis major, thoracic part & 6 \\
Deltoid, clavicular part (anterior) & 4 & Pectoralis major, clavicular part & 2 \\
Coracobrachialis & 3 & Brachialis & 7 \\
Infraspinatus & 6 & Brachioradialis & 3 \\
Teres minor & 3 & Pronator teres & 2 \\
Teres major & 4 & Supinator & 5 \\
Supraspinatus & 4 & Pronator quadratus & 3 \\
Subscapularis & 11 & Anconeus & 5 \\
\hline
\end{tabular}

Table 2-1: The muscles included in the model, and the number of elements that represent each one. 
In order to run dynamic simulations, two additional software packages were used. The first package was SD/FAST (Parametric Technology Corp), which uses parameter files created by SIMM to compute the equations of motion of the modeled system. The second package, the Dynamics Pipeline (Musculographics, Inc.), connects SIMM to SD/FAST in order to perform forward and inverse dynamic simulations. In the case of forward dynamic simulations, the model inputs are muscle activations and possible external loads, and the output is the resulting movement. In the case of inverse dynamic simulations, the desired movement and possible external loads are the inputs, and the required muscle activations are the outputs.

In the second case, since there are more muscles than degrees of freedom in the musculoskeletal system, a non-linear constrained optimization routine, CFSQP (AEM Design) is used in order to solve the load-sharing problem (Tsirakos et al. 1997). Three types of constraints are included: first, for every degree of freedom, the muscle forces are constrained to produce the torques required by the input movement. Second, the muscle forces acting on the glenohumeral joint are constrained to pull the humerus towards the glenoid cavity, thus ensuring the joint stability. And third, the muscle forces acting on the scapula are constrained to keep the scapula pressed against the thorax, in accordance with the scapulothoracic gliding plane, described earlier. The objective function currently used was proposed by Praagman et al. (2006) to minimize energy consumption:

$$
\min \sum E_{m}=\sum\left(E_{f}+E_{a}\right)=\sum m\left\{c_{1} \frac{F_{m}}{P C S A}+c_{2}\left(\frac{F_{m}}{F_{\max }}\right)^{2}\right\}
$$

where $E_{f}$ and $E_{a}$ are the muscle energy consumption due to the detachment of cross bridges and re-uptake of calcium respectively, $m$ is the muscle mass, $F_{m}$ is the muscle force, PCSA is 
the physiological cross-sectional area, $F_{\max }$ is the maximum muscle force, and $c_{1}$ and $c_{2}$ are two constants chosen to achieve equal contributions from the linear and nonlinear terms at $50 \%$ activation (Praagman et al. 2006).

\section{Experimental setup}

Five able-bodied subjects participated in the study after giving informed consent. The subjects were asked to perform a set of movements with their right arm, while their arm position and the EMG signals of selected arm muscles were recorded. Figure 2-1 shows the experimental setup.

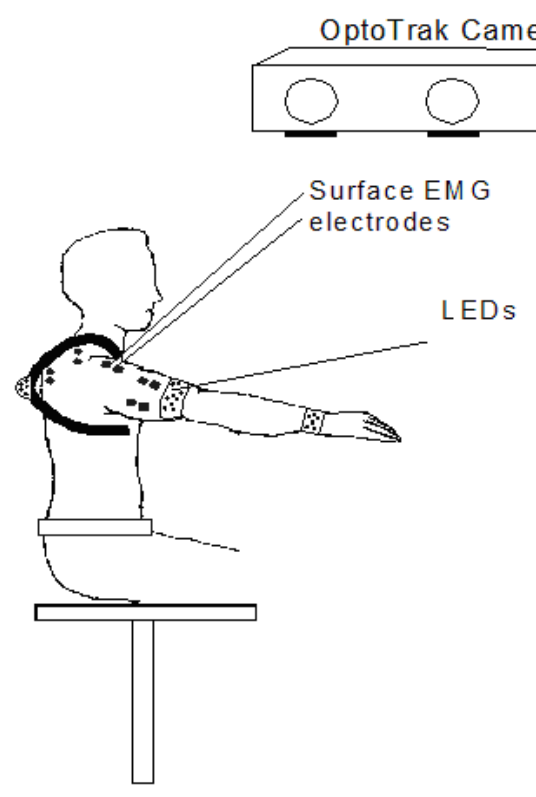

Figure 2-1: Experimental Setup. Sets of LED were fixed over the thorax, humerus and forearm of the subjects, and the threedimensional position of these segments was recorded using three cameras. The positions of the clavicle and scapula were estimated using a regression model based on the scapulohumeral rhythm. Surface and percutaneous electrodes measured the EMG signals from selected shoulder and arm muscles. 
The arm movements were recorded using an Optotrak system (Northern Digital Inc.) that consists of three cameras capable of recording the 3D positions of light emitting diodes (LED) located within the workspace. Sets of LED were fixed over the thorax, humerus and forearm of the subjects to locate the three-dimensional position of these segments. The locations of the scapula and clavicle are difficult to track dynamically using markers fixed to the skin, because they show large displacements with respect to the skin. However, there is a well-defined relation between scapular and humeral motion, which is that during a normal arm elevation movement, about one-third is due to scapulothoracic motion, and two-thirds is due to glenohumeral rotation (De Groot and Brand 2001). Moreover, because of the closed chain between the thorax, clavicle and scapula created by the sternoclavicular and acromioclavicular joints, and the scapulothoracic gliding plane, the orientations of the clavicle and scapula are related. Based on these relations, a three-dimensional regression model was built, using the positions of scapular, clavicular, and humeral bony landmarks recorded in a series of static trials covering the workspace. The bony landmarks were chosen according to van der Helm, 1997, and the recorded positions consisted of five levels of humeral elevation between $0^{\circ}$ and $120^{\circ}$, evenly distributed in that range, in the coronal (i.e. elevation to the side), scapular ( $45^{\circ}$ from coronal) and sagittal planes (to the front), with the elbow fully extended. During the dynamic trials, the regression model was used along with the orientation of the humerus to determine the dynamic orientation of the scapula and the clavicle.

EMG data were recorded in the first three subjects using surface electrodes on six muscles. In order to evaluate the model predictions for more muscles, including ones too deep to be easily accessible by surface electrodes, the last two subjects had surface electrodes 
placed on eight muscles, and fine wire percutaneous electrodes placed on three muscles: infraspinatus, supraspinatus and pronator teres. The list of muscles for each subject is shown in table 2-2. All EMG signals were rectified, low pass filtered at a cutoff frequency of $4 \mathrm{~Hz}$, and normalized by their values during maximum voluntary contractions.

\begin{tabular}{l|r|r|r}
\hline subjects & S1 and S2 & S3 & S4 and S5 \\
\hline surface & biceps & biceps & biceps \\
electrodes & triceps & triceps & triceps \\
& anterior deltoid & anterior deltoid & anterior deltoid \\
& middle deltoid & & posterior deltoid \\
posterior deltoid & posterior deltoid \\
& upper trapezius & upper trapezius & $\begin{array}{r}\text { uppezius } \\
\text { pectoralis major } \\
\end{array}$ \\
& & & $\begin{array}{r}\text { latissimus dorsi } \\
\text { serratus anterior }\end{array}$ \\
& & & infraspinatus \\
& & & supraspinatus \\
percutaneous & & & pronator teres \\
electrodes & & & \\
\hline
\end{tabular}

Table 2-2: Electrodes used to measure EMG signals for correlation to model-predicted activations. In the first three subjects, only surface electrodes were used, placed on six muscles. In the last two subjects, eight surface and three fine wire percutaneous electrodes were used.

The same movements were performed by all subjects. They were relatively slow (joint velocities around 30 degrees/second), in order to simulate the expected performance of a person with an FES system. They consisted of a set of planar movements: shoulder abduction/adduction in the coronal, scapular, and sagittal planes, horizontal flexion/extension, internal/external rotation, elbow flexion/extension and forearm pronation/supination, reaching movements to three different heights: knee level, shoulder level, and above shoulder level, and two movements simulating activities of daily living (ADL): eating, and combing the hair. Data were recorded at $30 \mathrm{~Hz}$. 


\section{Model evaluation}

The model predictions were evaluated based on the shape and timing of the signals and not the actual amplitude, because EMG amplitude depends on the properties and locations of the electrodes used and therefore is not a reliable measure of muscle activation. For this reason, the model was evaluated by cross-correlation of the model-predicted activations and the EMG signals from the selected muscles.

In order to avoid correlating signals with very low amplitudes but (in the case of EMG) with noisy baseline activity, muscles with EMG values that did not rise above a specified threshold for the duration of a given trial were excluded from the correlation calculations for that trial. The threshold was defined as one standard deviation above the baseline, as described in Hodges and Bui 1996. For these cases, a different measure was used to summarize the ability of the model to predict the lack of activity in a given muscle: the false positive rate. That is, the number of trials in which muscle activity was predicted by the model but none was measured using EMG was expressed as a percentage of the total number of trials where no activity was measured using EMG.

\section{Application to C3 SCI with and without FES}

A spinal cord injury at the $\mathrm{C} 3$ level causes paralysis of almost all upper extremity muscles, with the possible exception of the levator scapulae and upper trapezius (Kirsch et al. 2001). To simulate a subject with C3 SCI, all other muscles were set to have zero active force, and in addition, the maximum force for the levator scapulae and upper trapezius was set to $50 \%$ of able-bodied maximum force to simulate the effects of possible muscle 
denervation and partial paralysis (within the range of $20-60 \%$ measured by Kobetic and Marsolais 1994).

Two sets of muscle candidates for FES were evaluated: the first included the serratus anterior and deltoids, and the second included the serratus anterior, deltoids and three rotator cuff muscles (supraspinatus, infraspinatus and subscapularis). To simulate FES, the maximum forces of these sets of muscles were set to $50 \%$ of the corresponding able-bodied maximum muscle forces. This reduction simulates possible muscle denervation, disuse atrophy, and limited spatial muscle activation due to electrode placement.

The task used to evaluate the effect of FES was abduction of the arm in the coronal plane from 0 to 100 degrees of elevation, with the elbow fully extended. Inverse dynamic simulations were performed by the model under four conditions: (1) able-bodied, (2) C3 SCI, (3) C3 SCI with FES of muscle set 1, and (4) C3 SCI with FES of muscle set 2. The maximum humeral elevation for each case was determined either by the successful completion of the movement (i.e. the model reached 100 degrees of elevation), or by the highest elevation achieved before the simulation failed. In the case of failure, the model specified which constraint was violated: the muscle forces were either insufficient for the required joint torques, they caused instability of the glenohumeral joint, or they were unable to keep the scapula pressed against the thorax.

\section{Results}

Figure 2-2 demonstrates the evaluation procedure, using as an example, the middle deltoid of subject 2 during a humeral abduction movement. It shows the raw EMG data (part A), the processed EMG data (part B), the corresponding model-predicted middle deltoid 
activation (part C), and a comparison of the processed EMG and model-predicted activation (part D). In part D, it is shown that the timing of the measured EMG and the model-predicted activation are quite similar. Also note that in part $\mathrm{D}$, the magnitudes of the signals are not the same, but this is not taken into account since the model verification is based on timing, not amplitude. The cross-correlation in this case was 0.850 .
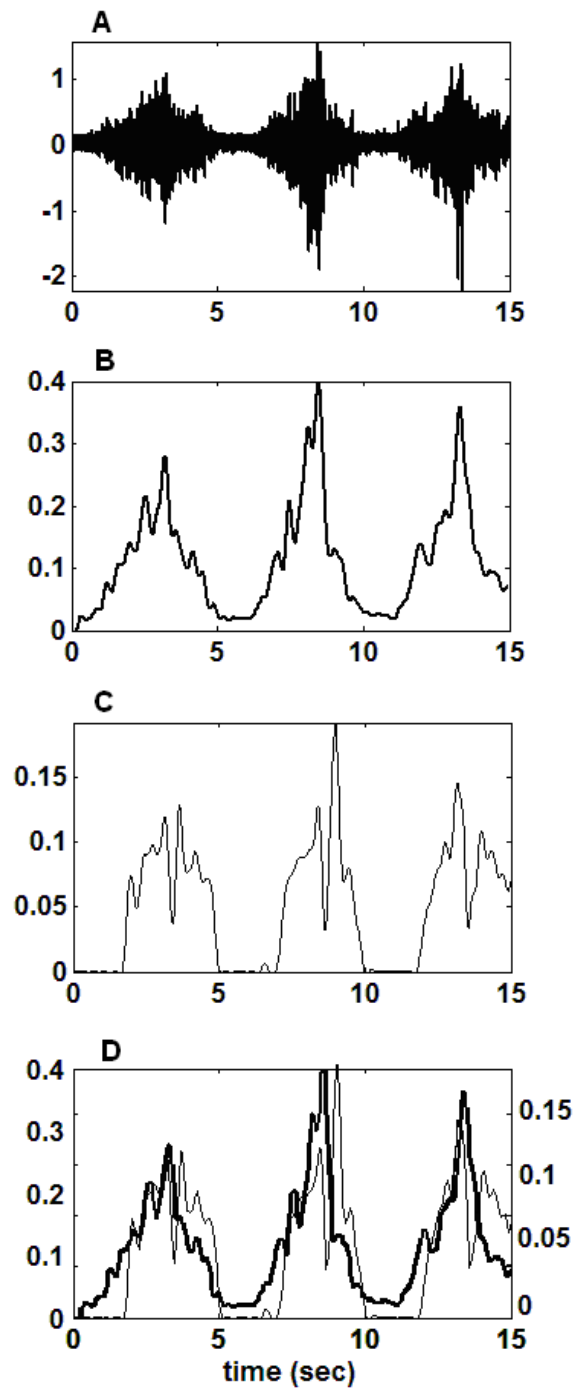

Figure 2-2: EMG recording, processing, and comparison to model-predicted activations. For example, the EMG and model-predicted activations of middle deltoid muscle during a repeated humeral abduction movement are illustrated. Raw EMG (A), processed (rectified, low pass filtered at $4 \mathrm{~Hz}$ and normalized) EMG (B), model-predicted activation (C), processed EMG (black line) and model-predicted activation (grey line) (D). For part $\mathrm{D}$, the vertical axis for the EMG is on the left, and for the activation is on the right. 
Figure 2-3 shows an example of a typical correlation between EMG and modelpredicted activation. This is also a humeral abduction movement, and the muscles shown are the serratus anterior (A), supraspinatus (B) and infraspinatus (C). These plots show that the model-predicted activation peaks generally match the EMG peaks. The similarity of shape is reflected in the cross-correlation values, which in this case were $0.610,0.650$ and 0.625 respectively.
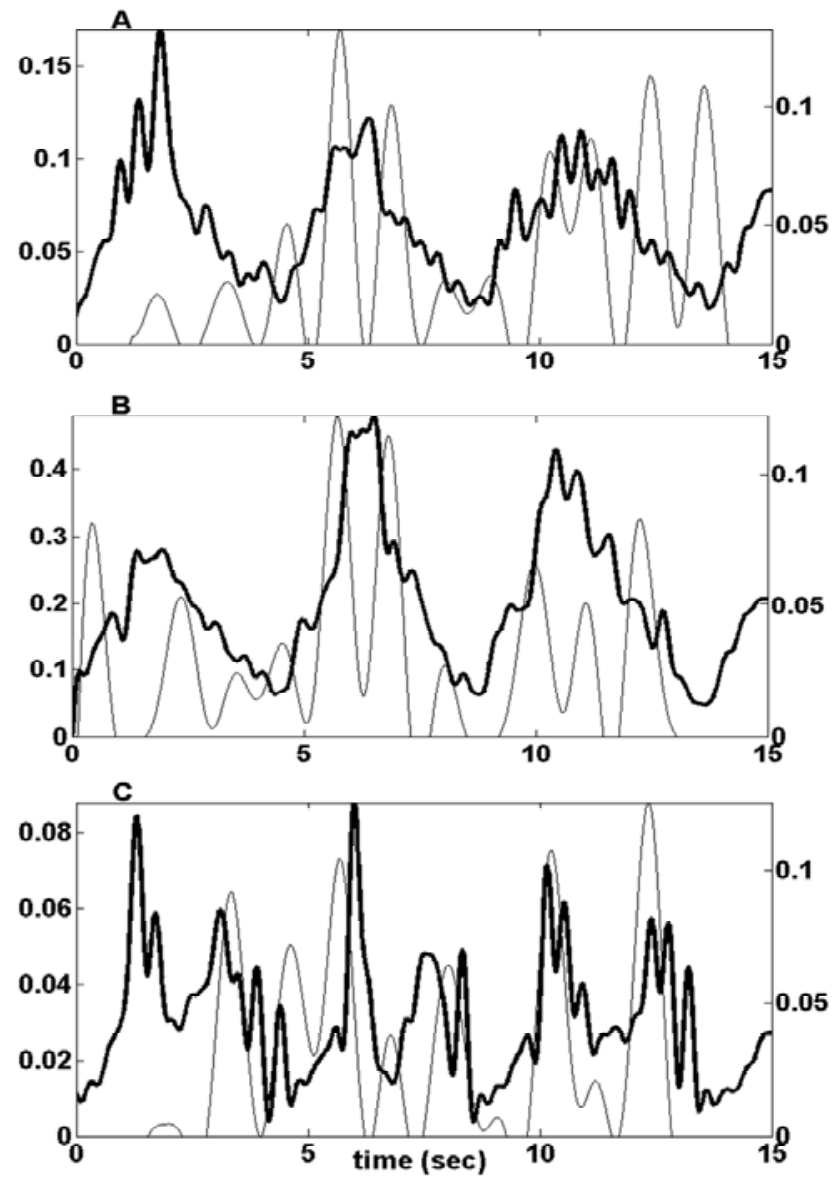

Figure 2-3: Serratus anterior (A), supraspinatus (B), and infraspinatus (C) signals during a repeated humeral elevation movement. For all muscles, the processed EMG signal is the black line, and the model-predicted activation is the grey line. The vertical axes for the EMG signals are on the left, and for the model-predicted activations are on the right. 
Figure 2-4 shows a situation where the correlation between EMG signals and model predictions is less accurate, for the biceps (A) and triceps (B) during a repeated elbow flexion/extension movement. In this figure, the amplitudes were not scaled, to highlight the model-predicted inactivity of the triceps, which does not agree with the active EMG measurement. The cross-correlation values for the biceps and triceps were 0.391 and 0.290 respectively.

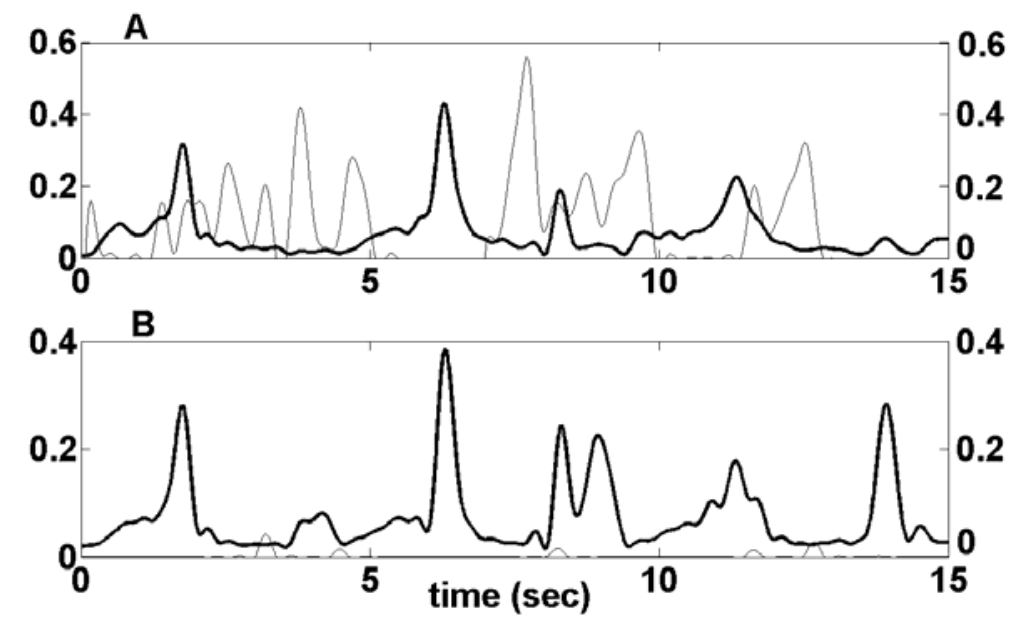

Figure 2-4: Biceps (A) and triceps (B) signals during a repeated elbow flexion/extension movement. The processed EMG signal is the black line, and the model-predicted activation is the grey line. Cocontraction of the biceps and triceps is recorded in the EMG signals, but is not predicted by the model.

Figure 2-5 shows the cross-correlation values between activations and EMG signals for the measured muscles of each of the five subjects, averaged across all tasks. Averaged across subjects, the cross-correlation ranged from 0.282 for the triceps to 0.726 for the middle deltoid. The overall mean correlation was 0.455 . In most subjects, the three heads of the deltoid had the best predictions, and the biceps and triceps had the worst. For the last two subjects that had more EMG electrodes, the prediction was poor for the latissimus dorsi, but 
generally good for the muscles that stabilize the scapula and the humerus: serratus anterior, supraspinatus and infraspinatus.

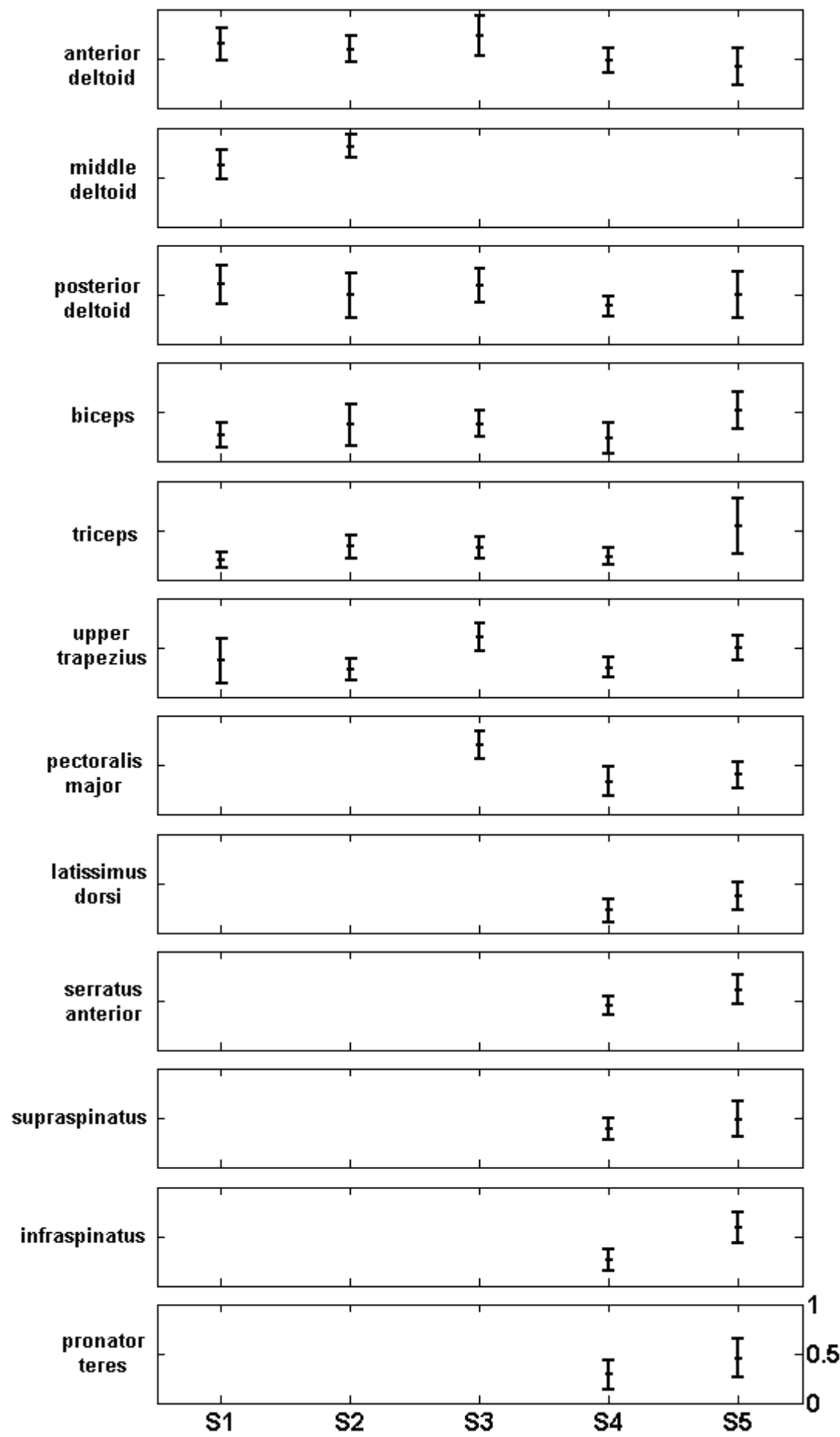

Figure 2-5: Cross-correlation between EMG and model-predicted muscle activations, averaged across tasks. Every row corresponds to one muscle, and every column to one subject. The vertical scale of every plot ranges from 0 to 1 . 
The false positive rate is presented in Table $2-3$. The lowest value was $23.3 \%$ for the triceps, while the highest was $80 \%$ for the middle deltoid, but it should be noted that this was calculated from only five trials, since the middle deltoid showed above-threshold EMG activity in all but five trials. Overall, no muscle activation was systematically overestimated by the model.

\begin{tabular}{l|rrr}
\hline & $\begin{array}{r}\text { EMG off } \\
\text { act. on }\end{array}$ & $\begin{array}{r}\text { Total } \\
\text { EMG off }\end{array}$ & $\begin{array}{r}\text { False positive rate } \\
\text { (\%) }\end{array}$ \\
\hline anterior deltoid & 5 & 13 & 38.5 \\
middle deltoid & 4 & 5 & 80.0 \\
posterior deltoid & 20 & 33 & 60.6 \\
biceps & 7 & 25 & 28.0 \\
triceps & 10 & 43 & 23.3 \\
upper trapezius & 14 & 30 & 46.7 \\
pectoralis major & 17 & 48 & 35.4 \\
latissimus dorsi & 9 & 34 & 26.5 \\
serratus anterior & 6 & 15 & 40.0 \\
supraspinatus & 4 & 14 & 28.6 \\
infraspinatus & 11 & 41 & 26.8 \\
pronator teres & 8 & 25 & 32.0 \\
\hline Total & 115 & 326 & 35.3 \\
\hline
\end{tabular}

Table 2-3: False positive rate. The first column is the number of trials in which muscle activity was predicted by the model but none was measured using EMG. The second column is the total number of trials where no activity was measured using EMG. The third column is the false positive rate, defined as the ratio of the first two columns.

Figure 2-6 shows the results of inverse dynamic simulations performed by the model adjusted to represent an able-bodied subject, a C3 SCI individual without FES, and a C3 SCI individual with FES of two different muscle sets. The maximum humeral elevation possible by the able-bodied simulation was 100 degrees. Without FES, the model failed immediately 
(i.e., zero elevation) due to the extended paralysis at this level of injury. Simulation of FES of the serratus anterior and deltoids produced humeral elevation of 12 degrees. For higher elevations, the model failed because glenohumeral stability was not maintained. Addition of the rotator cuff muscles to the simulation increased the maximum elevation to 81 degrees (i.e., almost to the able-bodied range).

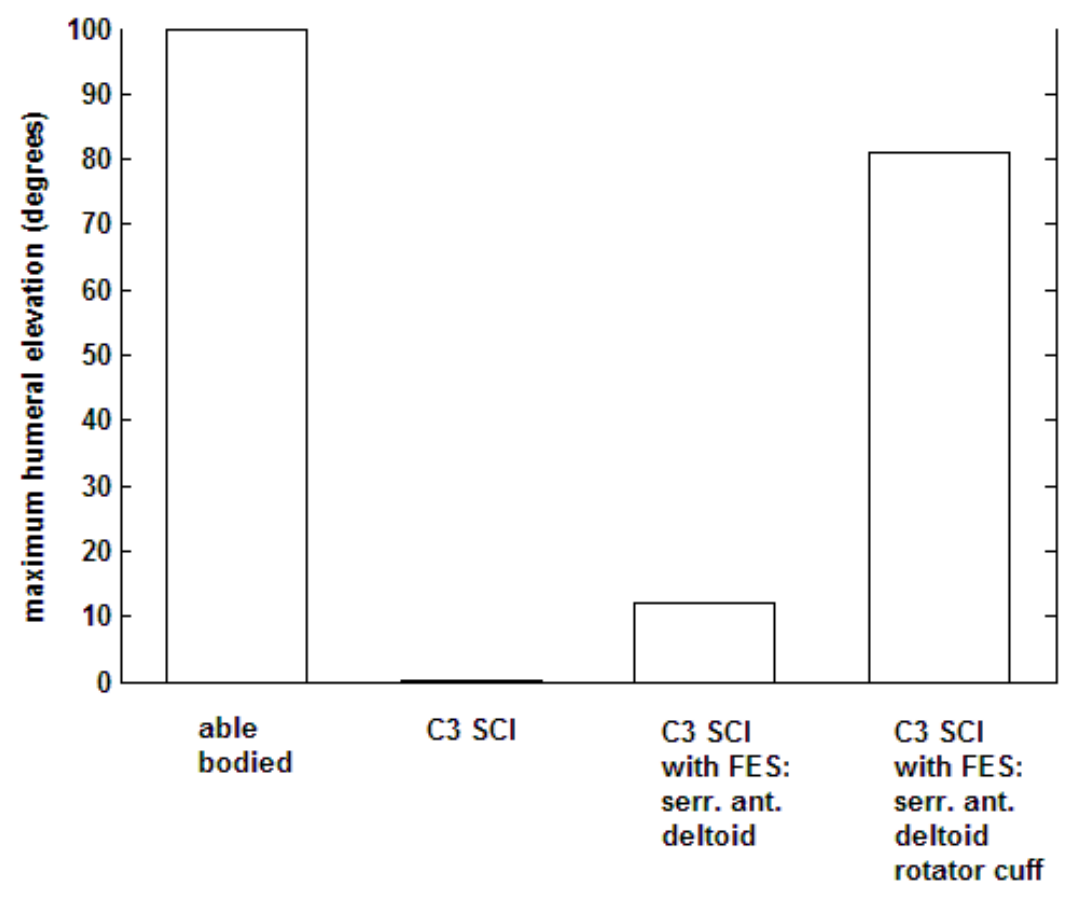

Figure 2-6: Maximum humeral elevation achieved in four cases: (a) able-bodied (b) C3 SCI (c) C3 SCI with FES of serratus anterior and deltoids (d) C3 SCI with FES of serratus anterior, deltoids, infraspinatus, supraspinatus and subscapularis.

\section{Discussion}

A dynamic model of the upper extremity has been described. It was developed using a set of commercially available software packages, with a user-friendly, graphical interface. It can be used both for forward and inverse dynamic simulations. For the three heads of the 
deltoid, which are primary arm movers, the model predicts activations that correspond to the timing of the measured EMGs quite accurately. The same can be said for the serratus anterior and the rotator cuff muscles (infraspinatus and supraspinatus), whose role is to stabilize the scapula and the glenohumeral joint respectively. Our model can therefore accurately predict the functions of key muscles of the upper extremity, making it a valuable tool for exploratory simulations aiming to provide insight into the performance of the real arm under various conditions. Specifically, this model can be a useful tool for the design and testing of upper extremity neuroprosthetic systems.

Validation of musculoskeletal models is generally difficult to achieve. Thorough validation is not practical because the muscle excitation patterns that are the model inputs are not directly measurable in the actual musculoskeletal system. For his model of the upper extremity, van der Helm compared the predicted muscle forces with electromyographical (EMG) recordings (van der Helm 1994). The problem with this approach is that it did not take into account the dependency of the EMG amplitude on muscle length. van der Helm concluded that EMG amplitude cannot be used to validate musculoskeletal models, and he pointed out that in this case, only verification, and not strict validation of the model is possible. Instead of EMG, Praagman et al. (2003) suggested the use of local oxygen consumption in a muscle, measured by near infrared spectroscopy (NIRS). However, the dynamic properties cannot be evaluated with this method since NIRS cannot be used for dynamic measurements. In other studies, evaluation of the model is performed by comparing predicted forces with measurements from the literature. This method cannot give quantitative conclusions, since the modeling parameters are different among the studies, but it can result in a qualitative agreement (Karlsson and Peterson 1992; Charlton and Johnson 2000). 
In this study, the performance of the model was verified by comparing the shape over time of the calculated muscle activations to the corresponding EMG recordings. Figure 2-3 shows that the best correlation is achieved for the three heads of the deltoid, while for muscles like the biceps and triceps, the agreement is not good. One reason for this might be co-contraction of the biceps and triceps during the low velocity movements examined, something that would not be predicted by the model using the selected energy-related cost function for load sharing. Figure 2-4 shows an example of this. The EMG signals for the biceps and triceps reveal significant co-contraction during flexion, as opposed to the model that does not predict any triceps activity, most likely because in this task, extension can be achieved entirely through gravity. Since the nervous system probably uses different strategies for different conditions, a cost function that takes into account the stiffness of the arm could work better for this movement. However, an FES system does not aim to replace the nervous system, so the choice of cost function, even if not a perfect model of an intact nervous system, can still lead to muscle activation patterns that, applied by the FES system, will produce the desired movement.

In some cases, disagreement between EMG and model-predicted activation can probably be attributed to EMG measurement errors. The latissimus dorsi has a small crosscorrelation value $(0.310$, averaged across subjects) and it would not be expected to be active unless the subject pushes down or adducts against resistance (Jenkins 2002). Since neither of these actions were included in the task set, it is likely that the surface electrodes detected EMG signals from neighboring muscles such as the serratus anterior. In fact, a crosscorrelation analysis of the signals from latissimus dorsi and serratus anterior produces an average value of 0.886 . This could be due to poorly placed electrodes, or (more likely) to 
inter-muscle crosstalk, a common problem when surface electromyography is used (Solomonow et al. 1994).

Another possible reason for disagreement between EMG and model-predicted activation could be that the cadaver-based model parameters do not correspond well to the participants of this study. Parameters like the maximum muscle force cannot be directly measured in individual subjects. However, it is unlikely that such differences will significantly impact the effectiveness of model-based simulations in reaching general conclusions about the configuration of potential FES systems. In the example of humeral abduction presented above, the model predicted that including rotator cuff muscles in the stimulation set can greatly improve the restored function. Even though the exact level of improvement may be different depending on the specific person's remaining function and possible denervation, the model results can be used as a guideline for choosing the most effective muscle set for FES.

In conclusion, we believe that the model presented here can significantly facilitate the design of neuroprosthetic systems, by replacing the current trial-and-error methods that would be impractical in the case of high level injuries with a simulation system that can explore the mechanical considerations associated with different movements and different stimulated muscle sets. The model was successfully verified using EMG as a measure of muscle activation and is now ready for use in aiding the development of advanced FES systems for restoring arm and shoulder movements. 


\title{
Chapter 3: Model-based selection of muscle and nerve cuff electrodes for a high tetraplegia neuroprosthesis
}

\begin{abstract}
A neuroprosthetic system based on functional electrical stimulation (FES) is under development to restore shoulder and arm function to individuals with complete paralysis due to high level spinal cord injury (SCI). To determine the optimal set of muscle and nerve cuff electrodes to be included in the neuroprosthesis, a musculoskeletal model of the upper extremity was used in the place of the human arm. The candidate sets for stimulation were created based on the muscles that are available for stimulation at this level of injury and the number and type of electrodes available in the proposed system. Using each of the candidate electrode sets, a large number of inverse dynamic model simulations were performed to determine if a variety of key functional movements could be accomplished by a given set. The set that allowed the most movements to be performed was chosen as the optimal set for stimulation. This set includes two selective nerve cuff electrodes on the radial and musculocutaneous nerves, four non-selective cuff electrodes on the axillary, suprascapular, upper subscapular and long thoracic nerves, and five muscle electrodes on the upper and lower pectoralis major, rhomboids, pronator quadratus and supinator. The model-based optimal set will be the guide for determining the target set of the high tetraplegia neuroprosthesis.
\end{abstract}




\section{Introduction}

Neuroprostheses restore motor function after spinal cord injury (SCI) by applying functional electrical stimulation (FES) to paralyzed muscles. The number of muscles targeted by the FES system is a function not only of the level of injury, which determines the extent of the paralysis, but also of the number of stimulation channels available from the neuroprosthesis itself. For example, arm function in low tetraplegia (C7 SCI) can be significantly improved with stimulation of just the triceps (Crago et al. 1998), since most of the other arm muscles are under voluntary control. However, in the same population, all hand muscles are typically paralyzed, and FES systems that restore basic hand function (i.e. grasp and release) use at least 8 stimulating channels (Keith et al. 1998). Restoring more natural and dexterous hand function would require many more channels than the current neuroprostheses can provide (Kilgore et al. 1989). Our study focuses on restoration of shoulder and arm function to individuals with high tetraplegia (C1-C4 SCI), whose upper extremities are almost entirely paralyzed, so it is the available stimulating channels, and not the remaining motor function, that limits the number of muscles targeted for FES.

As the number of available stimulating channels increases, the problem of determining which muscles to stimulate to restore the most function to a specific individual becomes both difficult and important for the success of the FES system. One way to solve this problem is to identify the appropriate muscle groups based on their known functions, and choose a subset of these according to their surgical accessibility and level of response to electrical stimulation. In the case of the hand grasp system mentioned above, a limited group of primary muscles for hand function was chosen, and supramaximal surface stimulation was used in order to exclude muscles with complete denervation (Kilgore et al. 1989). Similarly, 
in the design of lower extremity FES systems for standing, the target muscles were chosen based on biomechanical analysis, feedback from other users, and prior testing with surface and percutaneous electrodes (Davis et al. 2001). These methods work well in systems that restore a limited number of functions, such as simple hand grasp or a fixed standing pattern. However, in the case of high tetraplegia, we would like to restore many different movements (e.g., reaching to the front or the side and eating) and different sets of muscles are important in each case. Moreover, many muscles do not have obvious effects on the movement of limb segments, but rather act to stabilize joints (e.g. the rotator cuff muscles stabilize the glenohumeral joint). Finally, feedback from previous users is limited, since earlier FES approaches to individuals with high tetraplegia were more than 15 years ago and were based on temporary surface (Nathan 1989; Nathan and Ohry 1990) or percutaneous (Hoshimiya et al. 1989) stimulation. Furthermore, the stimulation patterns were based on able-bodied EMG patterns rather than the biomechanical requirements on muscles with partial denervation and disuse atrophy, and relied upon external support of the weight of the arm. For these reasons, the optimal muscle set for stimulation in high tetraplegia cannot be identified by using only previous experience and biomechanical observations.

We have instead adopted an approach that uses a musculoskeletal model in place of the actual human arm to investigate the effect of different stimulating electrode sets on the shoulder and arm function. Heilman et al. used a musculoskeletal model of the human lower extremities to determine the optimal set of additional muscles that should be stimulated by a 16-channel neuroprosthesis, as opposed to the existing 8-channel system, to improve balance and allow the user to shift postures (Heilman et al. 2006). As a first step towards modelguided muscle selection for upper extremity FES, an upper extremity musculoskeletal model 
was used to determine the minimum muscle set for an FES system to restore function to high level tetraplegia (Acosta et al.). That study focused on maintaining static postures in different locations (arm elevation from resting to horizontal in the coronal and scapular planes, and hand locations from a tabletop to the mouth), rather than restoring actual dynamic movements.

In this project, a musculoskeletal model reflecting the mechanics of an individual with high tetraplegia was used in simulation to determine the optimal electrode set for an FES system capable of restoring a variety of simple but functionally important movements. The model adjustments accounted for the limited remaining voluntary function, the reduced force capacities in the paralyzed but stimulated muscles, and the number of stimulation channels available (far less than the number of paralyzed muscles). Furthermore, the effects of the two different types of electrodes available for an actual neuroprosthesis were simulated. Muscle based electrodes are placed on the surface of a muscle (Kilgore et al. 2003) or inserted into a muscle (Memberg et al. 1994) and activate nerve branches in the immediate vicinity of the electrode. They are most effective for smaller (e.g. hand) or physically isolated (e.g. pectoralis major) muscles. Nerve cuff electrodes wrap around a peripheral nerve before they reach a muscle, and activate all the muscle fibers innervated by that nerve (Naples et al. 1998). This makes them particularly useful for shoulder muscles that are too broad to be activated by a single muscle electrode, such as the nine-compartment serratus anterior, for muscles that might sustain damage due to the electrode sliding against bones during movement (e.g. deltoid), or for muscles that are deep or otherwise represent difficult surgical targets (e.g. the rotator cuff muscles). In addition, if multiple muscles are innervated by the same nerve, (e.g. radial nerve), the nerve cuff may either activate all of 
them, or, if it has multiple contacts, selectively activate different muscles (Grill and Mortimer 2000). Finally, nerve cuff electrodes can be placed in different locations along the nerve. For example, a cuff electrode can be placed on the radial nerve distal to the branch innervating the long head of the triceps, and in this way avoid stimulating that muscle.

Using model simulations rather than trial and error experimentation allowed us to objectively evaluate the mechanical and functional impact of different electrode sets prior to human implementation. The results will provide the starting point for selecting muscle and nerve electrodes for the actual neuroprosthetic system.

\section{Methods}

The model used in this study was built in SIMM (Musculographics, Inc.), a graphicsbased system developed especially for musculoskeletal modeling. The model consists of six bones (sternum, clavicle, scapula, humerus, ulna and radius) and five joints (sternoclavicular, acromio-clavicular, glenohumeral, humero-ulnar and radio-ulnar). The three joints at the shoulder are ball joints, with three degrees of freedom each, while the elbow and forearm joints are one-degree-of-freedom, single-axis joints. The model includes the socalled scapulothoracic gliding plane (van der Helm 1994), which means that the medial border of the scapula is constrained to maintain contact with the thorax. The muscles are represented by one or more contractile elements, depending on their size and the width of their attachment site. The model includes 29 muscles, with a total of 138 contractile elements. The model parameters were obtained from cadaver studies by Klein-Breteler et al. (1999). These parameters include the position of joint centers, inertial parameters for body segments, the number of elements representing each muscle, and the optimal fiber length, 
origin and insertion, tendon slack length, physiological cross-sectional area and pennation angle of every element.

The model was customized to simulate a person with high level spinal cord injury. An injury at high cervical levels causes paralysis of almost all upper extremity muscles, with the usual exception of the levator scapulae and upper trapezius (Kirsch et al. 2001). To simulate a subject with high level SCI, all other muscles were set to have zero active force, and in addition, the maximum forces for the levator scapulae and upper trapezius were set to $50 \%$ of able-bodied maximum force, to simulate the effects of possible muscle denervation and partial paralysis.

For the muscles assumed to be included in the set of muscles activated by the neuroprosthetic system using muscle or nerve cuff electrodes, the maximum forces were fixed at $50 \%$ of the average able-bodied maximum forces. This reduction simulates possible muscle denervation, and in the case of muscle electrodes, limited spatial muscle activation due to electrode placement. The effects of FES were further simulated by constraining all of the multiple elements representing a stimulated muscle in the model to have the same level of activation. In the case of non-selective nerve cuff electrodes, the constraint included all elements in all muscles stimulated by the same nerve cuff electrode.

The model was used to run inverse dynamic simulations including different sets of stimulating electrodes. The inputs to these simulations were movements expressed as patterns of joint angles, and the outputs were the required muscle activations. Simulations of different movements succeeded or failed, either for part of a movement or for the entire duration. Failure was due either to insufficient muscle force to overcome the mass of the arm, or the inability to maintain glenohumeral or scapulothoracic stability. For each movement, the 
success rate of an electrode set was defined as the ratio of the number of successful time steps to the total number of time steps for that movement.

The movements that were simulated were humeral elevation in different planes (frontal, sagittal, scapular), elbow flexion-extension, forearm pronation-supination, reaching movements to different heights, and activities of daily living (ADL) such as eating and combing the hair. These movements were recorded from one able-bodied subject using an Optotrak system (Northern Digital Inc.). This system includes three cameras that trace the 3D positions of light emitting diodes (LED) located within the workspace. In order to trace the position of the thorax, humerus and forearm of the subject, sets of LED were fixed over these segments. The humeral position can be used to estimate the locations of the scapula and clavicle, which are difficult to accurately measure during movement, using the well-defined relation between scapular and humeral motion (de Groot and Brand 2001). Using the positions of scapular and humeral bony landmarks recorded in a series of static trials throughout the workspace, a three-dimensional regression model relating humeral position to scapular and clavicular positions was built. This regression model was then used for the movement trials to estimate the dynamic orientations of the scapula and the clavicle from humeral position measurements. Data were recorded at $30 \mathrm{~Hz}$.

The various peripheral nerves (for nerve cuff electrodes) and muscles (for musclebased electrodes) that are candidates for stimulation are presented in table 3-1. In order to reduce the number of candidate sets, the first step was to determine which of these muscles and nerves are absolutely essential for the simulated movements. This was done by removing one muscle or nerve at a time from a best-case scenario set that includes all the candidates individually stimulated. If removal of a particular muscle or nerve caused the success rate to 
decrease significantly from the best-case scenario set (i.e., to near zero for one or more of the movements), that muscle or nerve was considered to be essential, and was included in all subsequent stimulation sets.

\begin{tabular}{|c|c|c|c|}
\hline \multicolumn{4}{|c|}{ Nerve Cuff Electrodes } \\
\hline Nerve & Selective & Muscles Innervated & $\begin{array}{l}\text { Branch that can be } \\
\text { avoided }\end{array}$ \\
\hline Radial & yes & $\begin{array}{l}\text { Triceps, anconeus, brachioradialis, } \\
\text { supinator, wrist and finger muscles }\end{array}$ & Long head of triceps \\
\hline Musculocutaneous & maybe & $\begin{array}{ll}\text { Biceps, brachialis } & \text { and } \\
\text { coracobrachialis }\end{array}$ & Coracobrachialis \\
\hline Axillary & maybe & Deltoid and teres minor & Teres Minor \\
\hline Suprascapular & maybe & Infraspinatus and supraspinatus & \\
\hline Upper subscapular & no & Subscapularis & \\
\hline Long Thoracic & no & Serratus anterior & $\begin{array}{l}\text { Upper heads of the } \\
\text { serratus anterior }\end{array}$ \\
\hline Thoracodorsal & no & Latissimus dorsi & \\
\hline \multicolumn{4}{|l|}{ Muscle Electrodes } \\
\hline & & Rhomboids & \\
\hline & & Upper and lower pectoralis major & \\
\hline & & Pronator quadratus & \\
\hline & & Supinator & \\
\hline
\end{tabular}

Table 3-1: Nerves and muscles candidate for stimulation.

Once the essential muscles and nerves were identified, a second set of simulations was run in order to determine which non-essential muscles should be stimulated to maximize performance within the allocated number of stimulation channels. These simulations also examined the best options for the nerve cuff electrodes, i.e. whether they should be placed proximally or distally to a muscle branch and which ones should be selective. These options are also shown in table 3-1. Because of the limited number of stimulation channels in our neuroprosthetic system (14 for shoulder and arm muscles), there can only be two selective cuff electrodes (each selective cuff requires four stimulation channels). One of the selective cuff electrodes was reserved for the radial nerve because it innervates both extensors (triceps, 
anconeus) and flexors (brachioradialis) of the elbow, as well as wrist and finger muscles, providing opportunities for restoring multiple functions from a single cuff while reducing the likelihood of unintended activation of multiple muscles. The remaining selective cuff was evaluated on all other targeted nerves that innervated more than one muscle (i.e., musculocutaneous, axillary, suprascapular). Based on these different options, all possible electrode sets were created, and inverse dynamic simulations were run by the model. The set with the highest mean success rate across movements was chosen as the targeted set of the neuroprosthetic system.

\section{Results}

Figure 3-1 shows an example of a model simulation and subsequent calculation of the success rate, for a 15 -second humeral elevation movement in the coronal plane. The humeral elevation angle is shown on the top left. Note that this is only one of the eleven different joint angles measured from the able-bodied subject and used an inputs to the model. The muscle set included for this simulation is shown on the right. This set includes selective cuffs on the axillary and radial nerves, non-selective cuffs on the musculocutaneous, suprascapular and long thoracic nerves, and muscle electrodes on the pectoralis major, pronator quadratus and supinator. The activation pattern calculated by the model for one of the stimulation channels (the cuff on suprascapular nerve) is pictured on the bottom left. As indicated by the grey bars, the simulation failed for elevation angles above 80 degrees. Out of 450 total timesteps, 247 were successful, which gives a success rate of 0.55 . 


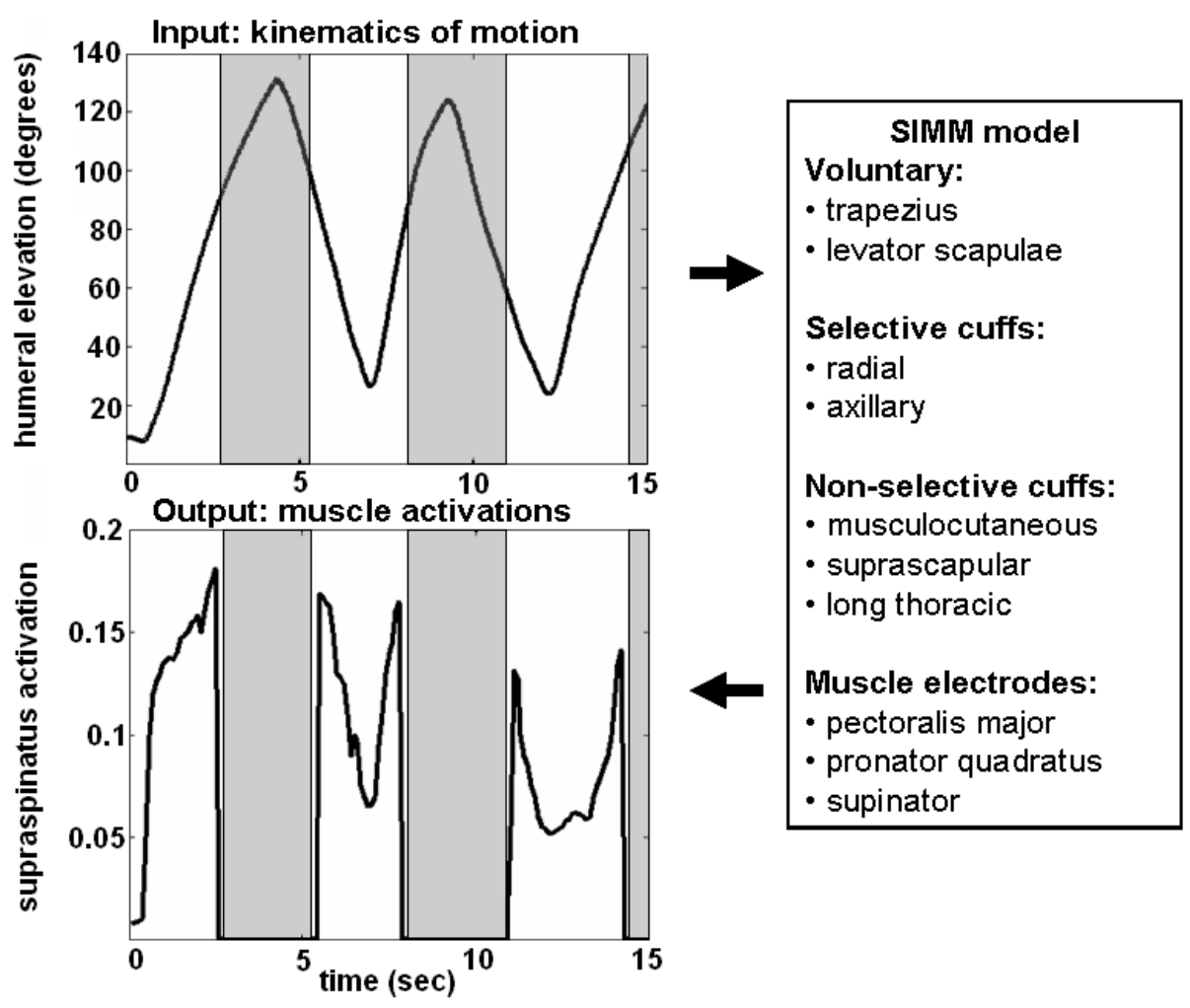

Figure 3-1: Inputs and outputs of the musculoskeletal model, and calculation of simulation success rate. As an example, a humeral elevation movement in the coronal plane is shown. On the top left is the humeral elevation angle, on the right is the electrode set, and on the bottom left is the calculated activation pattern for the suprascapular nerve cuff. The grey bars highlight the portions of the simulation that failed.

Table 3-2 summarizes the results of the first set of simulations, aiming at identifying the "essential" nerves and muscles for stimulation. The top row shows the success rates for all movements for the "best case" scenario, i.e., the set that included all the candidate muscles individually stimulated and all the candidate nerves stimulated selectively. The long thoracic nerve is a special case: although it innervates only one muscle, the serratus anterior, stimulation of the entire muscle at the same level causes almost complete failure of all movements, as shown in the second row of Table 3-2 (mean success rate: 0.02 ). If only the lower half of the muscle is stimulated (simulating a more distal cuff location along the long 
thoracic nerve), the mean success rate is increased substantially (to 0.83 ). Therefore, the bestcase scenario set (top row) includes stimulation of only the lower half of serratus anterior. Since the muscle strengths are constrained to $50 \%$ of able-bodied strength, even in the bestcase scenario the FES system can only restore $83 \%$ and not $100 \%$ of the able-bodied movements.

\begin{tabular}{|c|c|c|c|c|c|c|c|c|c|c|c|}
\hline & 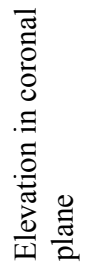 & 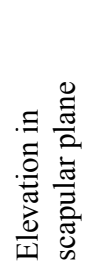 & 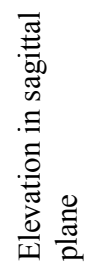 & 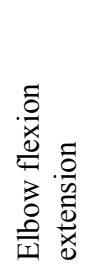 & 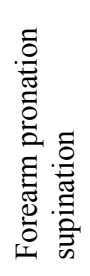 & 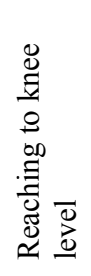 & 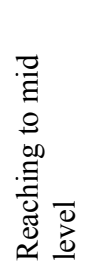 & 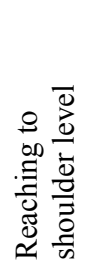 & 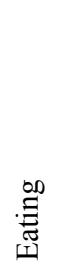 & 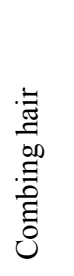 & 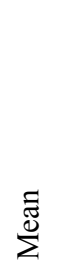 \\
\hline $\begin{array}{l}\text { Best-case } \\
\text { scenario }\end{array}$ & 0.81 & 0.84 & 0.79 & 0.99 & 0.92 & 0.93 & 0.90 & 0.60 & 0.94 & 0.53 & 0.83 \\
\hline $\begin{array}{l}\text { all serratus } \\
\text { anterior }\end{array}$ & 0.03 & 0.03 & 0.00 & 0.06 & 0.05 & 0.02 & 0.01 & 0.01 & 0.00 & 0.01 & 0.02 \\
\hline \multicolumn{12}{|l|}{ Exclude: } \\
\hline serratus anterior & 0.00 & 0.00 & 0.00 & 0.00 & 0.00 & 0.00 & 0.00 & 0.00 & 0.00 & 0.00 & 0.00 \\
\hline radial & 0.28 & 0.17 & 0.16 & 0.00 & 0.05 & 0.03 & 0.02 & 0.02 & 0.00 & 0.00 & $\mathbf{0 . 0 7}$ \\
\hline $\begin{array}{l}\text { Musculo- } \\
\text { cutaneous }\end{array}$ & 0.31 & 0.20 & 0.23 & 0.00 & 0.01 & 0.05 & 0.04 & 0.01 & 0.00 & 0.00 & 0.08 \\
\hline axillary & 0.10 & 0.12 & 0.11 & 0.97 & 0.92 & 0.45 & 0.24 & 0.13 & 0.62 & 0.10 & 0.38 \\
\hline suprascapular & 0.09 & 0.24 & 0.23 & 0.95 & 0.88 & 0.49 & 0.20 & 0.13 & 0.52 & 0.14 & 0.39 \\
\hline upper subscapular & 0.67 & 0.69 & 0.61 & 0.98 & 0.92 & 0.80 & 0.79 & 0.47 & 0.77 & 0.48 & 0.72 \\
\hline thoracodorsal & 0.80 & 0.84 & 0.79 & 0.99 & 0.92 & 0.92 & 0.90 & 0.60 & 0.94 & 0.53 & 0.82 \\
\hline pectoralis major & 0.75 & 0.42 & 0.01 & 0.99 & 0.92 & 0.78 & 0.55 & 0.43 & 0.68 & 0.22 & 0.58 \\
\hline rhomboid & 0.78 & 0.81 & 0.70 & 0.99 & 0.92 & 0.93 & 0.89 & 0.57 & 0.94 & 0.50 & 0.80 \\
\hline supinator & 0.81 & 0.84 & 0.79 & 0.85 & 0.02 & 0.73 & 0.35 & 0.23 & 0.21 & 0.29 & 0.51 \\
\hline $\begin{array}{l}\text { pronator } \\
\text { quadratus }\end{array}$ & 0.81 & 0.83 & 0.77 & 0.60 & 0.00 & 0.62 & 0.60 & 0.44 & 0.20 & 0.27 & 0.51 \\
\hline
\end{tabular}

Table 3-2: The success rate of different electrode sets for every simulated movement. The first row represents results from the best-case scenario, which consists of all candidate muscles or nerves individually stimulated, but including only the lower half of the serratus anterior. The second row shows the success rates of the same set but including the entire serratus anterior. The remaining rows correspond to the best-case scenario set excluding one muscle or nerve at a time. The most dramatic decreases in the success rate are highlighted by gray shading and bold text.

The rest of table 3-2 illustrates the effects on the success rate when every muscle or nerve was removed one at a time from the best-case scenario set. It is clear that the success rate of certain movements was near zero if certain individual electrodes were removed. For 
example, omitting the electrodes on the upper and lower pectoralis major from the stimulation set produced a success rate of just 0.01 for movements that required arm elevation in the sagittal plane. Other electrodes were essential for all movements, like the cuff on the radial nerve, without which the mean success rate decreased to 0.07 . Based on these results, the essential electrodes are the cuffs on the long thoracic, radial, musculocutaneous, axillary and suprascapular nerves, and muscle electrodes on the pectoralis major, supinator and pronator quadratus. Removing the electrodes from either the rhomboids or the thoracodorsal nerve did not have a significant effect on the success rate (mean of 0.80 and 0.82 , respectively, vs. 0.83 for the best-case scenario), while removing the cuff on the upper subscapular nerve (which innervates the subscapularis) caused a $12 \%$ reduction of the mean success rate $(0.72$ from 0.83$)$. These three electrodes were considered to be nonessential.

Once the essential set was determined, all possible stimulation sets (a total of 48) were created based on the maximum number of channels in the proposed system (14), the inclusion or not of the non-essential electrodes, and the nerve cuff electrode options mentioned earlier (i.e. selective, non-selective, placed proximally or distally to a muscle branch). Those sets were used in simulations with all the recorded movements as inputs, and the success rate was calculated for each one. The electrode set with the highest mean success rate is shown in table 3-3. This set includes two selective nerve cuff electrodes, four nonselective nerve cuff electrodes, and five muscle based electrodes. In addition, the nerve cuff channels include specific locations along several of the peripheral nerves that are distal to the nerve branches for undesirable muscles. The optimal radial nerve cuff location was distal to the nerve branch to the long head of triceps, the optimal axillary nerve cuff location 
was distal to the nerve branch to the teres minor, and the optimal musculocutaneous nerve cuff location was distal to the branch to the coracobrachialis. Table 3-4 shows the success rate of this optimal electrode set for all the simulated movements. The mean success rate across all movements was 0.40 .

\begin{tabular}{|l|l|l|c|l|}
\hline Nerve Cuff Electrodes & Muscles Stimulated & $\begin{array}{l}\text { Number of } \\
\text { channels }\end{array}$ & $\begin{array}{l}\text { Muscles that must } \\
\text { be avoided }\end{array}$ \\
\hline Nerve & Selective & 3 & Long head of triceps \\
\hline Radial & yes & Medial and lateral heads of triceps & 2 & Coracobrachialis \\
\hline Musculocutaneous & yes & Biceps and brachialis & 1 & Teres Minor \\
\hline Axillary & no & Deltoid & 1 & \\
\hline Suprascapular & no & Infraspinatus and supraspinatus & 1 & \\
\hline Upper subscapular & no & Subscapularis & 1 & $\begin{array}{l}\text { Upper part of } \\
\text { serratus anterior }\end{array}$ \\
\hline Long Thoracic & no & Lower part of serratus anterior & \multicolumn{2}{|c|}{} \\
\hline & \multicolumn{2}{|c|}{ Muscle Electrodes } & 1 & \\
\hline & Rhomboids & 2 & \\
\cline { 2 - 4 } & Upper and lower pectoralis major & 1 & \\
\cline { 2 - 4 } & Pronator quadratus & 1 & \\
\cline { 2 - 4 } & Supinator & & \\
\cline { 3 - 4 } &
\end{tabular}

Table 3-3: The optimal electrode set. This includes muscle electrodes, selective, and non-selective nerve cuff electrodes. When important, the placement of the electrode on the nerve is specified.

\begin{tabular}{|l|l|}
\hline Movements & Success rate \\
\hline Humeral elevation in coronal plane & 0.27 \\
\hline Humeral elevation in scapular plane & 0.15 \\
\hline Humeral elevation in sagittal plane & 0.13 \\
\hline Elbow flexion-extension & 0.82 \\
\hline Forearm pronation-supination & 0.76 \\
\hline Reaching to knee level & 0.65 \\
\hline Reaching to mid level & 0.47 \\
\hline Reaching to shoulder level & 0.15 \\
\hline Eating & 0.49 \\
\hline Combing hair & 0.13 \\
\hline Mean & 0.40 \\
\hline
\end{tabular}

Table 3-4: The success rate of the optimal electrode set for every simulated movement. 
As noted above, we had already reserved one of two possible selective cuff electrodes for the radial nerve. The simulation results were used to choose the second selective cuff electrodes from among the axillary, musculocutaneous, and suprascapular nerves. The remaining two nerves would then receive nonselective cuff electrodes, along with the long thoracic and upper subscapular nerves (which would not benefit from a selective cuff since each serves only a single muscle, serratus anterior and subscapularis, respectively). Figure 32 shows the success rates for the three different selections of the second selective cuff. These three sets have the highest mean success rates among all the tested muscle sets that included the second selective cuff on the indicated nerve. The black bars indicate the success rates when the axillary nerve cuff was selective, the gray bars when the musculocutaneous nerve cuff was selective, and the white bars when the suprascapular nerve cuff was selective. Although use of a selective axillary nerve cuff provided the highest success rate for humeral elevation in all planes, the use of a selective musculocutaneous cuff electrode provided much greater success rates for almost all other movements. The overall average success rate for the selective musculocutaneous nerve cuff $(0.40)$ was nearly twice that of the selective axillary nerve (0.21) and more than three times that of the selective suprascapular nerve cuff $(0.12)$. 


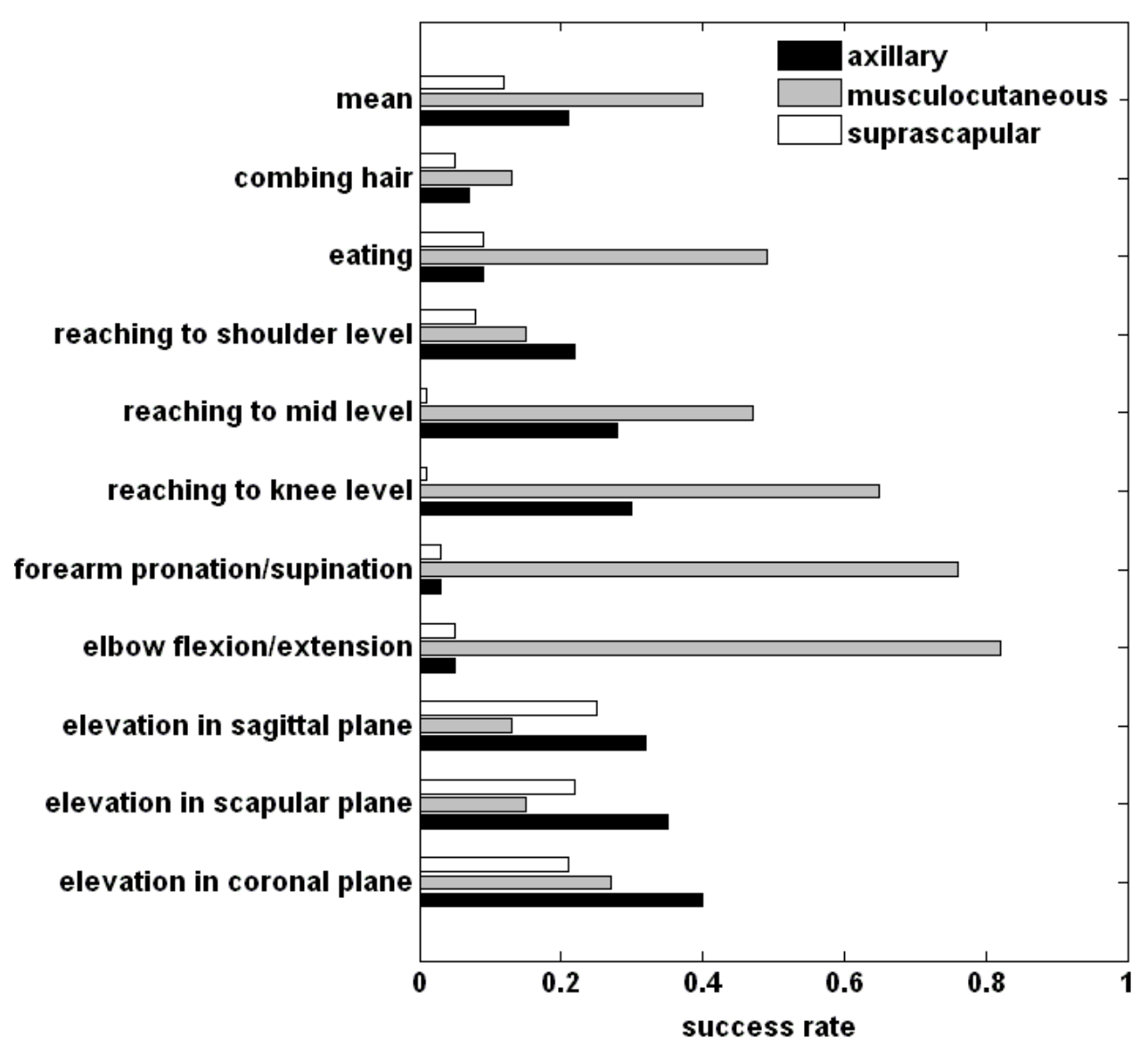

Figure 3-2: The effect of different selective and non-selective nerve cuff electrodes. The radial nerve was simulated with a selective cuff in all cases. The horizontal bars indicate the success rate for different selections of a second selective nerve cuff electrode: axillary nerve (black bar), musculocutaneous (grey bar), or suprascapular (white bar). The mean success rate is shown on the top, and the success rates for all movements individually are shown underneath. 


\section{Discussion}

This study used a musculoskeletal model to evaluate different stimulated muscle sets for restoration of upper extremity motor function in high tetraplegia. The consequences of a high level injury on the muscle function, as well as the application of FES using different electrode types were simulated in the model as realistically as possible using constraints in the muscle forces and activation patterns. Moreover, practical constraints such as the number of stimulating channels available in the proposed neuroprosthesis, and anatomical constraints regarding the feasible electrode positions were taken into account to propose a muscle set that can be a reasonable target for the FES system.

The muscle set identified as the optimal includes "prime movers" of the shoulder (deltoid, pectoralis major), muscles that stabilize the shoulder (infraspinatus, supraspinatus, subscapularis, serratus anterior, rhomboids), muscles for elbow flexion-extension (biceps, brachialis, medial and later triceps), and muscles for forearm pronation-supination (pronator quadratus, supinator). This muscle set has relatively high success rates for simple movements of the elbow and forearm (0.76-0.82), and reaching to a low level, like a tabletop $(0.65)$. The success rate was the lowest $(0.13-0.15)$ for movements that require significant humeral elevation, like combing hair, or reaching for something at shoulder level. This probably results from the mechanical actions of the non-selective cuff on the axillary nerve that activates all heads of the deltoid at the same level. The deltoid is a wide muscle, and even though its primary action is humeral elevation, the lowest fibers of the anterior and posterior part actually pull the humerus downwards. Another effect of the non-selective cuff for the deltoid is that movement in the sagittal plane, which requires more anterior than posterior deltoid, is degraded. This can be seen in table 3-4 by comparing the success rate for elevation 
in the sagittal plane (0.13) and coronal plane (0.27). A selective cuff on the axillary nerve that would allow the three heads of the deltoid to act separately would increase the success rate for humeral abduction, as was indicated in Figure 3-2.

The number of selective cuff electrodes in this system was constrained to two, however, because of the limited number of available channels. Even though a selective cuff on the axillary nerve would increase the range of motion to higher elevation levels, a selective cuff on the musculocutaneous nerve was shown to be critical for elbow control, which is important for many activities of daily living such as eating. This is shown in Figure 3-2, where a non-selective cuff on the musculocutaneous nerve results in success rates for elbow flexion-extension and eating of less than 0.1 . The biceps and brachialis are both elbow flexors, but the biceps also acts on the scapula (for humeral flexion) and on the radius (for forearm supination). The simulations showed that the difference in muscle action between biceps and brachialis is so significant that effective control of the elbow is prevented by their simultaneous activation.

Cuff electrode placement was found to be another important factor for the success of the simulated movements. The teres minor causes external rotation of the humerus, which is not always desirable during abduction movements performed by the deltoid. As a result, the optimal axillary nerve cuff location was found to be distal to the teres minor. In a similar way, the long head of the triceps extends the shoulder as well as the elbow, preventing the coordination of the shoulder and elbow joints. Finally, the coracobrachialis produces shoulder flexion that was found to be disadvantageous when coupled to the elbow flexion actions of the other muscles innervated by the musculocutaneous nerve (biceps, brachialis). 
The impact of electrode placement was particularly dramatic for the long thoracic nerve (serratus anterior muscle). Table 3-2 shows that stimulation of the entire serratus anterior, a very important muscle for scapular stability, causes simulation failure in all cases. This is due to the wide origin and attachment of the muscle (from the upper eight or nine ribs to the entire medial border of the scapula), which results in different fibers having different actions (scapular protraction, upward rotation, or depression). This problem is avoided if the cuff is placed more distally on the long thoracic nerve, so that it only stimulates the lower half of this muscle. This activation preserves the scapular stability function of the serratus anterior without the other actions of its more superior fibers.

Muscle selection in this study was based on a model of a generic high tetraplegia subject. Even though the model parameters, some of which were measured from cadaver studies (skeletal dimensions and masses, muscle attachment sites) and some of which were assumed (e.g., 50\% maximum forces in paralyzed but stimulated muscles), do not correspond perfectly with those of a particular FES participant, meaningful conclusions can still be reached about the muscles that would be most effective in restoring movements to paralyzed individuals. With information from a particular individual, this technique could result in a neuroprosthetic system that is further customized to the person's specific needs. For example, MR imaging techniques could be used to estimate the maximum forces expected from paralyzed muscles (Fukunaga et al. 1992). In addition, the simulations could focus on the movements needed to achieve the particular functional goals of a specific person. In cases of extensive muscle denervation, a mobile arm support could be simulated and added to the arm model, and then used in simulation to investigate the effect of combined FES and orthotic support against gravity. 
The success rate of even the optimal electrode set chosen based on the model simulations was only $40 \%$ across all movement types. Movements that require humeral elevation at the shoulder level or above, reaching straight to the front and across the body, and extreme pronation and supination will not be possible with existing FES technology because of the number of stimulation channels required. However, for a person with complete paralysis of the upper extremity, even the relatively limited movements made possible by our proposed FES system would represent a significant improvement in the quality of life. 


\title{
Chapter 4: Combined feedforward and feedback control of a redundant, non-linear, dynamic musculo-skeletal system.
}

\begin{abstract}
A functional electrical stimulation (FES) system is under development for the restoration of function to individuals with high cervical level spinal cord injury. With most of the upper extremity paralyzed, the FES system needs to appropriately stimulate a large number of shoulder and elbow muscles, in order to restore functional movements. This study proposes the use of a combined feedforward and feedback controller for this system. The feedforward controller generates the muscle activations required for a desired movement, and the feedback controller corrects for errors caused by muscle fatigue and external disturbances. The feedforward controller is an artificial neural network (ANN) trained on the dynamics of the human arm. The feedback loop includes a PID controller in series with a second ANN trained on the steady-state nonlinear properties and biomechanical interactions of muscles and joints. The controller is designed and tested using a simplified musculoskeletal model of the upper extremity that includes two joints, four mono-articular and two bi-articular muscles. Its performance is evaluated during goal-oriented movements of varying amplitudes and durations, with and without fatigue and external forces. The controller has a tracking error of less than 4 degrees in ideal conditions, and less than 10 degrees even in the case of considerable fatigue and external disturbances.
\end{abstract}

\section{Introduction}

Functional electrical stimulation (FES) systems restore function after spinal cord injury by stimulating paralyzed muscles. The controller is the part of the FES system that 
calculates the stimulation patterns that need to be applied to the appropriate muscles in order to perform the desired function. This is a complicated task, because the musculoskeletal system is highly non-linear, time-varying and redundant (Chizeck and Kobetic 1988). We are interested in control of an FES system for restoration of shoulder and arm function to individuals with high cervical level spinal cord injury. The additional challenge for the control of upper extremity tasks is that they are goal directed, unlike the cyclic movements of the lower extremity (Crago et al. 1996). This means that the amplitude, speed and direction of the motions change continuously, so the controller needs to continuously adjust the stimulation patterns for the motions to be performed.

There are different control strategies used in FES systems. Feedforward control is commonly used in clinical practice (Jonic and Popovic 1997; Kilgore et al. 1989). The output of this type of controller depends only on the user command, and not on the system performance. The advantage of this design is that it does not need sensors to measure the system output, but the disadvantage is that it is unable to make corrections if the actual movement deviates from the desired movement. Feedback control uses sensors to monitor the system output, so it can correct for errors in the system trajectory (Crago et al. 1996). Feedback is necessary in order to maintain good tracking performance in the presence of fatigue and possible external disturbances. However, inherent delays in the response of the system can cause problems to the feedback controller (Abbas and Chizeck 1995), especially in the case of fast movements (Stroeve 1996). Therefore, a combination of feedforward and feedback control has the best results, and it is the preferred control method in many FES system designs (Abbas and Chizeck 1995; Chang et al. 1997; Pedrocchi et al. 2006), including the one presented in this paper. 
The feedforward controller is typically an inverse-dynamic model of the controlled system (Norgaard et al. 2000). Since an actual inverse-dynamic model is usually not available, data collected from the system itself are used to train an artificial neural network (ANN) to behave like the inverse-dynamic model. An ANN can approximate any dynamic system based solely on the knowledge of inputs and outputs (Norgaard et al. 2000). In order to train the network to represent the inverse dynamics of the system, inputs and outputs are recorded from the system and used as the outputs and inputs, respectively, of the ANN. This has been used in many FES controllers of single-joint, single-muscle systems (Chang et al. 1997; Pedrocchi et al. 2006; Yoshida et al. 2002; Abbas and Triolo 1997; Adamczyk and Crago 2000). However, in the case of a system with multiple muscles crossing one joint, the inverse of the system is not unique, and the ANN will train on the average of possible solutions, which is not always a correct solution (Karniel and Inbar 2000).

For this reason, in our approach we have used the actual inverse-dynamic model of the system to train the ANN in the feedforward component of our controller. Optimization was used to ensure uniqueness of the solution. In Ferrarin et al. (2001), the inverse-dynamic model itself was used as a feedforward controller, but a relatively simple model had to be designed so that it was fast enough to run in real-time. An ANN, however, is a system of simple processing elements, connected into a network by a set of weights (Norgaard et al. 2000), so it is computationally light. Off-line training of the ANN according to the inversedynamic model allows us to include all the complexities of the musculoskeletal system in a real-time controller.

For the feedback part of the controller, PID controllers are widely used in FES (Abbas and Chizeck 1995; Chang et al. 1997; Veltink et al. 1992). However, their 
performance has been limited, as discussed in Pedrocchi et al. (2006), because PID controllers, being linear, are unsuitable for the highly non-linear nature of musculoskeletal systems (Astrom and Hagglund 2001). Moreover, the PID controllers mentioned above were all tested in single-joint, single-muscle systems; the complexity of musculoskeletal systems increases when they include more degrees of freedom and more muscles, some of them biarticular, that cause mechanical coupling between joints (Adamczyk and Crago 2000). A multivariable feedback controller that uses PI control is described by Lan et al. (1991), but its performance is only evaluated in isometric conditions. The authors suggest that nonlinear control methods may be necessary for control of movements of a multi-joint system.

In order to address these issues, we have created a neuro-PID controller for the feedback loop, which combines the attractive features of PID control (robustness and ease of implementation) with the nonlinear nature of ANN. The output of the PID controller becomes the input to an ANN trained as a steady-state inverse model. Its goal is to model the nonlinear relationships among muscles and joints, allowing the PID controller to deal solely with the dynamic response.

The feedforward-feedback FES controller presented in this paper is designed and tested in an arm model that includes two joints, and six muscles, two of them bi-articular, in order to ensure it can handle the complicated interactions between multiple muscles and joints. It is evaluated in a large set of goal-directed movements that cover the range of both joints. Muscle fatigue and external disturbances are also simulated, to evaluate the performance of the controller in realistic conditions. 


\section{Methods}

The model

The model used to design and test the controller is a two-dimensional model of the upper extremity, in the horizontal plane (no gravity). It includes six muscles (anterior and posterior deltoid, long head of the biceps, brachialis, long and lateral head of the triceps) and two degrees of freedom (shoulder flexion-extension and elbow flexion-extension). The range of the shoulder angle is from -20 to 110 degrees, and the range of the elbow angle is from 0 to 170 degrees. Four of the muscles are mono-articular, and two (long head of biceps and long head of triceps) are bi-articular. A schematic of the model is shown in figure 4-1.

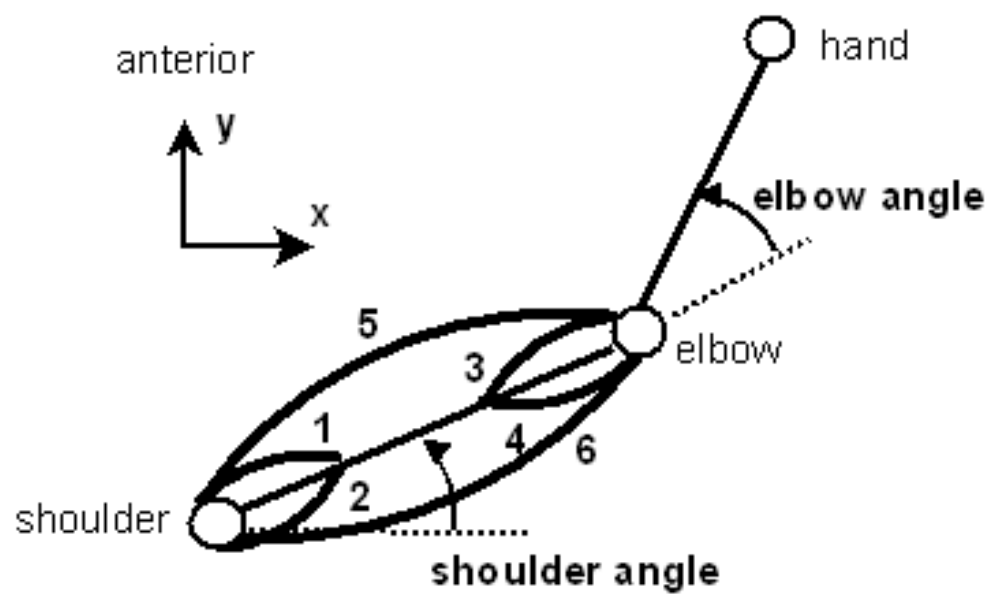

Figure 4-1: The model. It has two degrees of freedom (shoulder flexion-extension and elbow flexion-extension), and six muscles numbered in the figure: 1 . anterior deltoid, 2. posterior deltoid, 3. brachialis, 4. lateral triceps, 5. long head of biceps, and 6. long head of triceps. 1 and 2 cross the shoulder joint, 3 and 4 cross the elbow joint, and 5 and 6 cross both joints. 
The muscle and joint parameters for the model were obtained from cadaver studies by Klein-Breteler et al. (1999). These parameters include the position of joint centers, inertial parameters for body segments, and the optimal fiber length, origin and insertion, tendon slack length, and physiological cross-sectional area of every muscle. The muscle model is a Hilltype model that includes contraction dynamics, force-length dependence and force-velocity dependence. It was developed by McLean et al. (2003).

The model can run both forward and inverse dynamic simulations. In the forward dynamic mode, the inputs are the activations of the six muscles, and the outputs are the angles and angular velocities of the two joints. In the inverse dynamic mode, the inputs are the desired angles and angular velocities of the two joints, and the outputs are the required muscle activations. Both modes are used in the controller design and testing, as described in the next section. In the case of inverse dynamic simulations, an optimization routine is needed in order to distribute the muscle forces based on the required joint torques, since there are more muscles than degrees of freedom. The objective function currently used was proposed by Praagman et al. (2006) to minimize energy consumption:

$$
E_{m}=E_{f}+E_{a}=m\left\{c_{1} \frac{F_{m}}{P C S A}+c_{2}\left(\frac{F_{m}}{F_{\max }}\right)^{2}\right\}
$$

where $E_{f}$ and $E_{a}$ are the muscle energy consumption due to the detachment of cross bridges and re-uptake of calcium respectively, $m$ is the muscle mass, $F_{m}$ is the muscle force, PCSA is the physiological cross-sectional area, $F_{\max }$ is the maximum muscle force, and $c_{1}$ and $c_{2}$ are two constants chosen such that 50-50 contribution from the linear and nonlinear terms at 50\% activation is reached (Praagman et al. 2006). 


\section{The controller}

The controller consists of a feedforward and a feedback part (figure 4-2). The feedforward part is an ANN trained to behave like the inverse-dynamic model of the arm, so that it can generate the muscle activations required for a desired movement, based on knowledge of system dynamics. This ANN is a two-layer network with a sigmoidal transfer function in the hidden layer, and a linear transfer function in the output layer. The inputs are the shoulder and elbow angles, and four past values of the angles, used to estimate angular velocity. The outputs are activation levels for the six muscles.

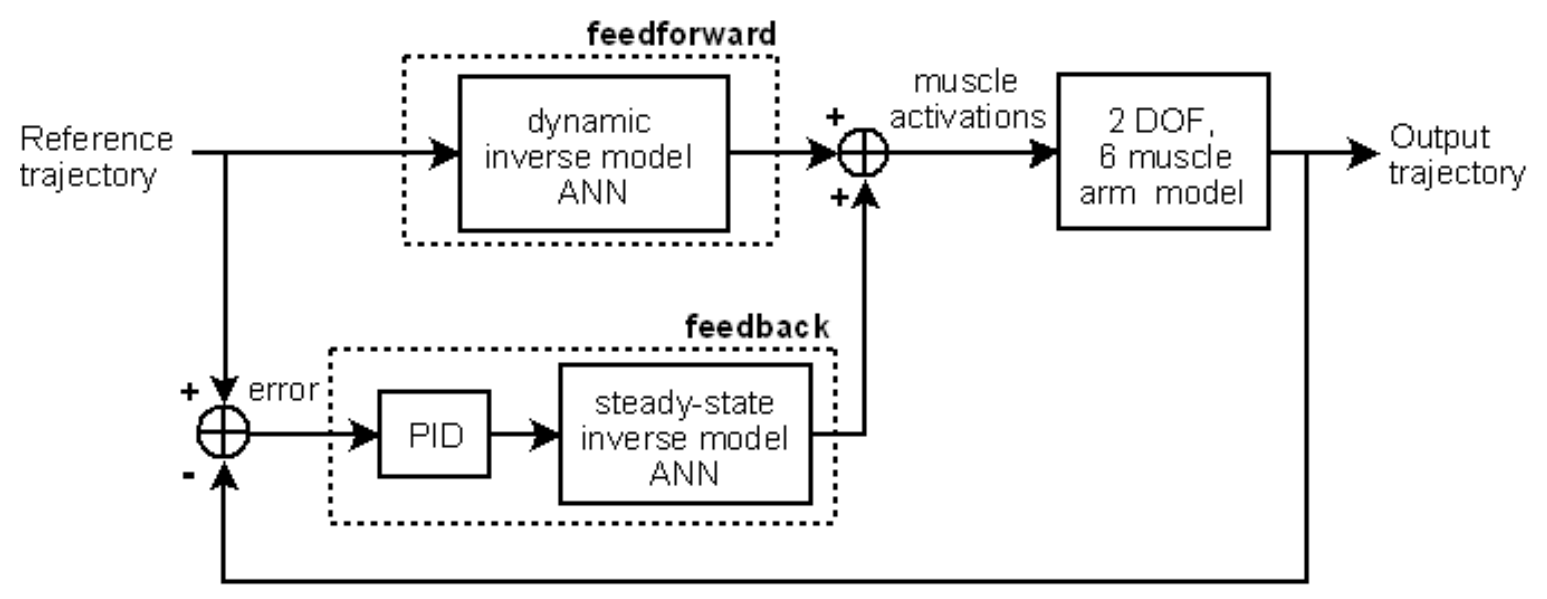

Figure 4-2: The combined feedforward and feedback $(\mathrm{FF}+\mathrm{FB})$ controller scheme.

Training of this ANN was done using data from inverse dynamic simulations using the model. The movements used for training were trajectories covering the range of both shoulder and elbow angles, with bell-shaped velocity profiles. This is the type of trajectory seen in goal-directed movements performed by able-bodied subjects (Happee and van der Helm 1995), and it covers a wide range of amplitudes and frequencies, which is a desired 
characteristic for ANN learning (Stroeve 1996). In order to improve learning, white noise with amplitude 5\% of the maximum angle was added to the input data, as described in Pedrocchi et al. (2006). The ability of the network to approximate the inverse-dynamic model was evaluated using the root mean squared error between the model and ANN predicted muscle activations:

$R M S E=\sqrt{\frac{\sum_{i=1}^{N}\left(y_{i}^{A N N}-y^{\bmod e l}{ }_{i}\right)^{2}}{N}}$

Networks with different numbers of neurons in the hidden layer were trained, and the network with 15 neurons had the smallest testing RMS error: 0.07 (no units, this is normalized muscle activation ranging from 0 to 1 ). Consequently, this ANN was chosen as the feedforward part of the controller.

The feedback part of the controller was designed as a traditional PID controller in series with a static ANN. The PID controller is described by:

$u_{i}(t)=K_{P i} e_{i}(t)+K_{D i} \frac{d e_{i}(t)}{d t}+K_{I i} \int_{0}^{t} e_{i}(t), i=1,2$

where $\mathrm{K}_{\mathrm{Pi}}, \mathrm{K}_{\mathrm{Di}}$ and $\mathrm{K}_{\mathrm{Ii}}$ are the proportional, derivative and integral gains for the shoulder $(\mathrm{i}=1)$ and the elbow (i=2). $\mathrm{u}_{1}$ and $\mathrm{u}_{2}$ are the inputs to the static ANN. The PID controller gains were tuned using the Ziegler-Nichols step response method (Astrom and Hagglund 2004) with additional manual fine-tuning. They are shown in table 4-1. 


\begin{tabular}{|c|c|c|c|}
\hline & $\mathrm{K}_{\mathrm{P}}$ & $\mathrm{K}_{\mathrm{I}}$ & $\mathrm{K}_{\mathrm{D}}$ \\
\hline shoulder & 2.5 & 3 & 1 \\
\hline elbow & 2.5 & 5 & 1 \\
\hline
\end{tabular}

Table 4-1: The PID gains used in the feedback part of the controller

The feedback ANN has the same architecture as the feedforward ANN (two layers, sigmoidal and linear transfer function in the hidden and output layer, respectively). However, this ANN is static, since it only uses the present angle values as inputs. In order to get training data, a 100 by 100 grid of shoulder and elbow angles was created, and inverse dynamic simulations were run with these angles as constant inputs. From each simulation, we recorded the set of muscle activations calculated by the model in the steady state. The angles and the corresponding muscle activations were used to train ANN with different numbers of neurons in the hidden layer, and the network with 10 neurons was chosen according to the RMS error. The error in this case was 0.09 .

The controller was tested using trajectories with bell-shaped velocity profiles, of different amplitudes and durations. Its performance was evaluated using the RMS error between the desired and actual trajectory. In order to investigate the effect of each part of the controller, the feedforward only (FF), feedback only (FB), and combination of feedforward and feedback $(\mathrm{FF}+\mathrm{FB})$ were tested separately.

In order to test the performance of the controller in imperfect conditions, a simple model of muscle fatigue was implemented in the model. The maximum muscle force is linearly reduced according to the equation:

$$
F_{j}(t)=F_{0 j}(t)(1-0.005 * t), j=1 . .6
$$


where $F_{0 j}$ is the maximum isometric force for muscle $\mathrm{j}$. Using a "fatigue rate" of 0.005 results in a $50 \%$ force reduction over 100 seconds, which has also been used by Abbas and Chizeck (1995) and Pedrocchi et al. (2006).

Finally, to test the ability of the controller to resist external disturbances, forces of different amplitudes were applied to the hand, in both the $\mathrm{x}$ (medial-lateral) and $\mathrm{y}$ (anterior posterior) directions. The amplitudes of the forces were drawn from a normal distribution with standard deviation $1 \mathrm{~N}$ and mean $1 \mathrm{~N}, 5 \mathrm{~N}, 10 \mathrm{~N}$ and $15 \mathrm{~N}$. The mean RMSE was calculated for each of the four cases.

\section{Results}

Figure 4-3 shows the performance of the FF, FB, and FF+FB controller for a 20second movement. Panel A shows the shoulder angle, and panel B shows the elbow angle. The RMS error for the three controllers was 8.3, 4.6, and 3.3 degrees respectively for the shoulder angle, and 8.4, 4.0, and 2.7 degrees respectively for the elbow angle.

The muscle activations corresponding to that movement are shown in figure 4-4. Panels A, B and C show the activations calculated by the FF, FB and FF+FB controller. Note that the feedforward muscle activations are much smoother than the feedback, resulting in a combination of smooth signals when the desired angles change slowly, and fast adjustments when the desired angles change rapidly. 

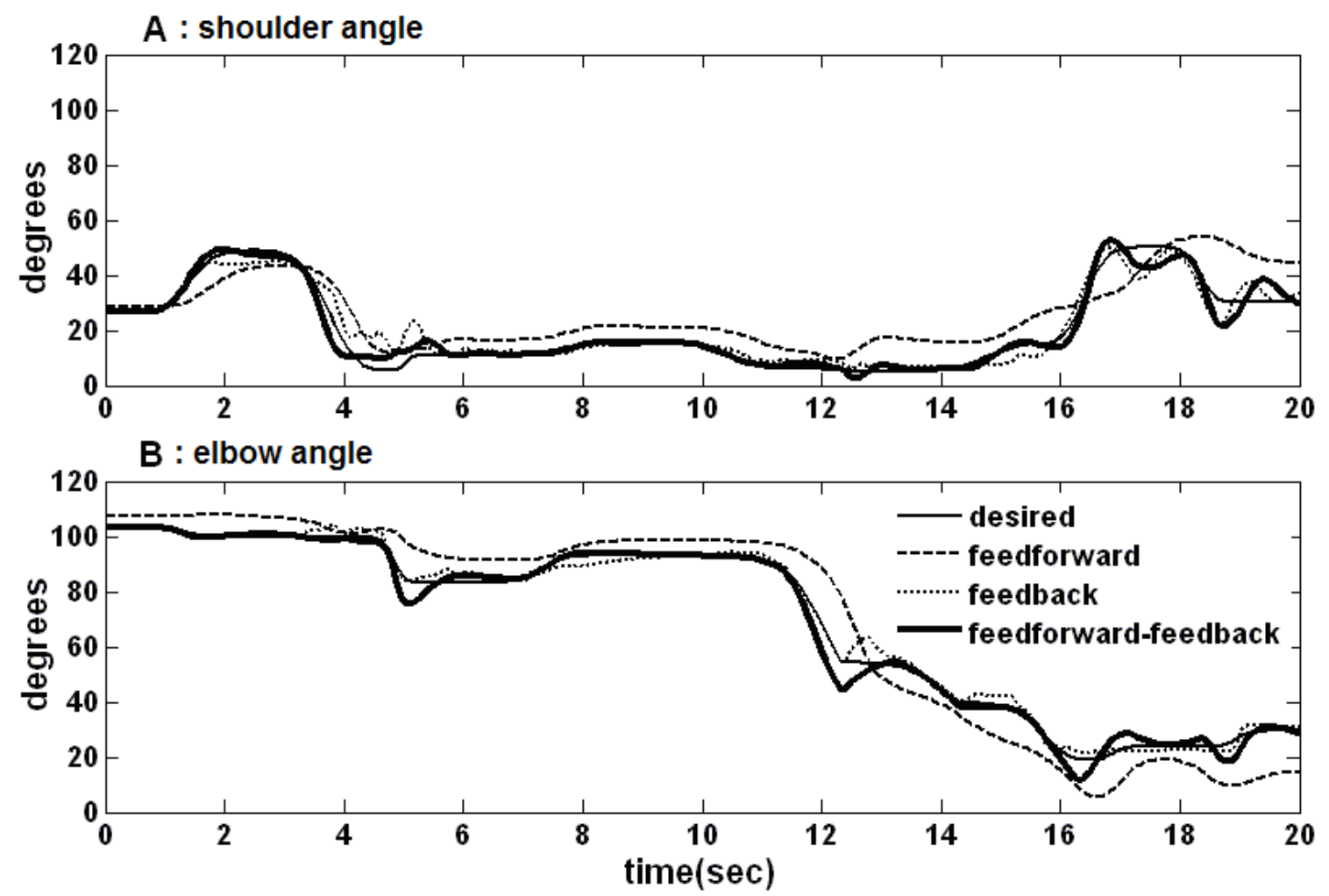

Figure 4-3: An example of the performance of the FF, FB and FF + FB controllers for a random movement. $\mathrm{A}$ is the shoulder and $\mathrm{B}$ is the elbow angle. 


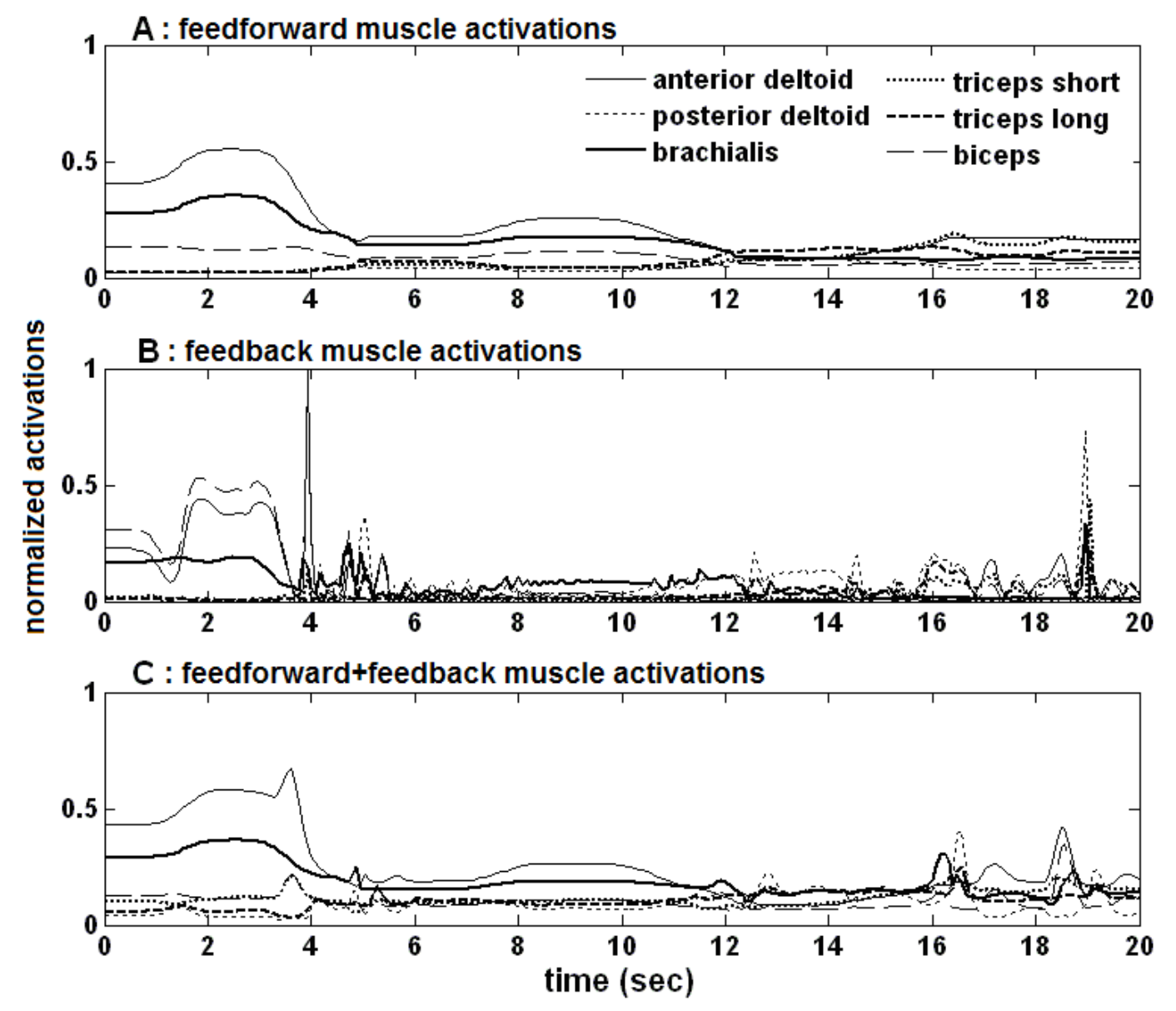

Figure 4-4: The muscle activations calculated for the movement presented in figure 4-3. $\mathrm{A}$ are the activations for the FF controller, $\mathrm{B}$ for the $\mathrm{FB}$, and $\mathrm{C}$ for the $\mathrm{FF}+\mathrm{FB}$ controller.

The mean tracking performance of the three controllers, calculated as the mean RMS error, is shown in Figure 4-5. The combination of feedforward and feedback has the best performance, with an RMS error of less than 4 degrees for both the shoulder and the elbow. The feedback controller has an RMS error of 5-6 degrees, and the feedforward controller has the worst tracking performance, with an RMS error of 15-20 degrees. 


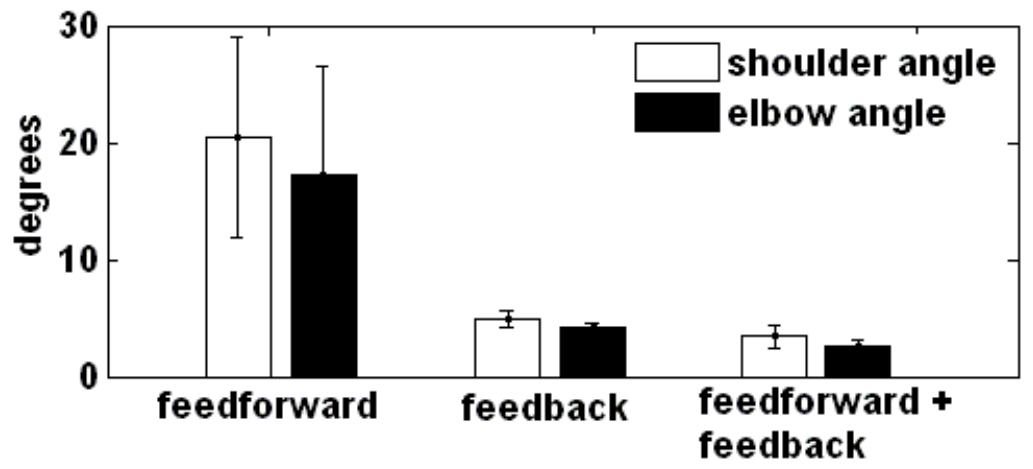

Figure 4-5: The mean RMS error across forty 60-second trials tested using the three controllers: FF, FB, and FF+FB.

Figure 4-6 shows an example of a repeated movement with decreasing muscle output to simulate fatigue, for all six muscles. The maximum muscle force is decreased by $0.5 \%$ per second, so by the end of the 190 -second movement, the maximum force is $5 \%$ of the initial maximum force. $\mathrm{A}$ is the shoulder and $\mathrm{B}$ is the elbow angle, and $\mathrm{C}$ and $\mathrm{D}$ are the errors (absolute difference between desired and output angles) for the shoulder and elbow respectively. Panel E shows the muscle activations calculated by the FF+FB controller. Note that the error for both the shoulder and elbow does not increase above 5 degrees until after 160 seconds of movement, which corresponds to a maximum muscle force of $20 \%$. Below that level, most activation levels have reached the maximum, as shown in panel $\mathrm{E}$, and the arm trajectory can no longer be controlled.

Figure 4-7 shows the mean RMS error across all trials with fatigue. The trials were split into 40 second intervals, and the RMS error was calculated for each one. For both the shoulder and the elbow, the RMS error increases as the fatigue level increases. During the last part of the trials, which corresponds to muscle force decreased to levels of $40 \%$ to $20 \%$, 
the RMS error is 10-15 degrees. However, the RMS error is less than 8 degrees for the first half of the trials, which corresponds to muscle force as low as $50 \%$.
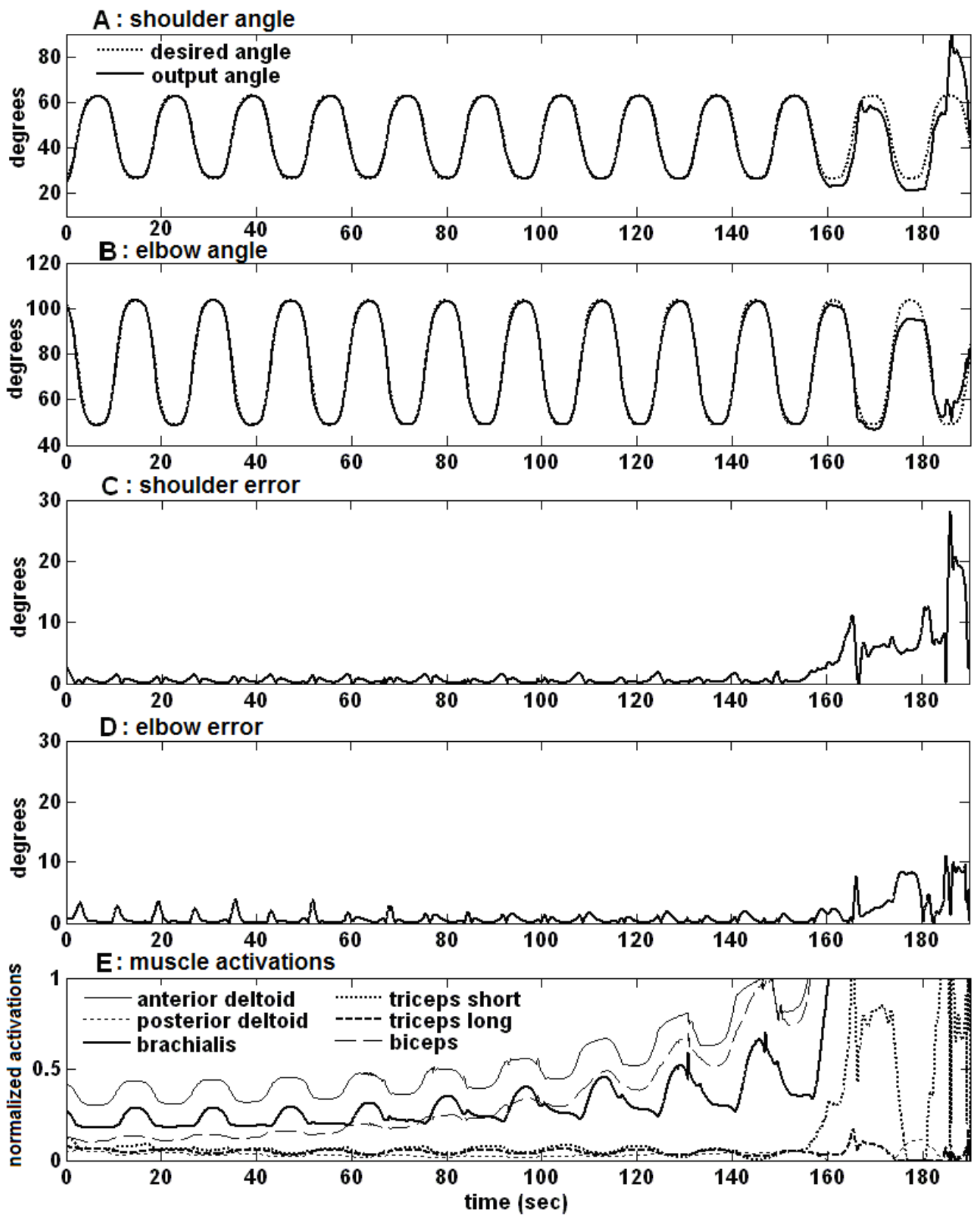

Figure 4-6: Example of movement with fatigue. The maximum muscle force is decreased by $0.5 \%$ per second, so by the end of the 190 -second movement, the maximum force is $5 \%$ of the initial maximum force. $\mathrm{A}$ is the shoulder and $\mathrm{B}$ is the elbow angle, in degrees, and $\mathrm{C}$ and $\mathrm{D}$ are the errors (absolute difference between desired and output angles) for the shoulder and elbow respectively. E shows the muscle activations calculated by the FF+FB controller. 


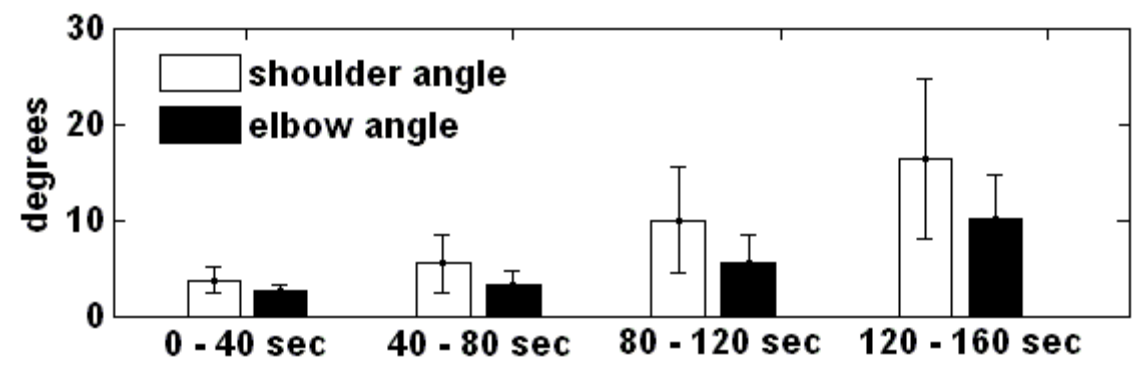

Figure 4-7: The mean RMS error across fifty 160-second trials with fatigue.

Figure 4-8 shows an example of the controller performance in the presence of two sets of random external forces at the hand: small forces (left panels) and large forces (right panels). The forces are shown in $\mathrm{C}$ and $\mathrm{F}$ respectively. A and $\mathrm{D}$ show the shoulder angles, and $\mathrm{B}$ and $\mathrm{E}$ show the elbow angles. The RMS errors for small external forces were 3.8 and 3.7 degrees for the shoulder and elbow angles respectively. The RMS errors for large external force were 6.0 and 4.4 degrees for the shoulder and elbow angles respectively. Note that the RMS errors for the shoulder and elbow for the same trial with no external forces were 2.7 and 2.5 degrees respectively.

The mean RMS error for the shoulder and elbow angle, for all the trials with external forces at the hand are shown in figure 4-9. The error increases with the force amplitude, but in all cases it is below 10 degrees. 

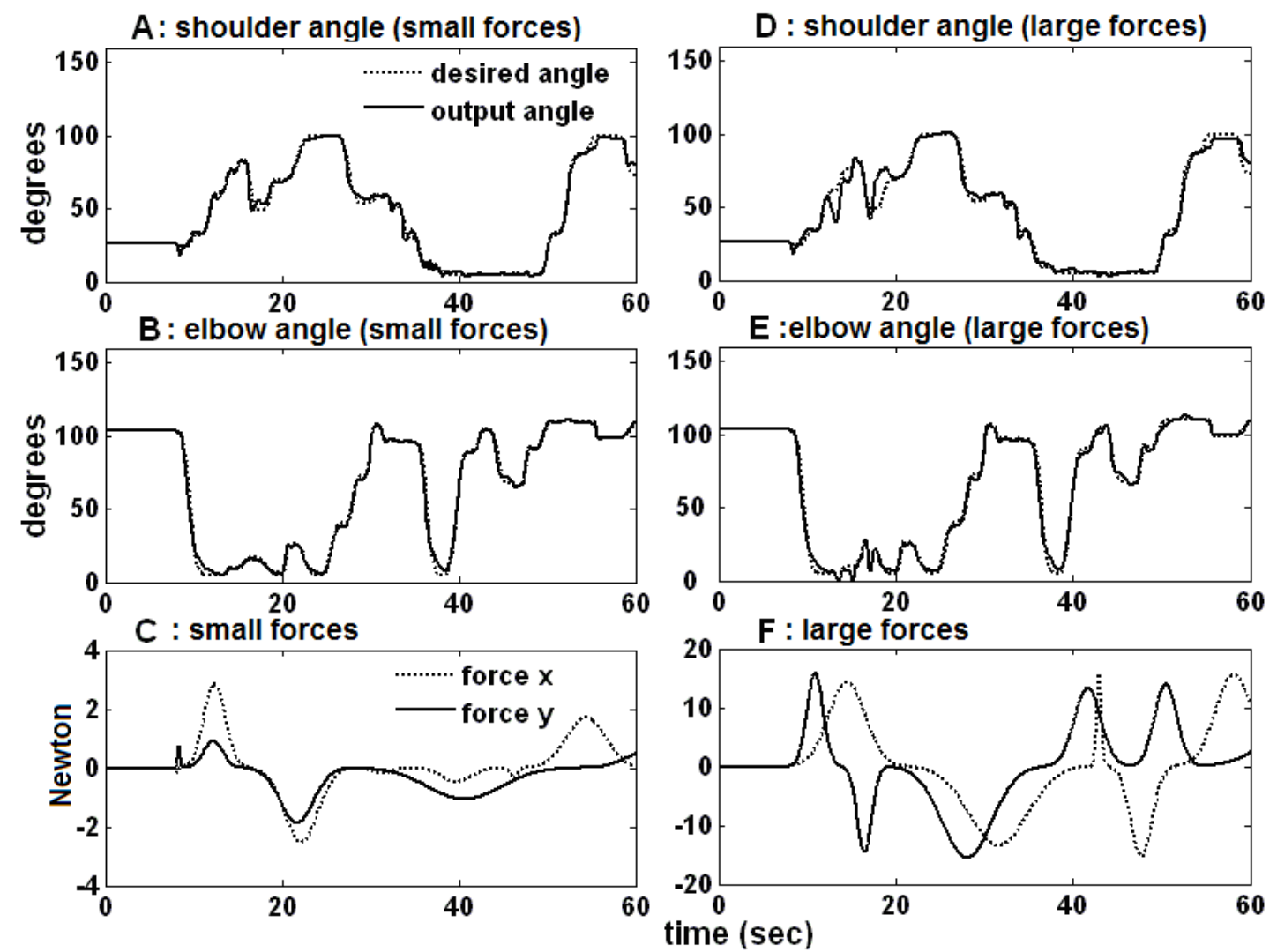

Figure 4-8: Example of the FF+FB controller performance in the presence of two sets of random external forces at the hand: small forces (left panels) and large forces (right panels). The small forces are shown in panel $\mathrm{C}$ and the large forces are shown in panel $\mathrm{F}$ (note the difference in scales). A and D are the shoulder angles, and B and $\mathrm{E}$ are the elbow angles.

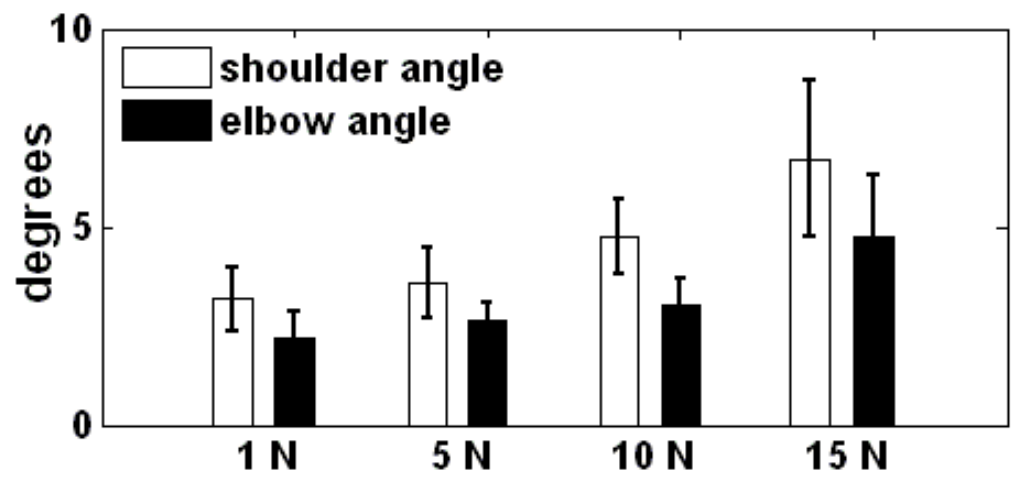

Figure 4-9: The mean RMS error for the shoulder and elbow angle, forty 60 -second trials with external forces at the hand. 


\section{Discussion}

A feedforward-feedback controller has been developed for a two-joint, six-muscle arm model. The inverse-dynamic model was used to train the feedforward ANN, so that the feedforward component takes into account the complex interactions between muscles and joints, and solves the redundancy problem by distributing the muscle forces according to a minimum energy consumption criterion. The inverse-dynamic model in the steady-state is used to train the ANN in the feedback loop, to create a neuro-PD feedback controller capable of handling the highly nonlinear nature of the musculoskeletal system. The controller shows excellent tracking performance with less than 4 degrees error in ideal conditions, and less than 10 degrees even in the case of considerable fatigue and external disturbances.

Using the actual inverse-dynamic model for ANN training solved the redundancy problem caused by the unequal number of joints and muscles in the model. In addition, the muscle activations calculated by the controller were kept at low levels (except for the case of extreme fatigue), since the criterion used to select the unique solution of the muscle force distribution minimizes energy consumption. As shown in figures 4-4 and 4-6, all muscle activations stay mostly below 0.5 , until fatigue increases above $40-50 \%$.

In the comparison among $\mathrm{FF}, \mathrm{FB}$ and $\mathrm{FF}+\mathrm{FB}, \mathrm{FF}$ has the worst performance. This is because the ANN that was trained according to the inverse-dynamic model is not the exact inverse of the forward model. First, the inverse model itself is different than the forward model, due to numerical errors, and the complexity of the muscle model, that cannot be perfectly inverted (Happee 1994). Practical solution of the redundancy problem by optimization requires the use of discrete time steps in the inverse-dynamic model, meaning the time-history dependence of muscle force is not captured as it is in the forward-dynamic 
model (Yoshida et al. 2002). And second, after training, the ANN is still only an approximation of the inverse-dynamic model. In practice, feedforward control will not be used on its own, since it is very difficult to measure all the parameters needed for a perfect inverse-dynamic model of the real human arm (van der Helm 1994). However, as shown in figures 4-3 and 4-5, even an imperfect feedforward component can improve the performance of the overall controller substantially.

Addition of the FF is beneficial in one more way. FB has a tracking performance almost as good as $\mathrm{FF}+\mathrm{FB}$, but it produces muscle activations that are much more active than FF. For example, around the $4^{\text {th }}$ second of the movement shown in figures 4-3 and 4-4, the activation of the anterior deltoid calculated by FB rapidly approaches the maximum. Addition of the FF component smoothes the activation and does not increase it above 0.7.

The fatigue model is very simplistic, since it does not include activation level and stimulation frequency dependence, or a recovery component (Riener et al. 1996). However, our goal was to test the controller in the case of reduced muscle output, and the fatigue model we chose created the right conditions for that. As shown in figures 4-6 and 4-7, under moderate fatigue conditions the tracking performance of the controller is as good as the ideal case. During normal goal-directed movements, it is very unlikely that all the muscles will fatigue below $50 \%$, without a chance for recovery.

The external forces added to the hand simulate obstacles, or objects that are picked up and held during the movement. They have a much lower effect on the controller performance than fatigue: the error is below 10 degrees for forces up to $15 \mathrm{~N}$.

The controller was tested in a two-joint, six-muscle system, but it can easily be implemented in a full arm model. If the inverse-dynamic model is available, the ANN can be 
trained with more inputs and outputs, although a larger number of neurons in the hidden layer may be needed. One important practical benefit of using PD in series with an ANN instead of a purely PD feedback loop is that the number of gains that need to be tuned is reduced to four in the case of our model (proportional and derivative for each angle) instead of 24 (the previous four for each of the six muscles). This becomes a greater advantage when the number of muscles increases.

When the controller is implemented in the real FES system, it is unlikely that the model-based ANN parameters will result in sufficiently good performance, since, as mentioned earlier, it is very difficult to build a perfect inverse-dynamic model of the FES user's arm. In that case, adaptation of the ANN will be necessary, using data collected from the FES-driven arm, as shown in Abbas and Chizeck (1995). Before the controller is tested in the FES system, ANN adaptation techniques will be tested in simulation, to ensure the controller is robust during the adaptation period. 


\title{
Chapter 5: A shoulder and arm controller for trajectory tracking and stability
}

\begin{abstract}
A combined feedforward and feedback controller for the muscles of the upper limb has been developed. It can be used both in simulation, and as part of an actual neuroprosthetic system that stimulates paralyzed muscles to restore upper extremity function. The role of the controller is to calculate the required muscle activations not only to allow the arm to follow a desired trajectory, but also to ensure glenohumeral stability. In order to achieve this goal, it consists of two levels: the first includes an artificial neural network trained according to the inverse dynamics of the arm, combined with a variable-gain PD controller, and its role is to allow for tracking of the desired trajectory. The second level is an open-loop controller that uses the outputs of the previous level to adjust the activations of the rotator cuff muscles and thus ensure glenohumeral stability. The performance of the controller is demonstrated in a 3-dimensional biomechanical model of the arm that includes 5 degrees of freedom, 22 muscles, and realistic arm dynamics.
\end{abstract}

\section{Introduction}

A neuroprosthesis is a system that applies functional electrical stimulation (FES) to paralyzed muscles, to restore function to individuals with spinal cord injury. The controller for this system calculates the required muscle stimulation levels, based on the user commands. In the case of a high level upper extremity neuroprosthesis, which targets individuals with no voluntary function below the neck, the controller needs to activate the appropriate shoulder and arm muscles to allow coordinated arm movements to be performed. 
This is a complicated task, due to the large number, redundancy and highly non-linear nature of the muscles, the biomechanical interaction between muscles and joints, and the extreme variety of goal-oriented arm movements as opposed to the cyclic patterns seen in the lower extremity (Crago et al. 1996).

Another challenge for control in high tetraplegia is that the large range of arm motion is achieved at the cost of glenohumeral stability (Veeger and van der Helm 2007). The glenohumeral (GH) joint is a ball-and-socket joint, and translation is avoided by compression of the humeral head against the glenoid. This is mainly achieved through the action of the rotator cuff muscles (supraspinatus, infraspinatus, subscapularis and teres minor), which are positioned in a half-circle around the GH joint, and therefore are particularly suitable for pulling the humerus into the glenoid. When these muscles are paralyzed, it is the responsibility of the FES controller to appropriately stimulate them in order to ensure GH stability. This task is made harder by the fact that it is not currently possible to measure the resulting force on the glenoid of the FES user, and therefore predict possible GH instability.

Current FES systems for restoration of upper extremity motor function have focused on hand grasp (Handa and Hoshimiya 1987; Peckham et al. 1988; Popovic et al. 1999), elbow flexion-extension (Crago et al. 1998) and pronation-supination (Lemay et al. 1996) of individuals with mid-level tetraplegia. Hoshimiya et al. described a percutaneous system for C4 level tetraplegia, which targeted the shoulder, elbow, wrist and fingers, using preprogrammed stimulation patterns to restore a very limited number of movements (Hoshimiya et al. 1989). The shoulder patterns were based on EMG measurements from able-bodied individuals (Kameyama et al. 1999), and did not take into account the fact that in paralyzed subjects all muscles are not necessarily available for stimulation, due to denervation. In order 
to restore glenohumeral stability, but not shoulder motion, stimulation of the supraspinatus, deltoid and trapezius has been applied in individuals with hemiplegia, successfully reducing shoulder subluxation (Yu et al. 2004).

In order to cope with the new challenges of shoulder mobility and stability, our controller uses a two-level design: the first level consists of a feedforward-feedback controller based on non-linear PD and artificial neural networks (ANN), responsible for tracking the desired trajectory; the second level is an open-loop controller that monitors the outputs of the feedforward and feedback components, and adjusts the activations of the rotator cuff muscles to ensure glenohumeral stability. The controller design is tested in a large-scale model of the upper extremity that includes 22 muscles, three degrees of freedom at the glenohumeral joint, elbow flexion-extension and pronation-supination. The trajectory tracking performance of the controller is tested using realistic goal-oriented movements. Its ability to maintain glenohumeral stability is tested in the presence of external forces acting on the elbow and hand.

\section{Methods}

\section{The model}

The controller was developed and tested in a 3-dimensional model of the upper limb, that includes five degrees of freedom (three at the glenohumeral joint, elbow flexionextension and forearm pronation-supination), and 22 muscles, divided into 102 elements according to their lines of action. The muscle and joint parameters were taken from cadaver studies performed by Klein-Breteler et al. (1999). Wrapping objects based on geometrical structures allowed for realistic calculation of the muscle lines of action and moment arms. 
The muscle model is a Hill-type model with two states (activation and muscle fiber length) and three elements (contractile, series elastic and parallel elastic) developed by McLean et al. (2003).

The shoulder joint cannot dislocate in the model, as the joint is modelled as a balland-socket joint in which translation is not permitted, but glenohumeral stability is measured using the "glenohumeral stability index", calculated from the force vector on the glenoid resulting from all the muscle forces acting on it, and potential external forces on the arm. It is defined as:

$G H_{s t a b}=\left(\frac{\theta}{\theta_{a}}\right)^{2}+\left(\frac{\varphi}{\varphi_{a}}\right)^{2}$

where the glenoid is approximated by an ellipse, $\theta$ and $\varphi$ are the angles of the force vector along the axes of the ellipse away from normal to the glenoid, and $\theta_{a}$ and $\varphi_{a}$ are the corresponding angles at the rim of the glenoid. As long as $\mathrm{GH}_{\text {stab }}<1$, the force vector points inside the glenoid fossa and the joint is stable.

When the model is used as a substitute for the human arm, it runs in a forward dynamic way, where the inputs are the muscle activations and potential external forces, and the outputs are the joint angles and the glenohumeral stability index. However, the model can also run inverse dynamic simulations, which is used for controller training. In this case, the inputs are the desired joint angles and possible external forces, and the outputs are the required muscle activations. Since there are more muscles than degrees of freedom, the muscle activations are calculated using an optimization routine that takes into account the glenohumeral stability constraint, and minimizes the sum of squares of the muscle activations: 
$\min \sum_{i=1}^{22} a c t_{i}^{2}$

Note that each of the 102 muscle elements included in the model is not activated separately. In order to simulate a neuroprosthetic system, the controller is constrained to apply the same level of activation to all elements in a muscle, similar to a muscle or nerve electrode of the

real system. Since the inverse dynamic model is used to train the controller, there are only 22 independent muscle activations calculated by the optimization routine.

\section{The controller}

The controller design is shown in figure 5-1. It consists of a feedforward and a feedback part, which calculate activations independently and add them, and subsequently a controller for glenohumeral stability. The input to the controller is the desired arm trajectory, expressed in joint angles. These were chosen according to the protocol described by van der Helm (1997): humeral plane of elevation (GHy), humeral elevation $(\mathrm{GHz})$, humeral rotation (GHyy), elbow flexion-extension (ELx) and forearm pronation-supination (PSy). The feedforward part is an ANN trained using data from inverse dynamic simulations. It is a twolayer network with a sigmoidal transfer function in the hidden layer, and a linear transfer function in the output layer. It has ten inputs: five angles and five angular velocities, 20 neurons in the hidden layer, and 22 outputs (the muscle activations). Its role is to model the arm dynamics, and provide an initial estimate of the muscle activations needed to perform a desired movement. Since an FES user is expected to perform slow movements, a second ANN was trained with steady-state data, in order to test the importance of including dynamics in the feedforward controller. It has the same architecture as the dynamic ANN, with fewer inputs (five angles at the steady-state) and 15 neurons in the hidden layer. The 
movements used for training were goal-oriented movements inside the workspace allowed by the five degrees of freedom; since the model does not include a moving scapula, this workspace is limited compared to that of an arm with a complete shoulder description.

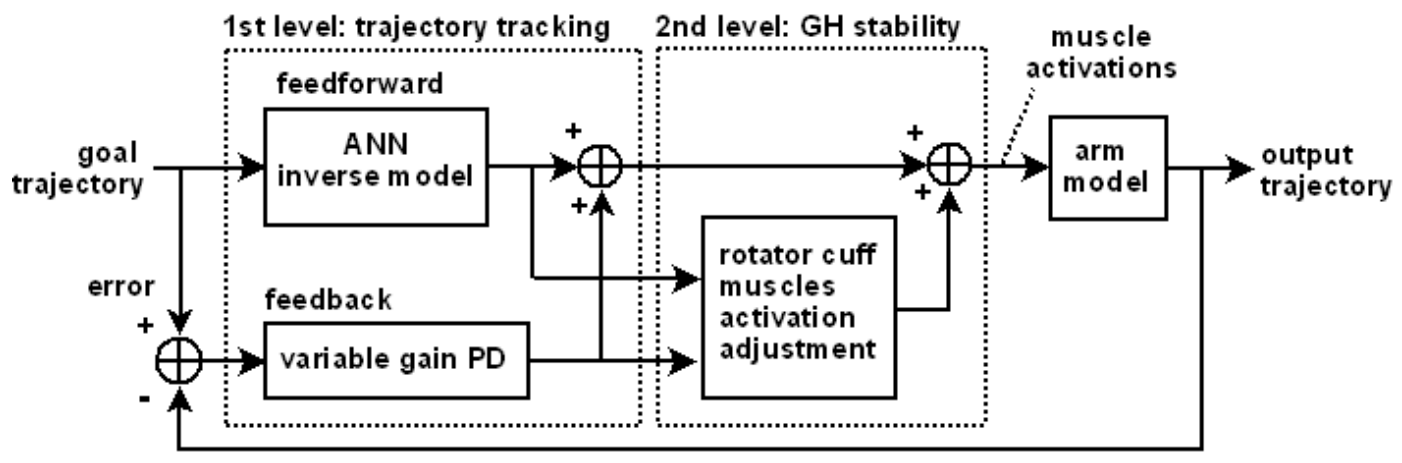

Figure 5-1: The controller design

The feedback controller corrects for inaccuracies in the inverse dynamics modelled by the feedforward ANN, and also external disturbances (e.g. the weight of an object picked up by the hand). It was designed as a PD controller with variable gains. The integral term was not included because it caused instability. The gains depend on the muscles' moment arms at every position, but since the actual values of the moment arms are assumed to be unknown in a real FES user, only the signs of the moment arms are used. For some muscles, particularly the ones crossing the elbow, the moment arm has the same sign in all positions (e.g. the brachialis moment arm about the elbow joint is always positive). However, for muscles crossing the glenohumeral joint which has three axes of rotation, some moment arms change sign. For example, the middle fibers of the supraspinatus muscle tend to externally rotate the humerus when the arm is internally rotated, and internally rotate the humerus when the arm is externally rotated. Since the exact position of change in the moment arm might be 
different between the model and the actual human arm, when the moment arm is below a threshold, it is assumed to have no effect (positive or negative) on the joint. This threshold was set to $0.5 \mathrm{~mm}$, chosen as a small value compared to the maximum muscle moment arms in the model, which range from $6 \mathrm{~mm}$ (anconeus) to $90 \mathrm{~mm}$ (brachioradialis).

The activation adjustment for every muscle is calculated according to the following equation:

$$
\operatorname{act}_{i}(t)=\sum_{j=1}^{5}\left[\left(K_{P j} e(t)+K_{D j} \frac{d e(t)}{d t}\right) \cdot f\left(\operatorname{momarm}_{i j}(t)\right)\right], \quad \begin{array}{r}
1, \text { if }^{\operatorname{momarm}_{i j}}(t) \geq 0.5 \mathrm{~mm} \\
\left.\quad \operatorname{momarm}_{i j}(t)\right)= \\
0, \text { if }\left|\operatorname{momarm}_{i j}(t)\right|<0.5 \mathrm{~mm} \\
-1, \text { if } \operatorname{momarm}_{i j}(t) \leq-0.5 \mathrm{~mm}
\end{array}
$$

where momarm $\mathrm{ij}_{\mathrm{j}}(\mathrm{t})$ is the moment arm of muscle $i$ about degree of freedom $j$ at time t. The (constant) $P D$ coefficients $K_{P \mathrm{j}}$ and $\mathrm{K}_{\mathrm{Dj}}, \mathrm{j}=1 \ldots 5$ are shown in table 5-1.

\begin{tabular}{|c|c|c|c|c|c|}
\hline & GHy & GHz & GHyy & ELx & PSy \\
\hline $\mathrm{K}_{\mathrm{P}}$ & 60 & 60 & 60 & 10 & 70 \\
\hline $\mathrm{K}_{\mathrm{D}}$ & 50 & 50 & 50 & 5 & 80 \\
\hline
\end{tabular}

Table 5-1: The PD coefficients of the feedback controller, which multiply the moment arm based gains to calculate the activation adjustment for each muscle

The controller for GH stability regulates the activation of the rotator cuff muscles (infraspinatus, supraspinatus, subscapularis and teres minor), whose main action is not to move, but to stabilize the GH joint. It is not possible for the controller to know when GH stability is compromised, since the resulting force on the glenoid is assumed to be unknown. (The "GH stability index" is an output of the model, but it cannot be measured in the real FES user.) However, if the muscle activations driving the arm are mostly feedforward, GH stability is guaranteed by the fact that the feedforward controller was trained with data from inverse dynamic simulations that included the GH stability constraint. Thus, the controller 
monitors the contributions of the feedforward and feedback components, and increases the activation of the rotator cuff muscles when the feedback activations increase:

$a c t_{\text {RotatorCuff }}=a c t_{\text {RotatorCuff }} \bullet\left(1+\frac{\sum a c t_{F B}}{\sum a c t_{F F F B}}\right)$

where $\sum a c t_{F B}$ and $\sum a c t_{F F F B}$ include all muscles except for the rotator cuff.

This is an indication of an external disturbance that might cause instability, and activating the rotator cuff muscles protects the joint, without notably affecting the desired arm trajectory.

\section{Controller evaluation}

The controller was tested using goal-oriented movements, similar but not identical to the ones used for controller training. The error in the trajectory tracking was quantified using the following two error measures at the endpoint, which in this model is the wrist (midpoint of the ulnar and radial styloids):

endpoint position error: the distance between the desired and actual endpoint

endpoint orientation error: the angle between the desired and actual endpoint orientation.

This is calculated by extracting the rotation angle (about an arbitrary axis) from the rotation matrix between the desired and actual forearm coordinate frames.

For a rotation of angle $\theta$ about the axis $\mathbf{n}=\left[\mathrm{n}_{\mathrm{x}} \mathrm{n}_{\mathrm{y}} \mathrm{n}_{\mathrm{z}}\right]$, the rotation matrix is:

$$
R=\left[\begin{array}{ccc}
n_{x}^{2}+\left(n_{y}^{2}+n_{z}^{2}\right) \cos \theta & n_{x} n_{y}(1-\cos \theta)-n_{z} \sin \theta & n_{x} n_{z}(1-\cos \theta)+n_{y} \sin \theta \\
n_{x} n_{y}(1-\cos \theta)+n_{z} \sin \theta & n_{y}^{2}+\left(n_{x}^{2}+n_{z}^{2}\right) \cos \theta & n_{y} n_{z}(1-\cos \theta)-n_{x} \sin \theta \\
n_{x} n_{z}(1-\cos \theta)-n_{y} \sin \theta & n_{y} n_{z}(1-\cos \theta)+n_{x} \sin \theta & n_{z}^{2}+\left(n_{x}^{2}+n_{y}^{2}\right) \cos \theta
\end{array}\right]
$$

The root mean square position and orientation error:

$$
R M S E=\sqrt{\frac{\sum_{i=1}^{N}(\text { error })^{2}}{N}}
$$


was used to quantify the tracking performance for the duration of a trial. However, since functionally the important part of a goal-oriented movement is not the trajectory, but the goal position and, if part of the movement, the return to the initial position, the "goal" and "return" errors were calculated separately for each trial. The GH stability was measured using the value of the GH stability index. For shoulder stability to be maintained, the index had to stay below 1 for the entire duration of all trials.

In the previous simulations, since the feedforward controller is not a perfect inverse model, there was always a small amount of feedback required, which resulted in a small increase of the rotator cuff muscle activations. In order to test whether GH stability is maintained when large feedback activations are needed, external forces in different directions ranging from 10 to $30 \mathrm{~N}$ and lasting from 1 to 5 seconds were applied either at the hand or the elbow. The arm was either kept at a constant position, or made to follow a goal-oriented trajectory similar to the ones described before. The same simulations were performed with and without the rotator cuff muscles activation adjustment, and the GH stability index was calculated in each case.

\section{Results}

Figure 5-2 shows an example of a goal-oriented movement, controlled by the static feedforward controller only. The first two panels show the goal and actual angles, divided into the three shoulder angles (A), and elbow-forearm angles (B). Panel C shows the endpoint position error (in $\mathrm{cm}$ ), and D shows the endpoint orientation error (in degrees). The eight highest muscle activations are shown in panel E, and the GH stability index is shown in panel F. 

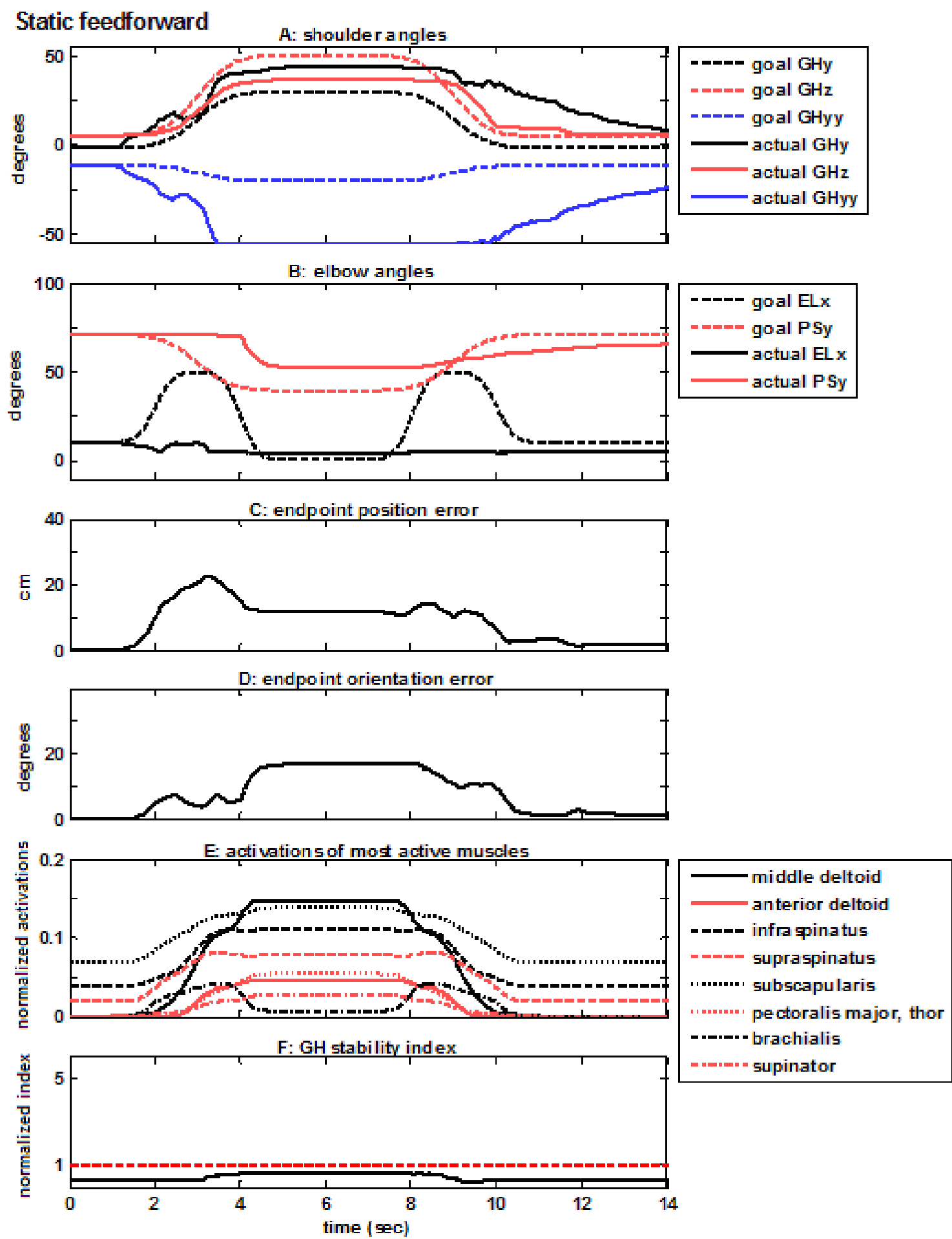

rachialis

Figure 5-2: Example of a goal-oriented movement using the static feedforward controller. Panel A shows the shoulder angles, in dashed lines for the goal, and solid lines for the actual. Panel B shows the elbow and forearm angles. $\mathrm{C}$ is the endpoint position error (in $\mathrm{cm}$ ), and $\mathrm{D}$ is the endpoint orientation error (in degrees). Panel E shows the activations of the eight muscles that were most active during this movement. Finally, panel F shows the GH stability index (solid dark line). The light-colored dashed line indicates the limit for stability (index=1). 
The same movement controlled by the dynamic feedback controller only, is shown in figure $5-3$.

Dynamic Feedforward

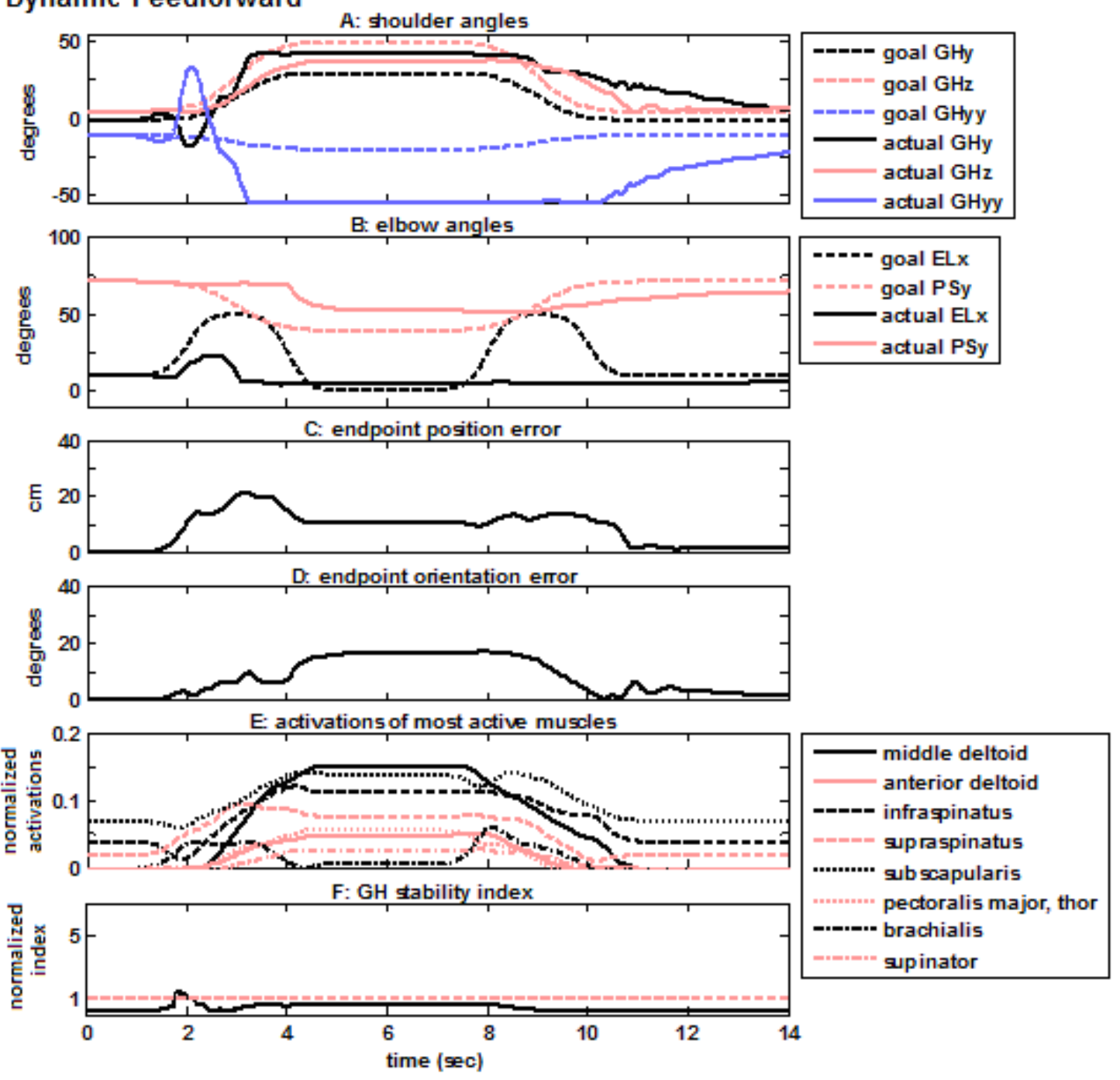

Figure 5-3: The same movement using the dynamic feedforward controller. Like the previous figure, panel A shows the shoulder angles, in dashed lines for the goal, and solid lines for the actual. Panel B shows the elbow and forearm angles. C is the endpoint position error (in $\mathrm{cm}$ ), and $\mathrm{D}$ is the endpoint orientation error (in degrees). Panel $\mathrm{E}$ shows the activations of the eight muscles that were most active during this movement. Finally, panel F shows the GH stability index (solid dark line). The light-colored dashed line indicates the limit for stability (index=1). 
The same movement is controlled by the feedback controller only, in figure 5-4.

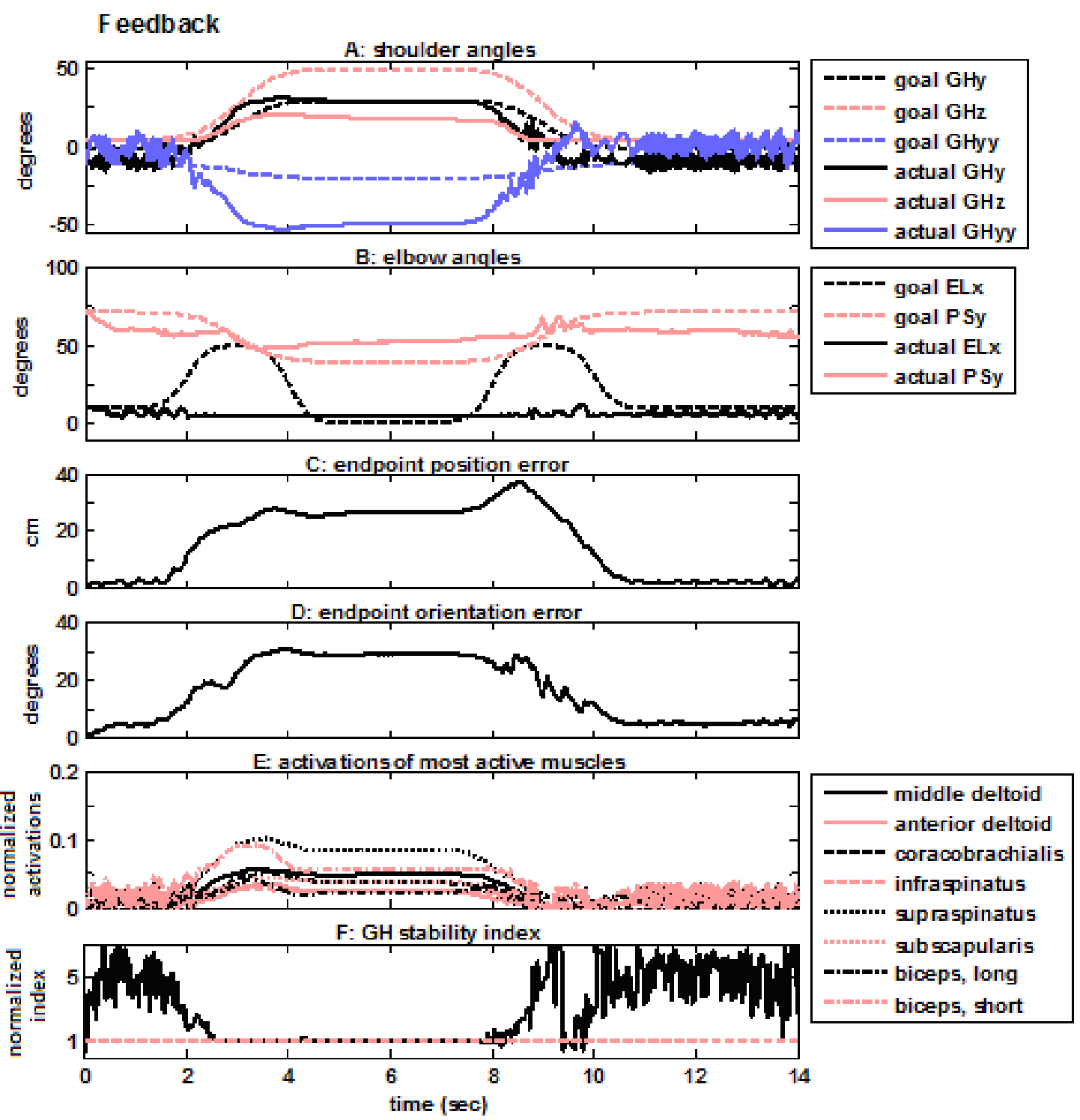

Figure 5-4: The same example using only the feedback controller. Like the previous figures, panel A shows the shoulder angles, and B shows the elbow and forearm angles (in dashed lines for the goal, and solid lines for the actual). $\mathrm{C}$ is the endpoint position error (in $\mathrm{cm}$ ), and D is the endpoint orientation error (in degrees). Panel $\mathrm{E}$ shows the activations of the eight muscles that were most active during this movement, and panel F shows the GH stability index (solid dark line). The light-colored dashed line indicates the limit for stability (index=1). 
Finally, the combined dynamic feedforward-feedback controller is applied to the movement, shown in figure 5-5.

\section{Dynamic feedforward - feedback}
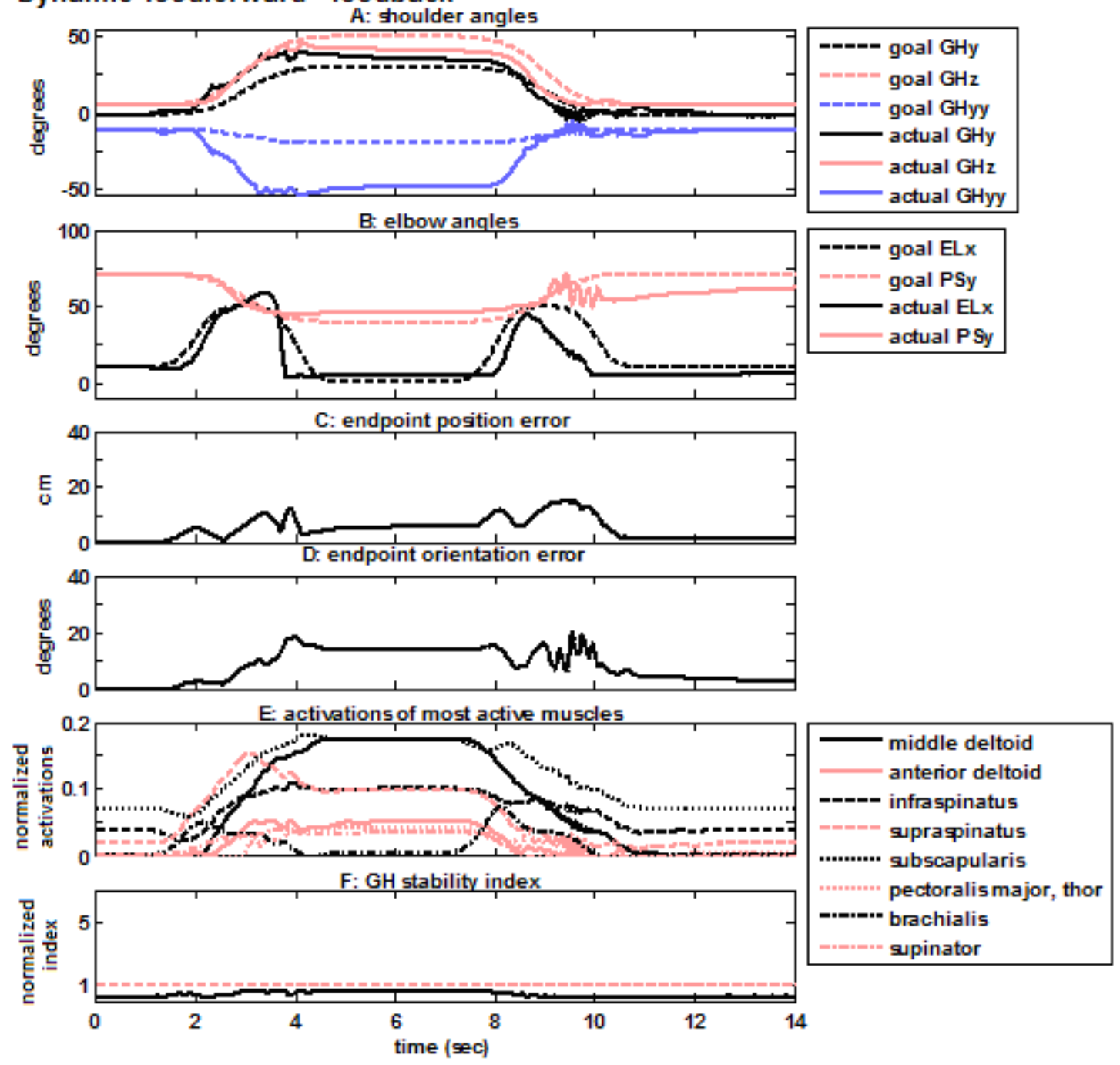

Figure 5-5: The same example using the combined dynamic feedforward-feedback controller. Like the previous figures, panel A shows the shoulder angles, and B shows the elbow and forearm angles (in dashed lines for the goal, and solid lines for the actual). $\mathrm{C}$ is the endpoint position error (in $\mathrm{cm}$ ), and $\mathrm{D}$ is the endpoint orientation error (in degrees). Panel $\mathrm{E}$ shows the activations of the eight muscles that were most active during this movement, and panel $\mathrm{F}$ shows the GH stability index (solid dark line). The light-colored dashed line indicates the limit for stability (index=1). 
Figure 5-6 summarizes the differences in the performance of the static and dynamic feedforward controllers. The mean endpoint position (panel A) and orientation error (panel B) are calculated from fifty 14-second goal-oriented movements, similar to the one shown before, and the two controllers have a similar performance (no significant difference, $\mathrm{p}>0.1$ ).
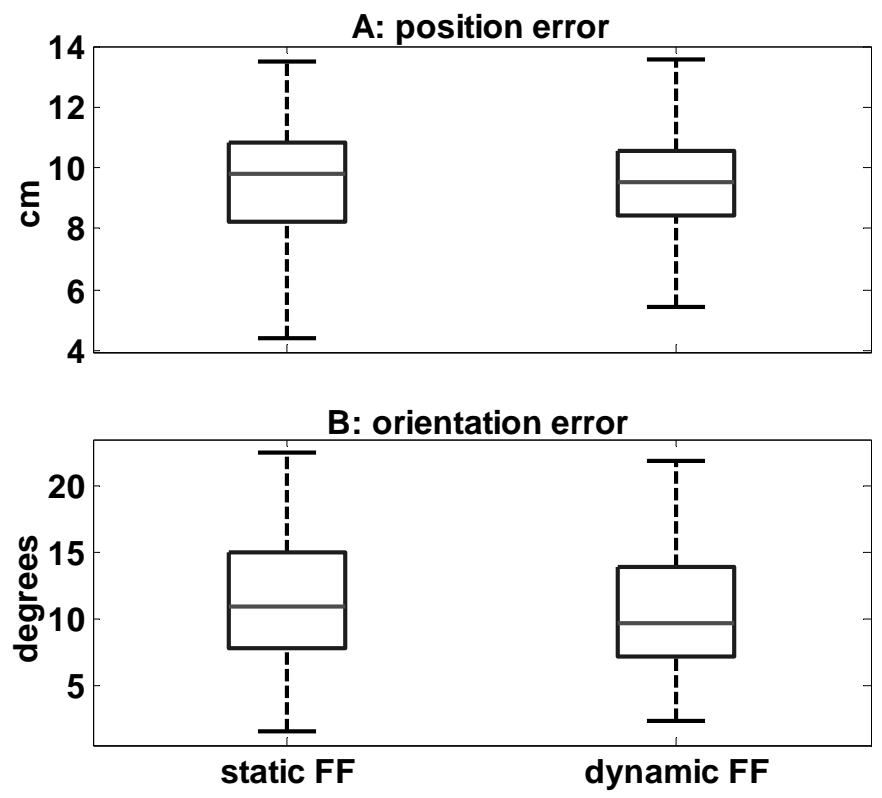

Figure 5-6: Comparison of the performance of the static (left boxplot) and dynamic (right boxplot) feedforward controllers in fifty 14-second goal-oriented movements. Panel A shows the endpoint position error, and panel $\mathrm{B}$ the endpoint orientation error. The errors between the two controllers are not significantly different $(\mathrm{p}>0.1)$. 
Figure 5-7 shows the position and orientation errors from the same fifty 14 -second goal-oriented movements, using the combined feedforward-feedback controller. The errors are calculated during the entire movement (left), at the "goal" of the movement only (middle), and at the end of the movement only, when the arm returns to the initial position (right).
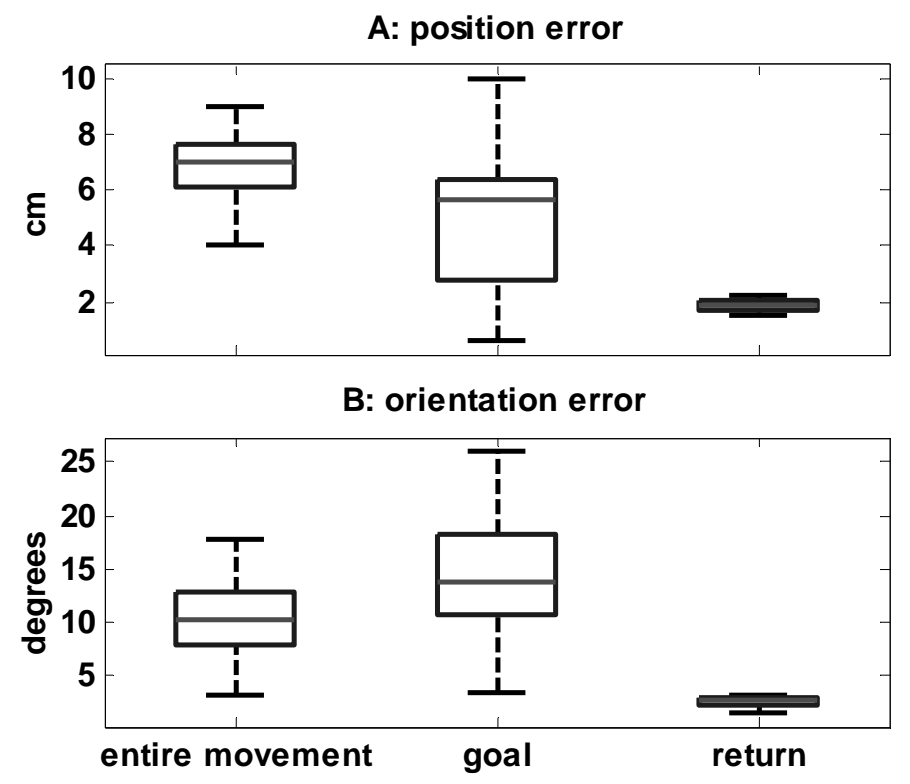

Figure 5-7: Summary of errors from fifty 14-second goaloriented movements using the combined feedforward-feedback controller. Panel A shows the position error, and panel B the orientation error. The left boxplot is the error during the entire movement, the middle is the error at the goal of the movement, and the right is the error at the return to the initial position. 
The GH stability index for the same fifty trials using the combined feedforwardfeedback controller is shown in figure 5-8. It stays below 1 for the entire duration of all trials, which means that GH stability is always maintained.

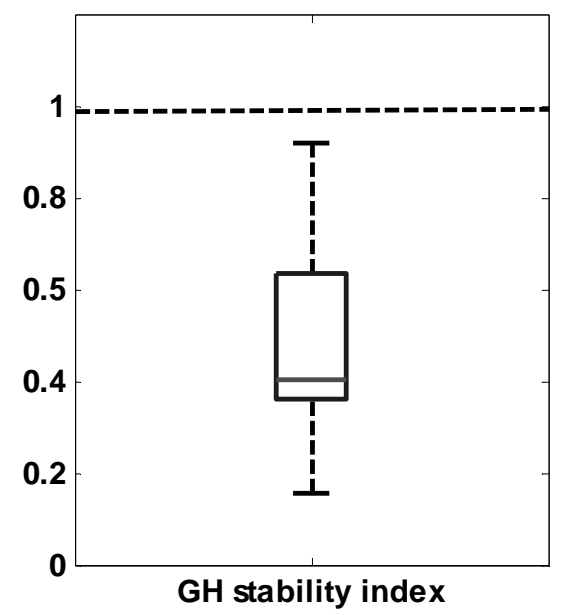

Figure 5-8: The GH stability index for fifty goal-oriented movements using the feedforward-feedback controller.

Figure 5-9 shows an example of a trial with an external force of $20 \mathrm{~N}$ applied to the elbow for 2 seconds, while the input angles were held constant. This trial did not include GH stability correction. Panel A shows the external force, and the rest of the panels are the same as the previous examples. 

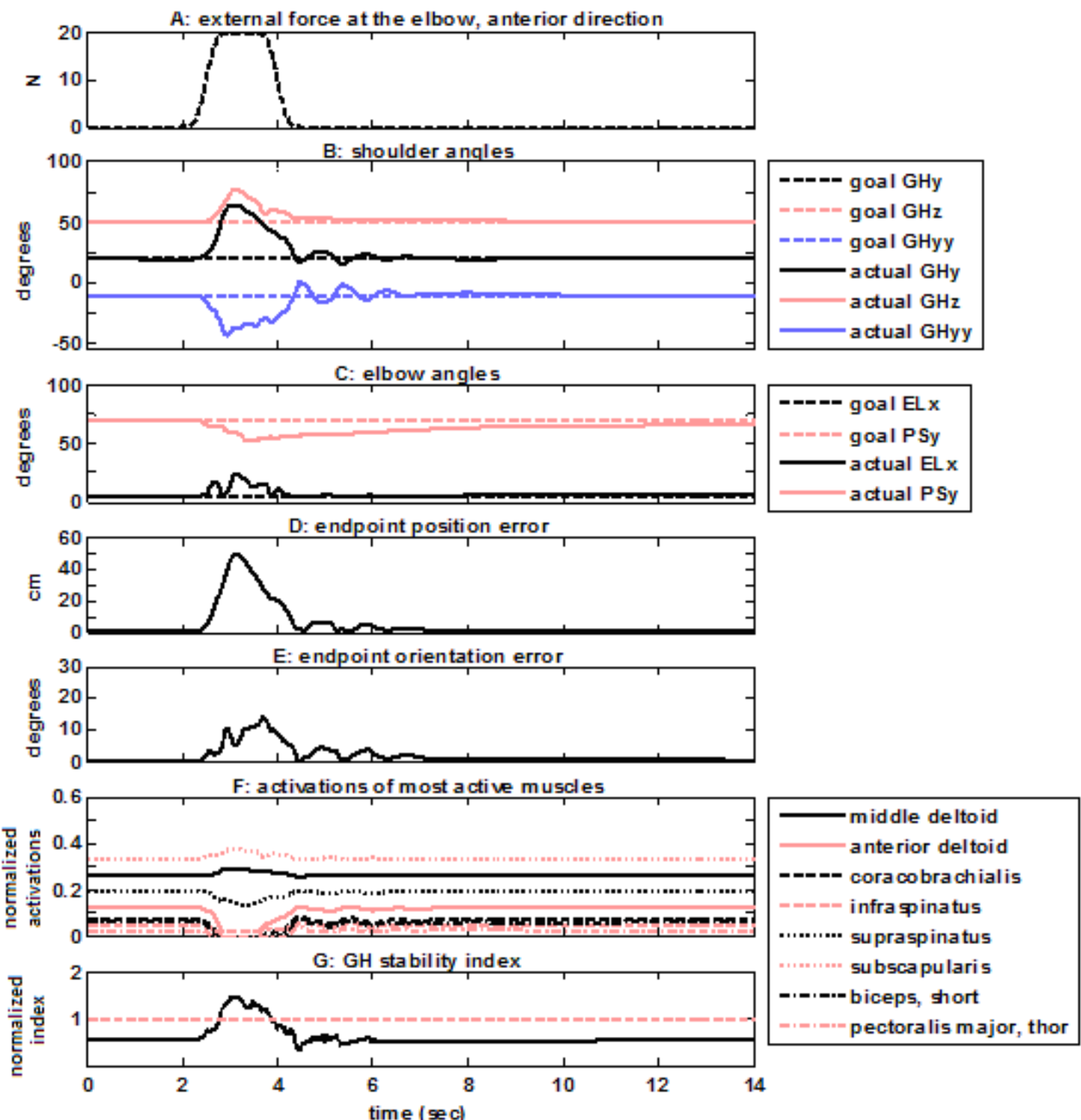

Figure 5-9: Example of the application of an external force without GH stability correction. The force, applied at the elbow in the anterior direction is shown in panel A. Panel B shows the shoulder angles, and $\mathrm{C}$ shows the elbow and forearm angles. $\mathrm{D}$ is the endpoint position error, and $\mathrm{E}$ is the endpoint orientation error. Panel $\mathrm{F}$ shows the activations of the eight muscles that were most active during the movement, and panel G shows the GH stability index (solid dark line). The light-colored dashed line indicates the limit for stability (index=1). 
Figure 5-10 shows the same example including GH stability correction.
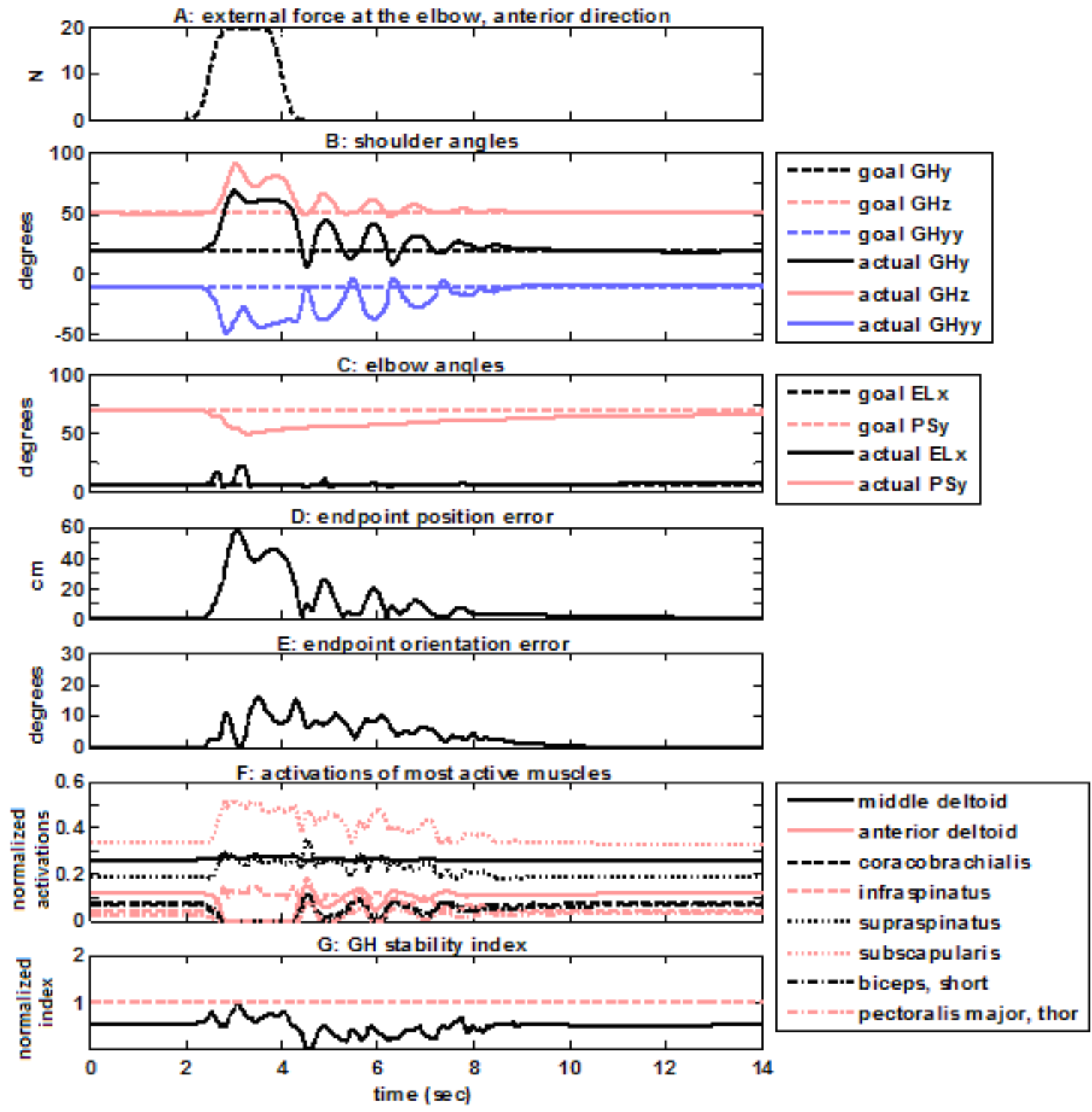

Figure 5-10: The same example with GH stability correction. As before, the force is shown in panel A. Panel B shows the shoulder angles, and C shows the elbow and forearm angles. D is the endpoint position error, and $\mathrm{E}$ is the endpoint orientation error. Panel $\mathrm{F}$ shows the activations of the eight muscles that were most active during the movement, and panel $G$ shows the GH stability index. 
Finally, Figure 5-11 shows the GH stability index from thirty trials that either included or not the controller for rotator cuff muscle activation adjustment.

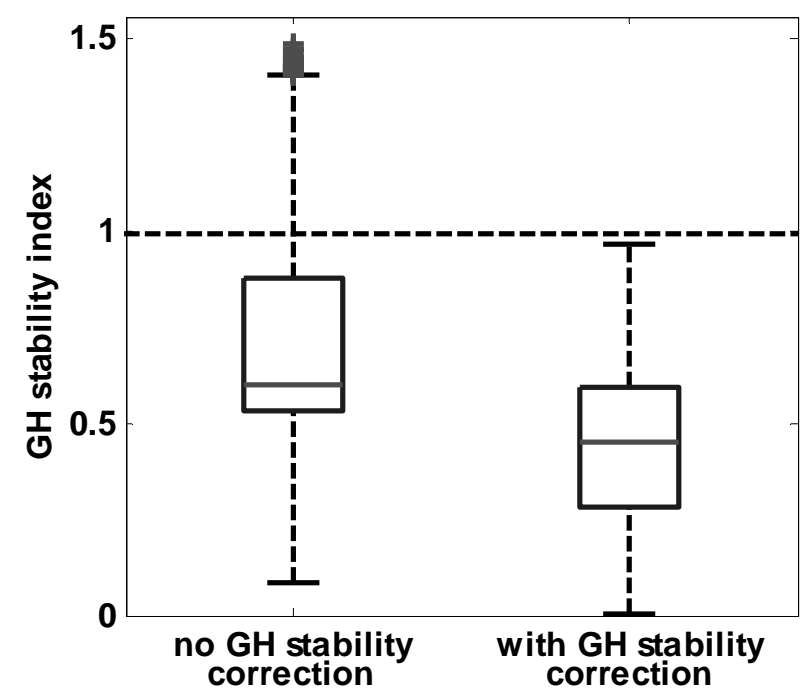

Figure 5-11: The GH stability index for all trials with (right boxplot) and without (left boxplot) the stability correction.

\section{Discussion}

A controller for the entire upper extremity has been developed, which combines tracking of the desired trajectory, with stability of the glenohumeral joint. The controller was tested in a large-scale biomechanical model that has a fixed scapula, but includes all the muscles that cross the $\mathrm{GH}$ joint and elbow. The endpoint error was below $10 \mathrm{~cm}$ and 25 degrees for the duration of all the movements tested. GH stability was maintained, even in the presence of $30 \mathrm{~N}$ external forces.

The static and dynamic feedforward controllers have a similar performance, as shown in figures 5-2, 5-3 and 5-6. This suggests that, if we are interested in slow movements only, it is not necessary to include the angular velocities of the joints as inputs to the feedforward 
controller. This would result in a smaller ANN with fewer weights, which would require less training. However, collecting steady-state data would be more time-consuming than dynamic data, which is not a limitation in simulation, but it could be inconvenient for the actual FES user. For this practical reason, as well as to avoid movement velocity limitations, the dynamic controller was preferred for the combined feedforward-feedback design.

When the feedforward controller was applied by itself, the calculated activations did not produce the desired movement, as seen in both the static and dynamic feedforward controller examples in figures 5-2 and 5-3. This is because even though the feedforward controller is trained according to the inverse dynamic model, it is not a perfect inverse of the forward dynamic model (it does not accurately capture the time-dependence of muscle force), and in addition, the ANN is only an approximation of the inverse dynamic model. Due to the errors in the resulting kinematics, GH instability might occur (e.g. large internal rotation of the humerus (GHyy) around second 2 in the movement shown in figure 5-3 produces different than expected muscle lengths, and consequently muscle forces, which causes instability). On the other hand, the feedback controller by itself did not ensure GH stability, as shown in panel $\mathrm{F}$ of figure 5-4.

Figure 5-5 shows that the combination of feedforward and feedback keeps the arm close to the desired trajectory, and ensures GH stability with slightly elevated rotator cuff activations. The feedforward activations bring the arm close enough to the desired trajectory that the feedback controller can correct without large oscillations. Note that the large errors in humerus plane of elevation (GHy) and humerus rotation angle (GHyy) do not correspond to large errors in the endpoint position and orientation. For example, around second 6, when the arm has reached the "goal" of the movement, the GHyy error is 30 degrees, but the 
endpoint error is only $5 \mathrm{~cm}$ in position and 15 degrees in orientation. This error could be corrected with adjustment of the wrist orientation, which is not included in this model.

The tracking performance might also be improved if fewer muscles were used for control of the system. Redundancy is certainly a desirable property, especially in individuals with paralysis, whose muscles can produce less than half the force of able-bodied people (Kobetic and Marsolais 1994). However, the large number of muscles compared to the degrees of freedom hinders the work of the feedback controller, since it causes a large number of equilibrium states were the muscle activation levels are not zero but produce no movement because they act in opposite directions. In such a case, the error may not be zero, but the activations calculated by the feedback controller have no effect. By testing different muscle sets, it might be possible to find a number of muscles that is still redundant but is easier to control.

When external forces were applied to the elbow or hand, GH instability was not necessarily a problem, since it depended on the direction and the amplitude of the force. However, since GH stability cannot be measured in a current FES system, increasing the rotator cuff muscle activations whenever there is a chance of instability could be a useful safety measure. The exact amount of activation increase would have to be determined based on the shoulder stiffness and muscle strength of the specific FES user.

In conclusion, we believe that the controller design presented in this study is ready to be used in an actual neuroprosthesis for restoration of motor function in high tetraplegia. Its performance was demonstrated in a model that includes 22 muscles of the shoulder and arm, but the same design can be applied in any muscle set that is targeted by the neuroprosthesis, as long as it includes some of the rotator cuff muscles for GH stability. 


\title{
Chapter 6: Adaptive control using co-contraction of antagonistic muscles
}

\begin{abstract}
Functional electrical stimulation (FES) systems aim to restore function to individuals with a wide variety of injury levels, muscle strength, and remaining voluntary control. In this study, an adaptation technique is presented for adjusting the parameters of an FES controller to match the characteristics of a specific subject. The system controlled is the elbow joint, using a pair of antagonistic muscles. The controller is an artificial neural network (ANN) trained on the inverse dynamics of the arm. The input muscle activations and the resulting elbow flexion-extension angle are recorded while the system is in use, and are later used to retrain the ANN. Since there are different combinations of antagonistic muscle activations that can produce the same output angle, a parameter that quantifies the muscle co-contraction has been added to allow for accurate training of the ANN. The adaptation technique is first tested in a biomechanical model of the elbow, and subsequently in one subject who has received an implanted FES system following a high cervical level spinal cord injury.
\end{abstract}

\section{Introduction}

A functional electrical stimulation (FES) system restores motor function after spinal cord injury by stimulating paralyzed muscles. The FES controller calculates the stimulation patterns that need to be applied to the appropriate muscles in order to perform the desired movement. The most commonly used type of control is feedforward, which depends only on the user command, and not the system output. In clinical use, feedforward control usually consists of pre-programmed stimulation patterns (Marsolais and Kobetic 1987; Hoshimiya at 
al. 1989; Peckham et al. 1988), which only allow for a small number of pre-specified movements. Another option, which does not limit the flexibility of motions available to the FES user, is direct inverse control, where the inputs and outputs of the system to be controlled, become the outputs and inputs, respectively, of the feedforward controller (Norgaard et al. 2000). This is sometimes combined with feedback control, which uses sensors to adjust the muscle activations in order to correct for errors in the system trajectory (Crago et al. 1996).

In the implementation of direct inverse control, an artificial neural network (ANN) is usually trained as the inverse model, because it can approximate any dynamic system based solely on the knowledge of inputs and outputs (Norgaard et al. 2000). It is typically trained with data collected from the actual system. For example, Chang et al used data from one able-bodied and one paraplegic subject to train the feedforward part of a controller for the knee joint using stimulation of the quadriceps (Chang et al. 1997). Moving a step further, Riess and Abbas implemented online adaptive control, where the quadriceps stimulation pattern was adjusted during the system use, to improve the performance of a cyclic movement (Riess and Abbas 2000).

Many FES controllers have been developed using computer models in the place of real biomechanical systems (Ferrarin et al. 2001; Jezernik et al. 2004; Pedrocchi et al. 2006). Model-based approaches offer many advantages, as they allow the exploration of interventions and testing of control methods without invasive procedures or time-consuming sessions of trial-and-error. However, the differences between the models and the actual human subjects result in reduced performance of the model-based FES controllers. So in the 
case of controllers developed in simulation, adaptation with data from the actual system is still needed to improve the controller performance.

The controller designs mentioned above are applied to single-joint, single-muscle systems. It is then easy to invert the recorded data and train the feedforward controller. However, when there are more muscles than degrees of freedom, the inverse of the system is not unique, and presented with different activation combinations for the same kinematic configuration, the ANN would train on the average of all possible solutions, which is not necessarily a correct solution (Karniel and Inbar 2000). For example, in the case of two antagonist muscles crossing one joint, there are different activation pairs that can produce the same joint angle. Lujan addressed this problem by using the non-unique data to train an intermediate ANN as a forward model of the system, and then used this model to optimize the data and limit co-contraction of antagonist muscles. The inverse ANN controller was then trained with the optimized, unique data (Lujan 2007). The limitation of this method is that it has multiple steps, including optimization that is typically computationally intensive, and thus it would be difficult to implement in real time.

Our solution to the redundancy problem is to use the co-contraction level of the antagonist muscles to distinguish among all possible solutions. The co-contraction level becomes one of the inputs of the ANN, and allows for accurate training of the feedforward controller with data from the real system. Since the collected data can be immediately used for training of the controller, this method can easily be used for online adaptation.

The adaptation of the feedforward controller is demonstrated first in a biomechanical model of the elbow controlled by two muscles: a flexor and an extensor. Subsequently, the adaptation technique is applied to one subject with a high level upper extremity FES system. 
A simple fuzzy PD controller is used for feedback only to assist in the data collection, and to demonstrate the effect of different co-contraction levels.

\section{Methods}

The model

The model used to test the adaptation technique is a two-dimensional model in the horizontal plane (without gravity), with one degree of freedom: elbow flexion-extension, and two muscles: brachialis and lateral head of the triceps. The range of motion is from 0 to 170 degrees. The muscle and joint parameters were taken from cadaver studies performed by Klein-Breteler et al. (1999). The muscle model is a Hill-type model with two states: activation and muscle fiber length, and three elements: contractile, series elastic and parallel elastic, developed by McLean et al. (2003).

Some of the model parameters were modified in order to simulate possible differences between the model and the arm of the subject. The maximum muscle forces were reduced, and the muscle lengths were increased, as in the case of a weaker individual with longer limbs. These changes are shown in table 6-1.

\begin{tabular}{|l|l|l|}
\hline & Original model & Modified model \\
\hline Flexor maximum force & $1039 \mathrm{~N}$ & $208 \mathrm{~N}(20 \%$ of original $)$ \\
\hline Extensor maximum force & $1144 \mathrm{~N}$ & $343 \mathrm{~N}(30 \%$ of original $)$ \\
\hline Flexor muscle length at zero angle & $0.13774 \mathrm{~m}$ & $0.14774 \mathrm{~m}$ \\
\hline Extensor muscle length at zero angle & $0.21641 \mathrm{~m}$ & $0.22641 \mathrm{~m}$ \\
\hline Flexor optimal fiber length & $0.0738 \mathrm{~m}$ & $0.0938 \mathrm{~m}$ \\
\hline Extensor optimal fiber length & $0.0691 \mathrm{~m}$ & $0.0891 \mathrm{~m}$ \\
\hline
\end{tabular}

Table 6-1: Parameters of the original model modified to simulate a different individual. 
The model can run both inverse and forward dynamic simulations. In a forward dynamic simulation, the inputs are the muscle activations, and potential external forces, and the output is the resulting elbow angle; this type of simulation resembles the behavior of the human arm. In an inverse dynamic simulation, which can be used for control, the inputs are the desired angle and external forces, and the outputs are the required activations of the two muscles. In order to distribute the forces to the muscles, an optimization routine is performed that minimizes the muscle energy consumption according to Praagman et al. (2006).

\section{Controller}

The controller design is shown in figure 6-1. It consists of a feedforward part, which is an artificial neural network trained to approximate the inverse dynamics of the arm (or arm model), and a feedback part, which is a fuzzy PD controller that uses linguistic rules to correct the error between the goal and output elbow angle.

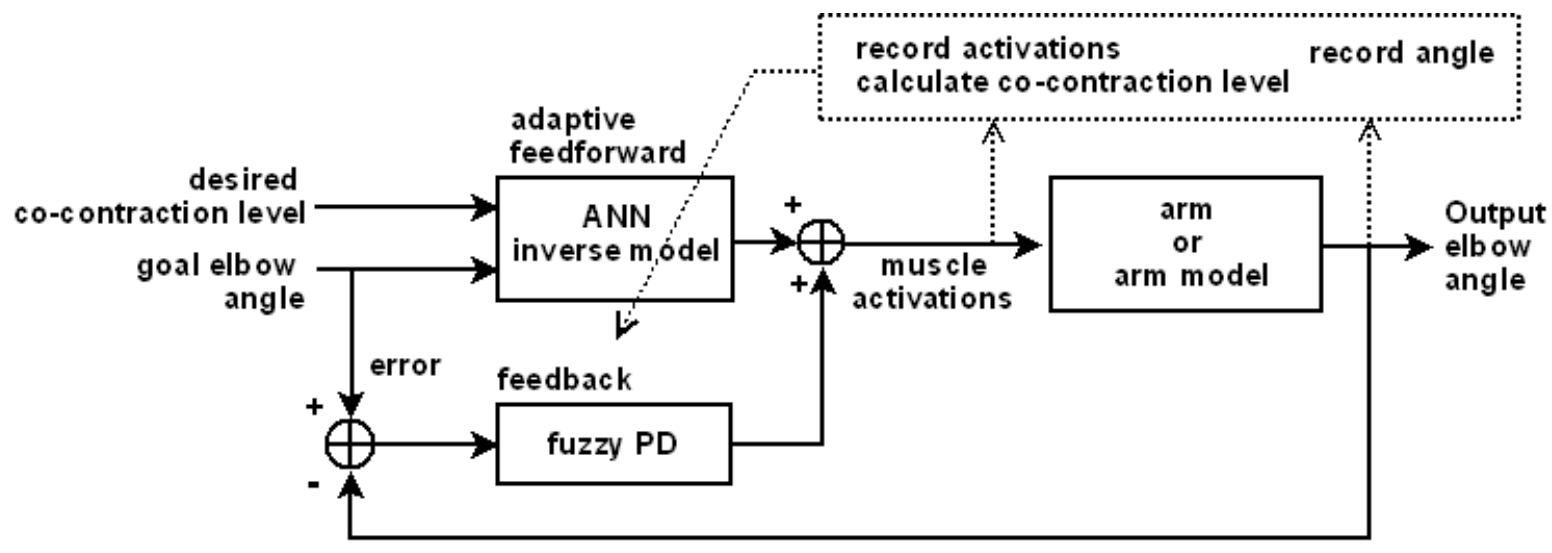

Figure 6-1: The controller design, including the adaptation scheme 
The ANN in the feedforward part of the controller is initially trained using the inverse dynamic model. The movements used for training are bell-shaped velocity movements that cover the range of the elbow angle. The training of the ANN is evaluated using the root mean square error between the muscle activations calculated by the model and the ANN:

$R M S E=\sqrt{\frac{\sum_{i=1}^{N}\left(y^{A N N}{ }_{i} y^{\bmod e l}{ }_{i}\right)^{2}}{N}}$

The fuzzy PD controller has three triangular input membership functions: positive, zero and negative, for the error and the error derivative, and five triangular output membership functions: large decrease, small decrease, no change, small increase and large increase, for the flexor and extensor activation change. These combine into nine rules of the form:

$I F$ error is positive $A N D$ error derivative is positive $T H E N$ flexor act. change is large increase

\section{Adaptation and co-contraction level}

After the model modifications listed in table 6-1, the ANN needs to be retrained according to the inverse dynamics of the new model. Assuming the modified model is the real human arm, an inverse dynamic model is not available, and therefore data from the forward dynamic model (i.e. the arm) have to be used for adapting the ANN. Since there are two muscles and one degree of freedom, the inverse is not unique, which means that the same elbow angle can be achieved with different pairs of muscle activations. In order to 
distinguish among all the correct activation pairs, another input is added to the ANN: the cocontraction level, a number between 0 and 1, unique for every pair of muscle activations.

The co-contraction level of muscle activations $a c t_{1}$ and $a c t_{2}$ is given by the following equation:

$$
\text { co-contraction }=\frac{a c t_{2}}{1-a c t_{1}+a c t_{2}}, a c t_{1} \geq a c t_{2}
$$

When one muscle has maximum activation, and the second has zero, the co-contraction level is set to zero (undefined according to the above equation).

The geometrical representation of the co-contraction level is shown in figure 6-2. The horizontal and vertical axes are the activation levels of the flexor and extensor muscles respectively. Since the muscle activation range is 0 to 1 , the only possible pairs of activations are inside the square with side length 1 . The iso-co-contraction lines go from $(0,1)$ to $(1,0)$, and are symmetrical with respect to the $y=x$ axis (dotted line). The $0,0.5,0.75$ and 1 iso-cocontraction lines are shown in the figure. (The 0 and 1 lines coincide with the edges of the square.) The dashed line a-d is an example of an iso-torque line, which means that any pairs of activations along that line would produce the same torque, according to the equation:

torque $=c_{1} \bullet$ force $_{1}+c_{2} \bullet$ force $_{2} \approx c_{1} \bullet a c t_{1}+c_{2} \bullet \bullet a c t_{2}$

where the relationship between activation and force is assumed to be approximately linear. Defining the co-contraction level makes the pair of activations unique: for example, the cocontraction level for point a is 0 , for $\mathrm{b}$ it is 0.5 , for $\mathrm{c}$ it is 0.75 , and for $\mathrm{d}$ it is 1 . Note that the slope of the iso-torque line can only be positive, since the two muscles are antagonists, and therefore it will always cross all the iso-co-contraction lines. 


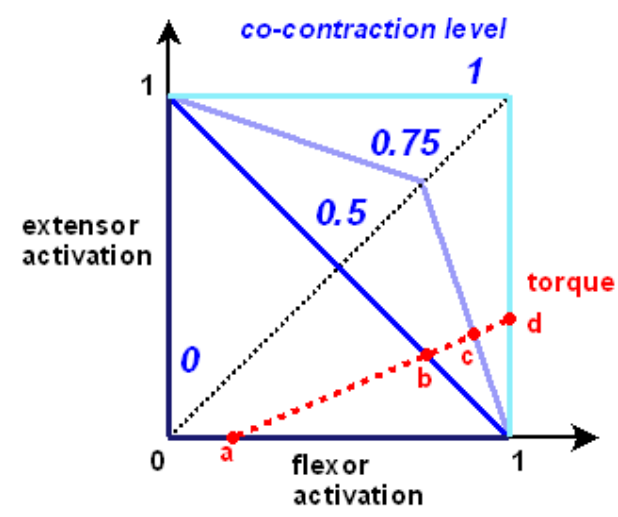

Figure 6-2: geometrical representation of the co-contraction level

The adaptation technique is shown in figure 6-1. Using the initial ANN as the controller, the input muscle activations and output elbow angle are recorded from the forward dynamic model. At this point, it is not important that the output trajectory follows the desired trajectory, as long as the resulting angle varies enough to provide adequate training data. If the initial ANN does not produce movement that covers the range of the elbow angle, the feedback controller is added to improve the trajectory tracking and result in better training data. From the recorded muscle activations, the co-contraction level is calculated, and the ANN is retrained using the elbow angle and co-contraction level as inputs, and the two muscle activations as outputs. The new ANN can then be used for control, more data can be collected, and the ANN can be adapted further, until the controller reaches an acceptable performance. In this study, the ANN was retrained until there was no significant difference between the performance of the ANN for the modified model, and the initial ANN for the original model. 
In order to demonstrate that the use of the co-contraction level as one of the inputs improves the training of the ANN, a second ANN was trained using the same data but without the co-contraction level as an input. The rest of the training parameters were exactly the same in both cases. After training was completed, the two ANNs were used for control with a set of goal trajectories not included in the training set, and their performance was compared based on the RMS error between the goal and output trajectory.

For the simulations mentioned above, the desired co-contraction level (for the ANN that used it as an input) was set to zero, because it causes the least amount of muscle fatigue. However, one set of trials using other co-contraction levels were performed, in order to examine the effect of co-contraction on the activation patterns and output angle. These trials used a fast bell-shaped velocity input, approximating a step, and the fuzzy PD feedback was added for more accurate tracking of the input. The co-contraction level was set to $0,0.5$, and 1. Moreover, to combine the advantages of different co-contraction levels, a simple variable co-contraction design was used with the same input. The desired co-contraction level linearly increased during the movement deceleration, and decreased when the deceleration went back to zero. This was not meant to be a test of the particular variable co-contraction design, but only an example of the potential benefits of using different co-contraction levels.

\section{Experimental evaluation}

The adaptation technique was evaluated in one subject who has received an implanted FES system following a hemisection lesion of the spinal cord (Brown-Séquard syndrome) at the highest cervical level. As a result of the injury, all motor function of the right upper extremity was lost, except for shoulder shrug, produced by the upper trapezius muscle. The 
FES system uses 24 channels to stimulate muscles of the shoulder, elbow and hand through muscle or nerve cuff electrodes. Muscle electrodes are placed on, or inserted into a muscle, while nerve cuff electrodes are placed around the nerve. The channels used in this study included one channel of the musculocutaneous nerve cuff electrode, that stimulates the brachialis muscle and causes elbow flexion, and one channel of the radial nerve cuff electrode, that stimulates the medial-lateral triceps and causes elbow extension. The subject gave informed consent prior to participation in the study.

The subject uses a mobile arm support to hold the arm up against gravity, because shoulder stiffness prevents the (stimulated) deltoid muscle from raising the arm. As a result, elbow flexion-extension takes place approximately in the horizontal plane, and is not affected by gravity. In order to measure the elbow flexion-extension angle, the Optotrak system (Northern Digital Inc.) was used. It consists of six cameras that record the position of light emitting diodes (LED) located inside the cameras measurement volume. A rigid body coordinate frame can be calculated if three or more LEDs are attached to the rigid body. For this study, two sets of eight LEDs were placed on the humerus and forearm, and the elbow flexion-extension angle was calculated from the relative position of the humerus and forearm coordinate frames.

The implanted stimulator delivers current with constant amplitude, and varying pulse width. In order to use the model-based controller described in the previous sections, the normalized activation levels that are the output of the controller need to be mapped to stimulation pulse width levels (in microseconds). The relationship between normalized activation and normalized stimulus is a sigmoidal function:

$$
a c t=\frac{1}{1+e^{-s t i m}}
$$


Normalized stimulus is then linearly mapped to pulse width (PW) using the minimum and maximum pulse widths:

$$
P W=\min _{P W}+\operatorname{stim} \bullet\left(\max _{P W}-\min _{P W}\right)
$$

The minimum pulse width is just below the stimulation threshold, which is the pulse width at which muscle contraction is first observed. The maximum pulse width is the highest value before spillover to other muscles or force plateau occurs.

The goal trajectories were similar to those used in simulation. They were bell-shaped velocity curves, with a range of 0 to 90 degrees, which was the flexion angle achieved with maximum brachialis stimulation. When the feedforward model-based controller was used in the subject, no movement was observed, due to the differences between the model and the subject arm, including but not limited to weakness of the paralyzed muscles, and arm stiffness. In order to collect data to retrain the feedforward controller, the fuzzy PD feedback loop was added. 50 trials lasting 25 seconds each were performed, with 35 seconds rest between trials to avoid fatigue. Subsequently, the data collected were used to train two ANNs, one that included the level of co-contraction as one of the inputs, and one that did not. Finally, the two ANNs were used to control the elbow angle in 50 trials (different than the ones used for training). The feedback loop was not included in these trials. The desired cocontraction level (for the ANN that used it as an input) was set to zero. Based on the RMS error calculated for each trial, the performance of the two ANNs was compared. 


\section{Results}

Figure 6-3 shows the effect of the model modifications on the feedforward controller performance, as well as an example of a training movement. The controller was trained on the original model (light colored solid line, in the top panel). When the same controller was used in the modified model, little movement was produced (black dashed line). So the feedback loop was added (light colored dashed line), to assist in moving the elbow through its range of motion, and collect useful data for the feedforward controller adaptation.
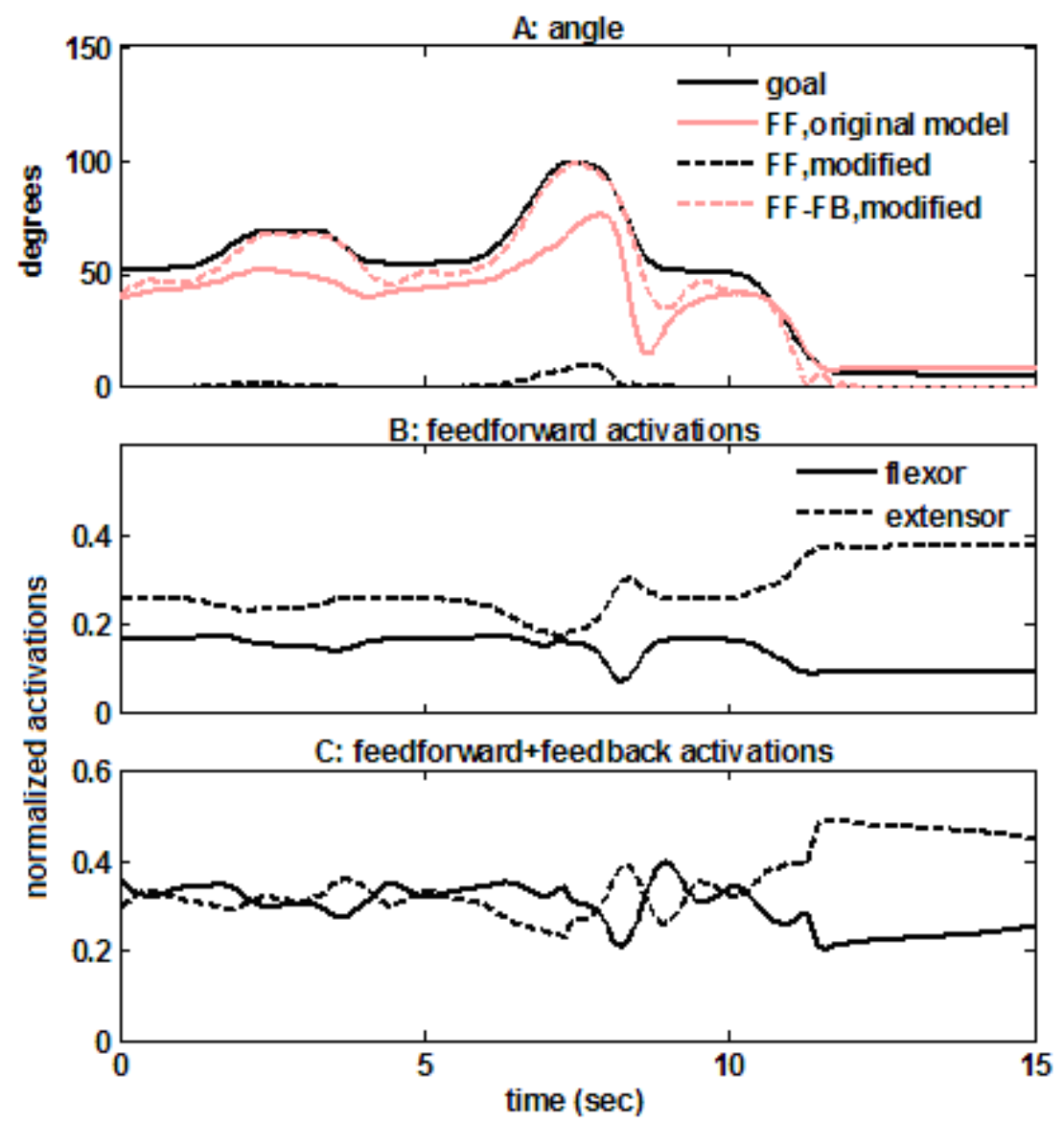

Figure 6-3: The original controller applied to the original and the modified model. Panel A shows the goal and output angles, the panel B shows the feedforward activations (the same in the original and modified model), and panel $\mathrm{C}$ shows the combined feedforward and feedback activations, used for the modified model. 
Figure 6-4 shows an example of the comparison between the two ANNs trained with and without the co-contraction level as an input. The RMS error for the ANN that includes and does not include the co-contraction level was 21.7 and 49.2 degrees, respectively.
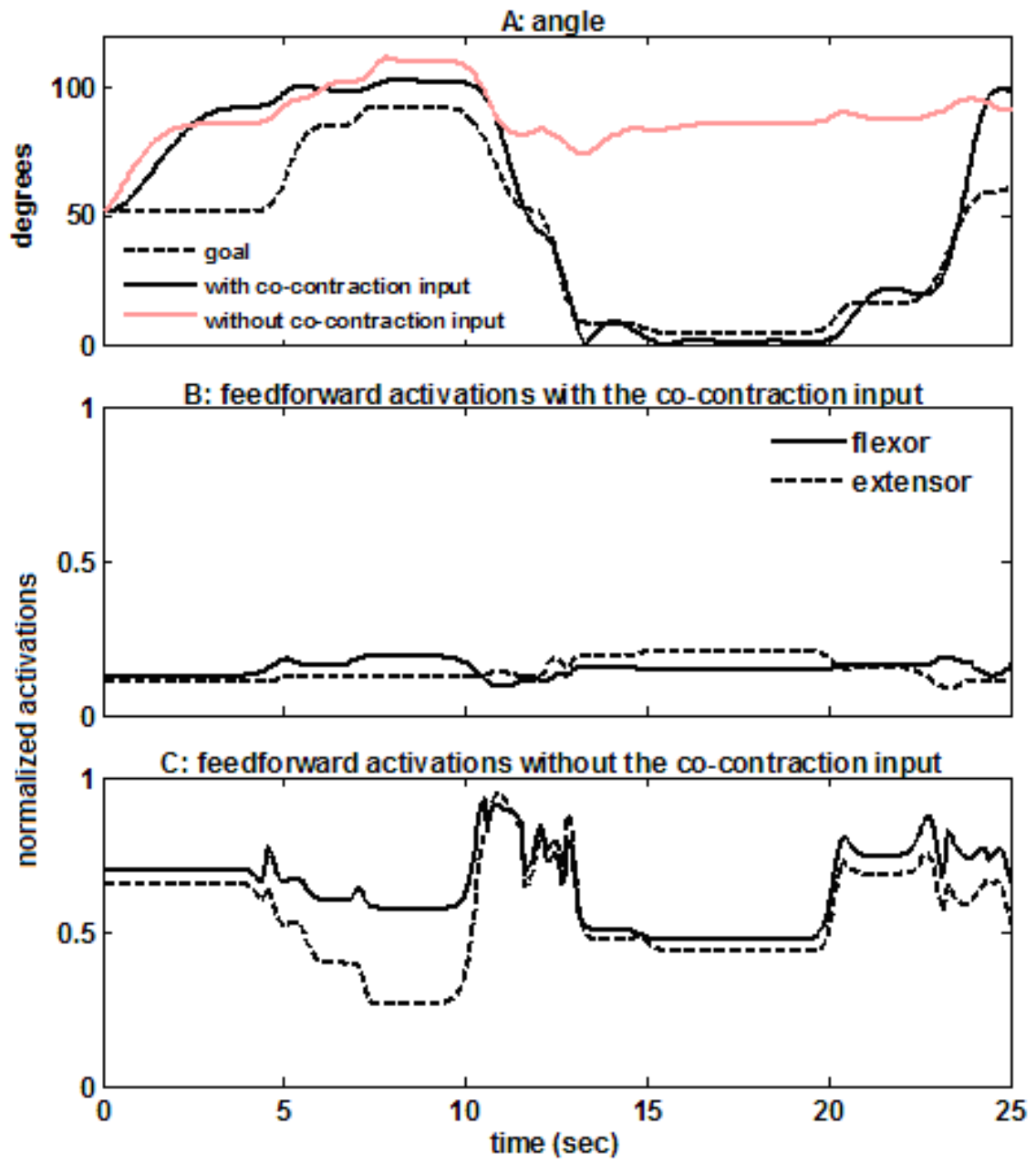

Figure 6-4: feedforward control including or not including the co-contraction level as an input. Panel A shows the goal and output angles, panel B shows the activations calculated by the controller with desired co-contraction level of 0 , and panel $\mathrm{C}$ shows the activations calculated by the controller that did not specify a co-contraction level. 
Figure 6-5 summarizes the performance of the various feedforward controllers. The bar labeled "modified model" is the mean RMS error of the original controller used in the modified model, without retraining. The next two bars are the mean errors of the controllers trained without and with the co-contraction level as an input. The bar labeled "original model" is the mean error of the original controller used on the original model. There is no significant difference between the "retrained with co-contraction input" and "original" controllers $(\mathrm{p}>0.1)$. There is a significant difference between all other pairs of controllers $(\mathrm{p}<0.05)$.

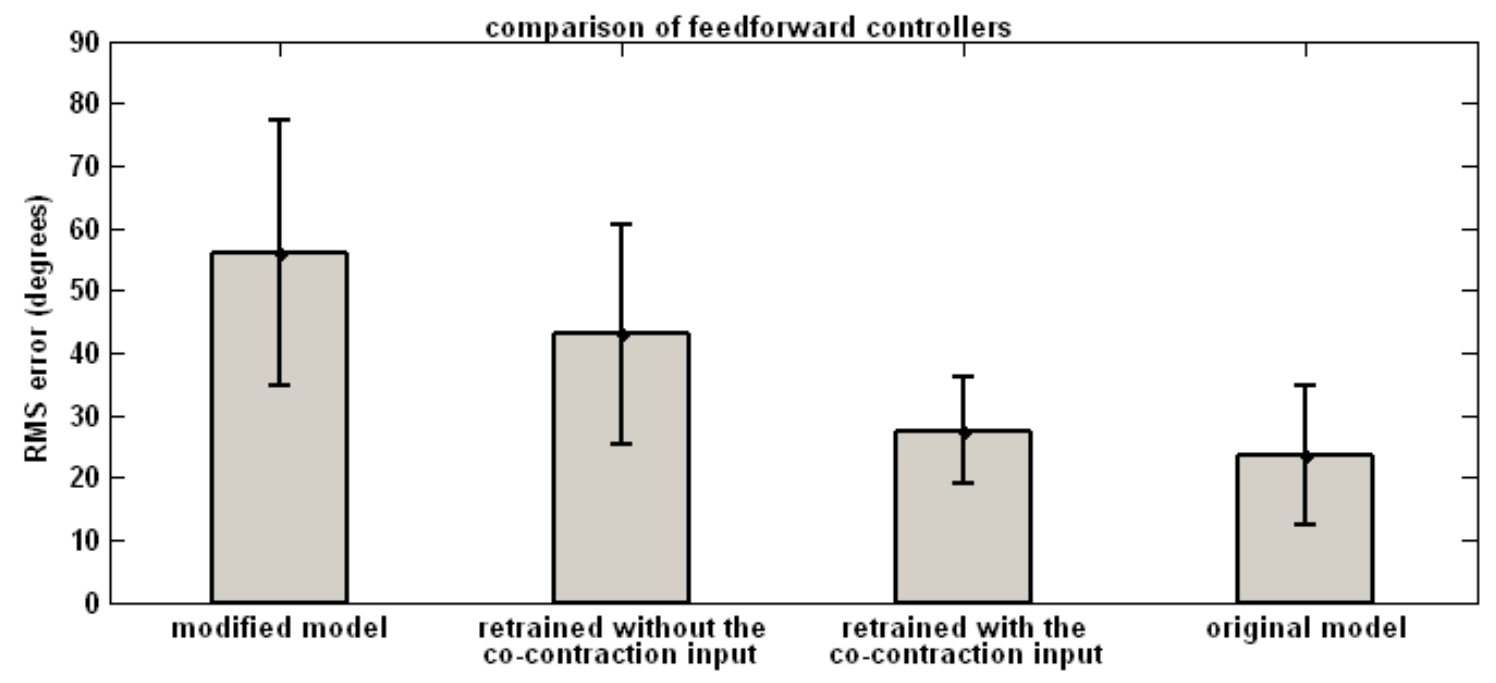

Figure 6-5: Comparison of the performance of different feedforward controllers. From left to right: the original controller used on the modified model, the controller trained without the co-contraction input on the modified model, the controller trained with the co-contraction input on the modified model, and the original controller on the original model (the ideal case).

Figure 6-6 shows the outcome of the trials that specified different levels of cocontraction for the same movement. The levels were $0,0.5,1$, and variable between 0 and 1 (shown in panel F). The fuzzy PD feedback was included in these trials. 

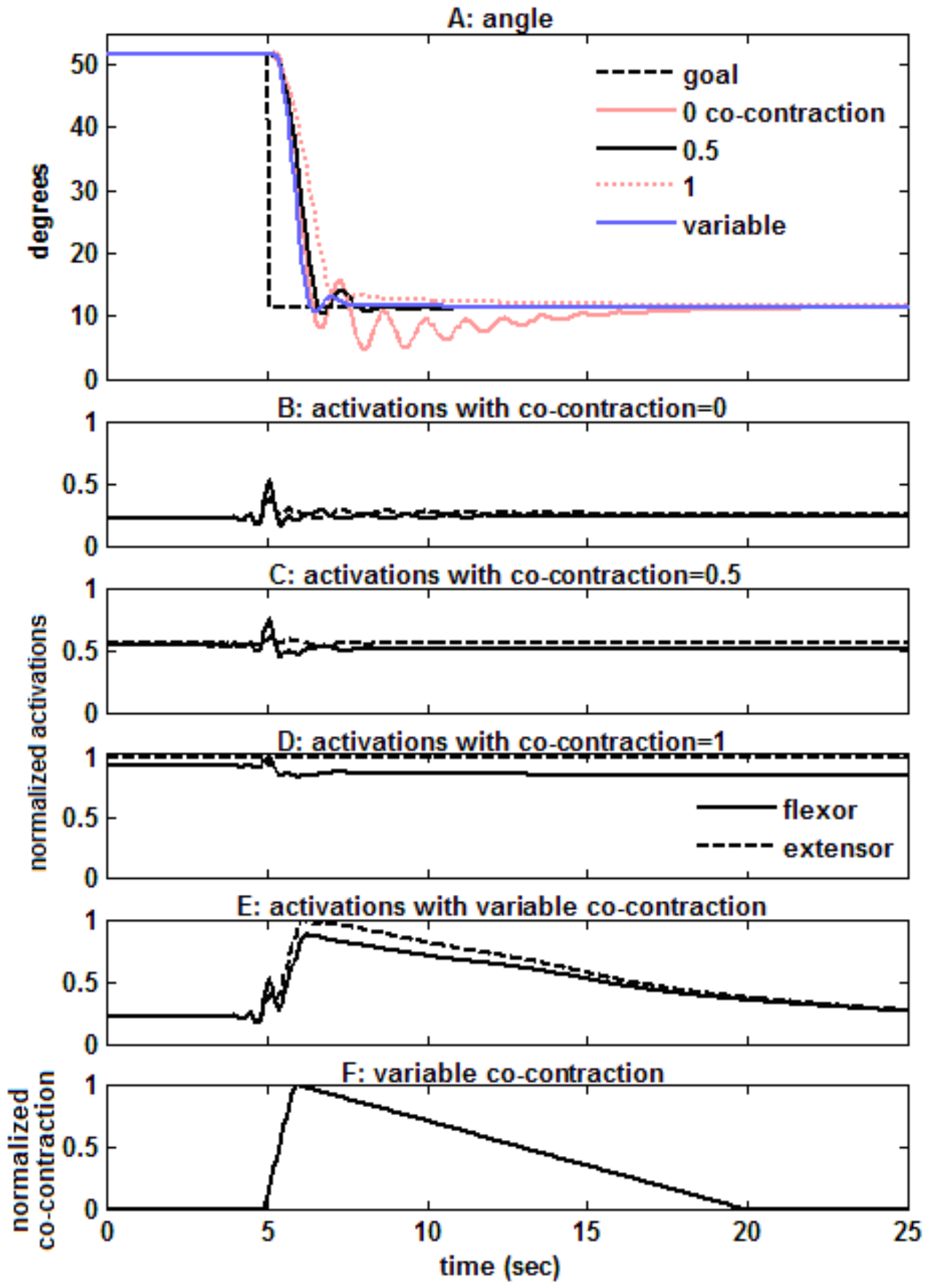

Figure 6-6: Effect of different co-contraction levels used by the feedforwardfeedback controller. Panel A shows the goal and the output angle with cocontraction of $0,0.5,1$ and variable. The corresponding muscle activations are shown in panels B-E. The variable co-contraction level is shown in panel F. 
Figure 6-7 shows an example of the data collected for training of the feedforward controller in the high level tetraplegia subject. Since the model-based ANN did not produce any arm movement, the fuzzy feedback loop, also model-based, was added to the controller. The tracking performance is not important, as long as the elbow is moved through its range.
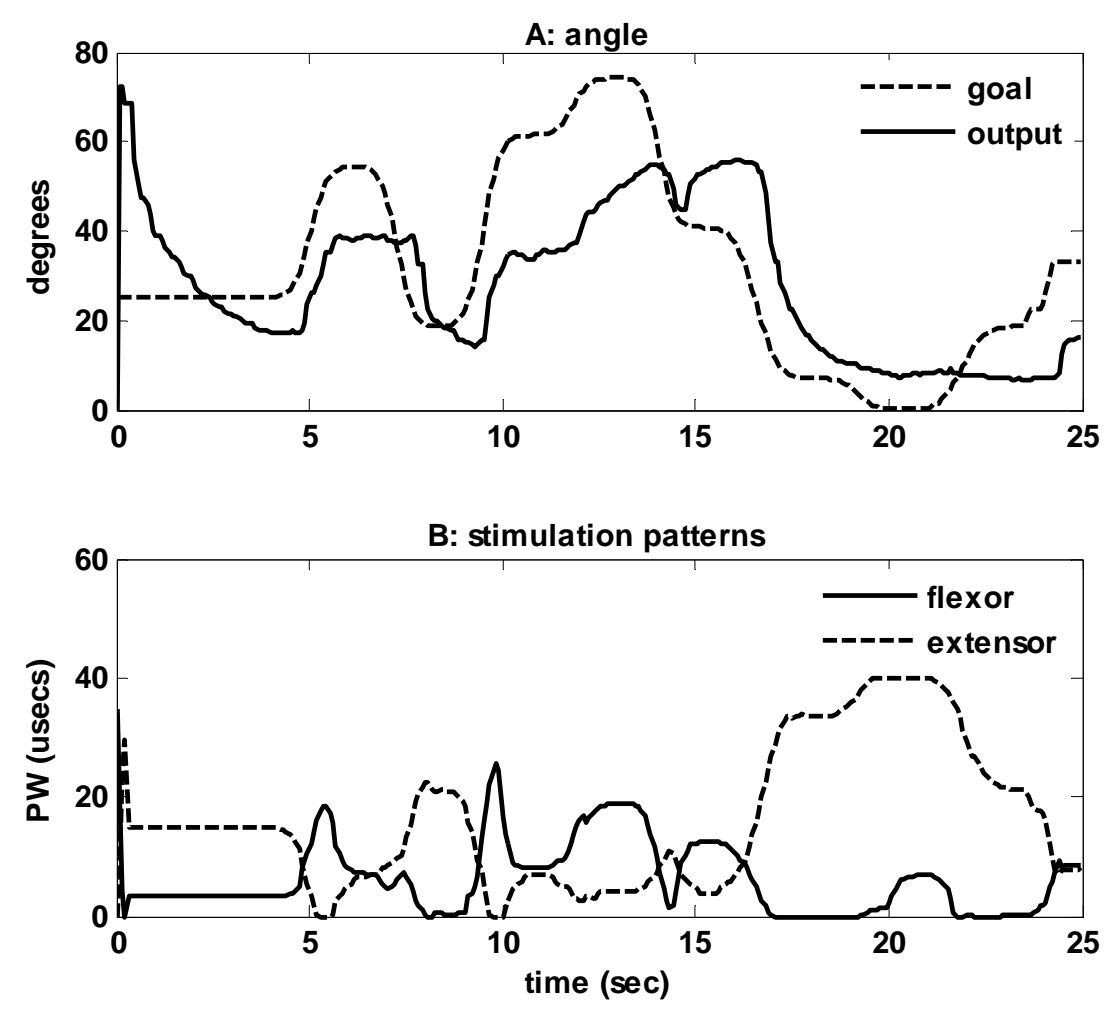

Figure 6-7: An example of data collection from the subject for the training of the feedforward controller, using the model-based feedforward-feedback controller. Panel A shows the goal and output elbow angle, and panel B shows the stimulation levels for the flexor (brachialis) and extensor (triceps). 
Figure 6-8 shows an example of a movement controlled using the ANN with specified co-contraction level of zero (black solid line, RMS error of 14.2 degrees), and the ANN that did not include the co-contraction level (light-colored solid line, RMS error of 20.0 degrees).
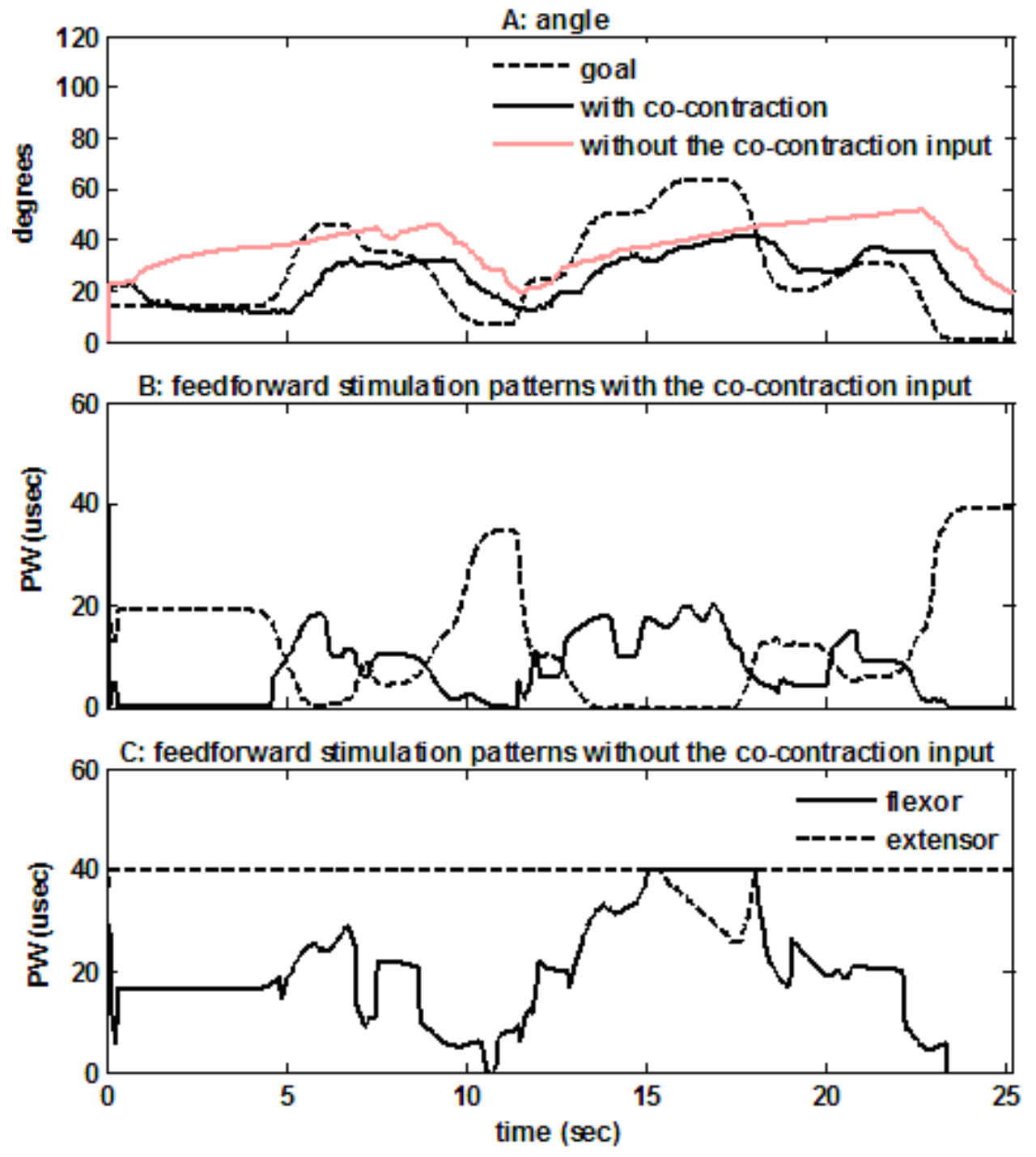

Figure 6-8: Example of an elbow movement performed by the subject using two different feedforward controllers: trained with and without the co-contraction level. Panel A shows the goal and output angles, panel B shows the muscle stimulation levels calculated by the controller with the co-contraction input, and panel $\mathrm{C}$ shows the stimulation levels calculated by the controller without the co-contraction input. 
Finally, figure 6-9 shows the mean RMS errors from fifty 25 -second trials performed using the two feedforward controllers. The errors are significantly different, $p<0.01$.

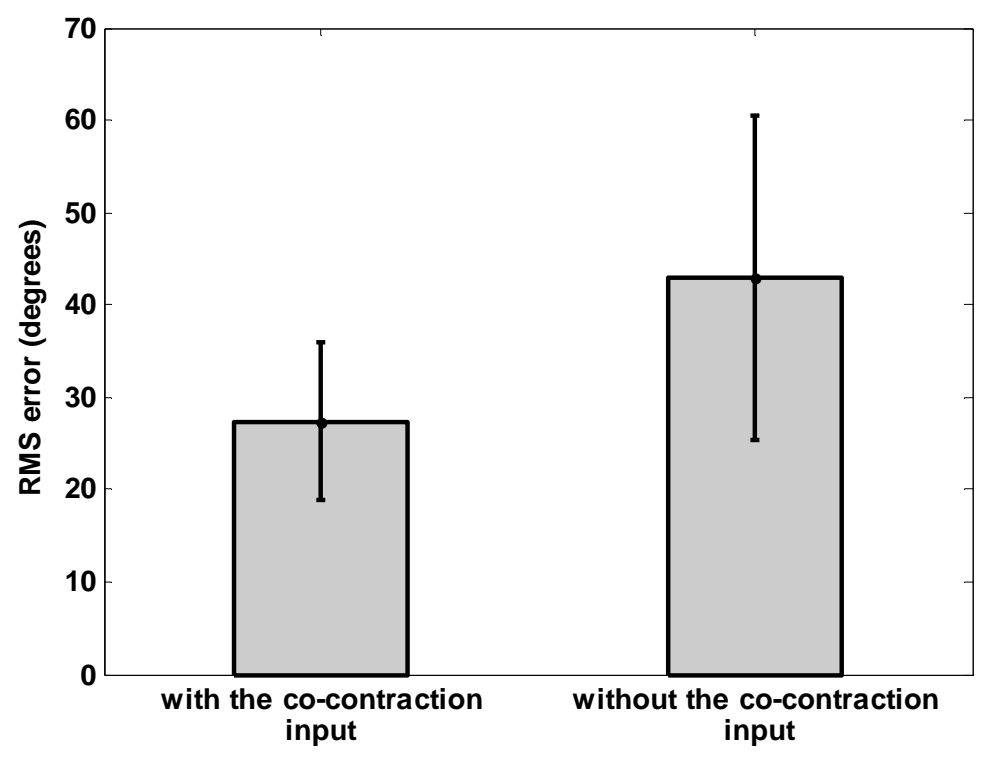

Figure 6-9: Mean error from fifty trials that used the feedforward controller with the co-contraction input (left) and without (right).

\section{Discussion}

The adaptation technique presented in this study improves the performance of the feedforward controller on a redundant system without a special training procedure, by simply using the data recorded while the system is in use. The more data are recorded, the more accurate the feedforward controller will be. This idea was first demonstrated in a model of the elbow joint, modified to simulate the differences that would be seen between a model and a real subject. Subsequently, when used in an actual FES system, the model-based controller that could initially produce no elbow movement had a final error of less than 30 degrees, comparable to the ideal model case.

The error of the feedforward controller, even in the ideal case, is too large to be an acceptable controller by itself. But as shown in figure 6-1, the feedforward is only one part of 
the controller, which also includes a feedback loop. Feedback corrects the activations in such a way as to minimize the trajectory tracking error; however by itself it can be too slow due to system delays (Stroeve 1996). So it is important to have a feedforward controller that is as accurate as possible. Moreover, in a more complicated system that would include the glenohumeral joint, the knowledge of the right activation patterns to ensure glenohumeral stability, learned by the feedforward controller by training on the inverse dynamics, could be crucial for the safety of the system.

As shown in figure 6-5, including the co-contraction level as one of the ANN inputs improved the learning of the ANN, since it created a unique inverse from the forward model data. This resulted in a lower tracking error compared to an ANN trained without the cocontraction level (mean of 26 and 45 degrees respectively). In addition, the activation patterns produced were consistent with the desired co-contraction level, as shown in panel B of figure 6-4, as well as panels B-D of figure 6-6. In contrast, the activations calculated by the ANN trained without co-contraction were much more variable with a tendency towards high co-contraction (panel C, figure 6-5).

Figure 6-6 shows a simple comparison of different co-contraction levels. For the zero co-contraction case, the response is under-damped, and increasing the co-contraction level increases the damping. The variable co-contraction was chosen so that it is low during the majority of the movement, and increases when the goal is reached. This is based on studies that have shown that co-contraction increases movement accuracy (Gribble et al. 2003), and in goal-oriented movements, accuracy is most important near the goal. Panel E shows that the variable co-contraction case combines the fast initial response of low co-contraction with the damping seen in the high co-contraction level. This indicates that using variable co- 
contraction of antagonistic muscles in FES control might benefit the movement accuracy of the system.

The experimental adaptation of the feedforward controller showed similar results to the simulations. The tracking error for the ANN with co-contraction was 27 degrees as opposed to 35 degrees without co-contraction. The difference in the activation patterns produced by the two ANNs is shown in figure 6-8. The maximum pulse width for both the brachialis and the triceps was $40 \mu \mathrm{sec}$, and the activations produced by the low cocontraction ANN were less than $50 \%$ of the maximum for most of the trial (panel B). In the case of the ANN without co-contraction, one or the other of the stimulation channels was at the maximum value for the entire trial (panel C), which is undesirable as it would quickly cause fatigue.

The adaptation technique is implemented in this study in a single pair of antagonistic muscles. In a larger system, different co-contraction levels might be used for every degree of freedom, for example, the same idea could be applied to the control of pronation-supination. For muscles that cross more than one degree of freedom, and cannot easily be divided into antagonistic pairs (e.g. muscles crossing the glenohumeral joint), a more complicated adaptation technique might be needed, that would combine co-contraction of antagonists with activation patterns among synergistic muscles. The goal would still be the same: to balance the number of muscles and degrees of freedom in order to make the inverse unique and allow adaptation of the feedforward controller with data from the actual system. In this way, the FES controller can be customized to the specific user and offer the best possible performance. The co-contraction method presented in this study is a positive step in that direction. 


\title{
Chapter 7: Performance of a feedforward-feedback FES controller in an individual with high tetraplegia
}

\begin{abstract}
A functional electrical stimulation (FES) system has been developed for restoration of upper limb function following a high cervical level spinal cord injury. Control of arm movement using appropriate stimulation patterns for paralyzed shoulder and arm muscles is a difficult task, due to the wide variety of potential restored motions, the shoulder stability issues, and the complicated action of muscles that cross multiple joints. In this study, a combined feedforward-feedback design is presented for shoulder and arm control of the FES system. The feedforward controller is a set of artificial neural networks that are trained with data collected during system use, to approximate the inverse dynamics of the arm. The feedback is a fuzzy PD controller which adjusts the stimulation levels based on the trajectory error and the muscle moment arms. The controller uses model-based activation patterns to calculate the stimulation levels for the scapular and glenohumeral stability muscles. The design is tested in one individual with an implanted FES system after a high cervical level spinal cord injury. Nine stimulation channels are used for control of shoulder movement, and elbow flexion-extension. The controller shows good tracking performance, with an endpoint error of less than $10 \mathrm{~cm}$ in position, and 25 degrees in orientation.
\end{abstract}

\section{Introduction}

Functional electrical stimulation (FES) restores function to individuals with spinal cord injury by electrically stimulating paralyzed muscles. FES applications have been 
developed to address various problems, in different injury levels. In paraplegia, FES has been used to assist in standing (Davis et al. 2001), walking (Marsolais and Kobetic 1987), and bladder function (Creasy et al. 2001). For individuals with mid-level tetraplegia (C5-C6), the main focus has been hand grasp and release (Handa and Hoshimiya 1987; Peckham et al. 1988; Popovic et al. 1999), but systems have also been designed to help with elbow flexionextension (Crago et al. 1998) and forearm pronation-supination (Lemay et al. 1996).

Few FES systems have addressed the problem of restoring shoulder and arm function in high cervical level tetraplegia (C1-C4). Hoshimiya et al. described a percutaneous system for shoulder, elbow, wrist and finger function in tetraplegia, which used pre-programmed stimulation patterns to restore a limited number of movements (Hoshimiya et al. 1989; Kameyama et al. 1999). Specifically for the shoulder, the system gave the choice of three stimulation patterns, based on EMG measurements of shoulder muscles in able-bodied individuals (Kameyama et al. 1993). This approach resulted in a very limited repertoire of motions available to the FES user, and in addition, it assumed that all the muscles used by an able-bodied person would be available for stimulation in an individual with high tetraplegia, which is usually not the case due to denervation.

Another issue with this system, and most FES systems in clinical use to date, is that they are purely feedforward: they use pre-defined stimulation patterns that cannot be adjusted to correct for errors in the system performance due to external disturbances or muscle fatigue. In single degree of freedom systems (e.g. hand grasp), this still allows for successful function, since the user can monitor the system output and adjust the command for better performance. However, in the case of a shoulder and arm neuroprosthesis, there are many degrees of freedom to be controlled, and complicated interactions between multi-joint 
muscles, so that the cognitive burden on the FES recipient could make the system prohibitively difficult to use.

This study presents a different approach to the control of shoulder and arm muscles in a high tetraplegia neuroprosthesis. Our design uses artificial neural networks (ANN) to implement direct inverse control, which allows for a wide range of arm movements instead of pre-programmed stimulation patterns. Moreover, all that is required of the user is to give the command for the desired trajectory; the controller includes a fuzzy PD feedback loop that monitors the resulting movement, and automatically adjusts the stimulation levels to correct for errors. Finally, it can record data while the system is in use, and later use them to adjust the ANN parameters so that the controller is customized to the muscle strength and characteristics of the specific individual. The system is implemented in one subject with an implanted high level upper extremity neuroprosthesis.

\section{Methods}

The subject

The subject has an FES system for restoration of motor function on the right upper limb, which was paralyzed following a hemisection lesion of the spinal cord (Brown-Séquard syndrome) at the highest cervical level. The subject gave informed consent prior to participation in the study.

The FES system includes 24 channels for stimulation of shoulder, arm and hand muscles. Nine of these channels are used in this study and are listed in table 7-1. Most of the channels are nerve cuff electrodes, which wrap around the nerve and stimulate muscles innervated by that nerve. Three are intramuscular electrodes, which are inserted into a 
specific muscle. Of the nine electrodes, seven are used for shoulder control, and two for elbow flexion-extension (the triceps and brachialis).

\begin{tabular}{|l|l|l|}
\hline Electrode type & Nerve & Muscle \\
\hline Nerve cuff & Axillary & Deltoid \\
\hline Nerve cuff & Long Thoracic & Serratus anterior \\
\hline Nerve cuff & Thoracodorsal & Latissimus dorsi \\
\hline Nerve cuff & Suprascapular & Infraspinatus-Supraspinatus (rotator cuff) \\
\hline Intramuscular & Rhomboid \\
\hline Intramuscular & Upper pectoralis major \\
\hline Intramuscular & & Lower pectoralis major \\
\hline Nerve cuff & Radial & Medial-lateral triceps \\
\hline Nerve cuff & Musculocutaneous & Brachialis \\
\hline
\end{tabular}

Table 7-1: The stimulation channels

Four angles are used for feedback control: humerus plane of elevation, elevation angle, rotation angle, and elbow-flexion extension. Forearm pronation-supination is not measured because the subject uses a mobile arm support to hold the arm against gravity, due to considerable shoulder stiffness, and this arm support does not allow rotation of the forearm. The angles are calculated based on an Optotrak motion tracking system (Northern Digital Inc.). This system consists of six cameras that record the position of light emitting diodes (LED) located inside the cameras' measurement volume. Sets of three or more LEDs attached to a rigid body can be used to calculate a coordinate frame. For this study, three sets of eight LEDs were placed on the thorax, humerus and forearm. The relative orientation of the thorax and humerus coordinate frames was used to calculate the three shoulder angles, and the relative orientation of the humerus and forearm was used to calculate the elbow flexion-extension angle. The movements of the clavicle and scapula were not recorded, because they are difficult to measure in real time using a marker-based system like the 
Optotrak in the lab, or any set of sensors that would be part of a portable take-home system. It is the responsibility of the FES controller to appropriately stimulate the clavicular and scapular muscles in order to ensure scapular stability and create a natural scapulo-humeral rhythm.

The implanted stimulator delivers current with constant amplitude, and varying pulse width. The model-based muscle activation patterns (that range from 0 to 1) are mapped to pulse width levels through a sigmoidal function:

$$
a c t=\frac{1}{1+e^{-s t i m}}
$$

scaled by the minimum and maximum pulse widths:

$$
P W=\min _{P W}+\operatorname{stim} \bullet\left(\max _{P W}-\min _{P W}\right)
$$

The minimum pulse width is just below the stimulation threshold, which is the pulse width at which muscle contraction is first observed. The maximum pulse width is the highest value before spillover to other muscles, force plateau, or a feeling of discomfort occurs. Discomfort is usually not an issue with paralyzed FES users, but the unusual nature of this subject's injury resulted in motor paralysis but normal to hypersensitive sensory function.

\section{The controller}

The controller design is shown in figure 7-1. The feedforward controller is two artificial neural networks (ANNs), one for the shoulder and one for the elbow, trained according to the inverse dynamics of the arm. The initial ANNs are model-based, but adaptation is needed in order to account for the differences between the arm model and the actual arm of the subject. This is achieved using data recorded from the arm, during normal 
function of the FES system. Since there are more muscles (seven and two, for the shoulder and elbow) than degrees of freedom (three and one), it is not possible to simply invert the arm data to train the inverse-model-ANNs, because the inverse is not unique.

In the case of the elbow ANN, the problem is solved by adding the desired cocontraction level of the triceps and brachialis, as one of the inputs. The inverse network then has two inputs (elbow angle and co-contraction level) and two outputs (brachialis and triceps activation), and can be successfully trained with data from the (forward) actual arm function. The co-contraction level is defined as:

co-contraction $=\frac{a c t_{2}}{1-a c t_{1}+a c t_{2}}, a c t_{1} \geq a c t_{2}$

and zero when one muscle is activated maximally, and the other zero (undefined according to the above equation).

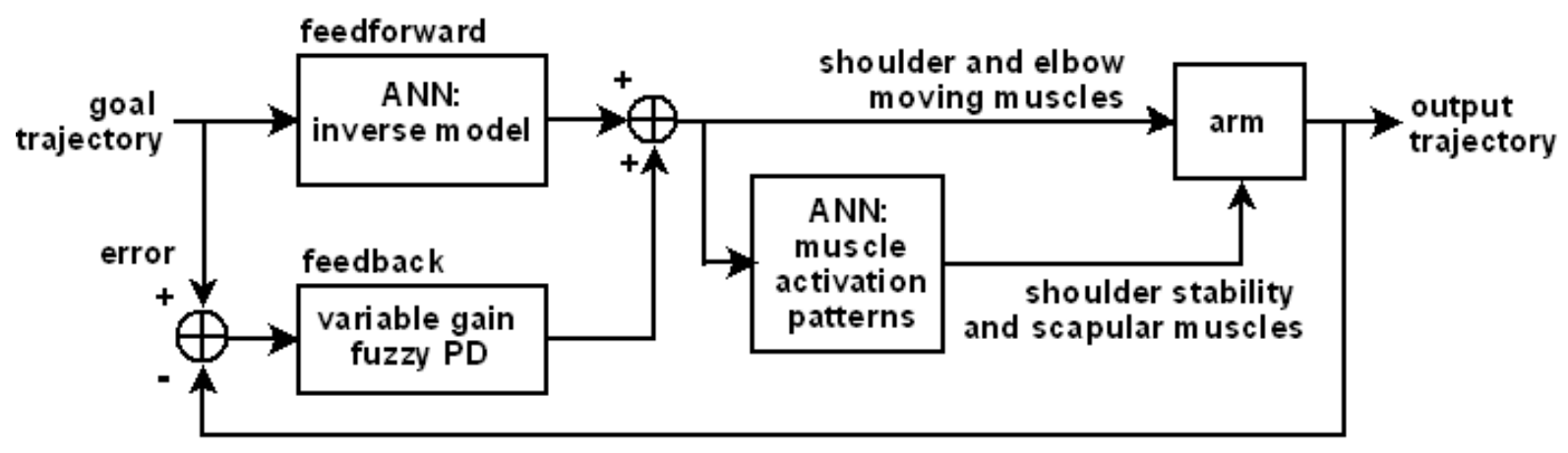

Figure 7-1: The controller design

In the case of the shoulder ANN, a similar input can be added for the deltoidlatissimus dorsi antagonist pair. However, the ANN still has fewer inputs (three degrees of freedom and co-contraction level) than outputs (seven muscle activations). To solve this problem, the shoulder muscles are divided into two categories. The first is the prime shoulder 
movers, whose activation levels directly affect the shoulder angles, and this includes the deltoid, latissimus dorsi, and upper and lower pectoralis major. The second category includes the scapular muscles (serratus anterior and rhomboid) and the glenohumeral stability muscles, i.e. the rotator cuff. The muscles of the second category have more of a stabilizing role on the scapula and humerus, and do not cause as much movement of the shoulder. However, their activation is closely related to that of the shoulder moving muscles. For the scapular muscles, this relationship originates from the scapulo-humeral rhythm: during a normal arm elevation movement, about one-third is due to scapulothoracic motion, and twothirds is due to glenohumeral rotation (de Groot and Brand 2001). Activation of the glenohumeral stability muscles aims to protect the glenohumeral joint from the de-stabilizing effect of the shoulder moving muscles (and potential external forces). In both cases, activation of the muscles in the second category is dependent on the activation of the muscles in the first. Based on this fact, only the muscles of the first category are used as outputs of the adaptive feedforward ANN.

The activations of the stabilizing muscles are calculated from the activations of the shoulder moving muscles. The patterns of activation were taken from a modeling study. First, a musculoskeletal model of the upper extremity was customized to the subject, i.e. the only muscles allowed to produce force were the ones stimulated by the FES system and the maximum force produced by those muscles was reduced from the able-bodied case. Then, a large number of inverse dynamic simulations were performed, using as inputs a wide variety of reaching movements in the arm workspace. The outputs were the activation patterns of all the stimulated shoulder muscles, which were then divided into the prime movers and the 
stability muscles. These sets of activation patterns were used to train an ANN, which learned to predict the stability muscle activations from the shoulder moving muscle activations.

Adaptation of the elbow feedforward controller was presented in detail in the previous chapter. In order to record data to train the shoulder feedforward controller, various patterns of stimulation of the four shoulder moving muscles were applied, which, in turn, resulted in stimulation of the stabilizing muscles, according to the relationship described in the previous paragraph. These patterns of stimulation included bell-shaped curves with a maximum chosen randomly between zero and the maximum pulse width recorded for that muscle, either for a single muscle, or a combination of muscles. The stimulation patterns and the resulting shoulder angles were recorded and used for ANN training.

As seen in figure 7-1, the controller also includes a feedback loop, which is a variable-gain fuzzy PD controller. The error and the error derivative have three triangular membership functions (positive, zero and negative), which combine into nine fuzzy rule antecedents. The rule consequents for each stimulation channel depend on the model-based moment arm of the corresponding muscle at the current position. For example, if a muscle moment arm is positive for a degree of freedom in a specific position, positive error will result in an increase of that muscle activation; if the moment arm is negative in a different position, it will result in a decrease. The fuzzy controller was preferred to traditional PD control, because we were not able to find appropriate values for the traditional PD gains during an experimental session, typically limited to 3-4 hours. The performance of the fuzzy controller appeared to be less sensitive to the choice of membership functions, and was superior to the performance of the traditional PD controller under the experimental time constraints. 


\section{Controller evaluation}

The controller for the elbow only was used initially to test the feedforward-feedback design. Goal-oriented movements (with bell-shaped velocity profiles), steps and sinusoids were used as input trajectories, and the controller performance was quantified using the root mean square error between the goal and output movement:

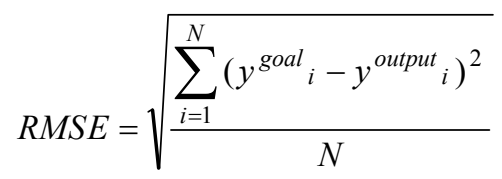

The elbow controller was also used to determine the most appropriate movement velocity, using as inputs sinusoids of different frequencies and choosing the best compromise between speed and trajectory tracking error.

Subsequently, the feedforward-feedback design was applied to the entire arm, with goal-oriented input movements inside the range of the arm angles. To calculate this range, maximum stimulation was applied to the muscles and the resulting angles were recorded. The controller performance was described by the endpoint position error: the distance between the desired and actual endpoint, and the endpoint orientation error: the angle between the desired and actual endpoint orientation.

In one of the testing sessions, we explored the variability caused by the experimental setup, as well as the arm itself. A predefined pattern of stimulation was applied to the muscles, and the resulting shoulder and elbow angles were recorded. Then the Optotrak markers were removed and put back on, and the stimulation pattern was applied again. Next, the arm was taken off the mobile arm support, and put back on, and the same stimulation pattern was delivered to the muscles. Finally, in the end of that testing session (about two 
hours later), during which the arm support and the Optotrak markers were not moved, the stimulation pattern was applied again. The RMS error was used to quantify the differences between the output angles due to the Optotrak markers, the mobile arm support, and the changes in the arm properties.

\section{Results}

Figure 7-2 shows an example of a sinusoidal movement for the elbow angle, controlled by three controllers: the feedforward only (light colored dashed line), the feedback only (black solid line) and the combination of feedforward and feedback (light colored solid line). The RMS errors for the three controllers were 13.9, 10.5 and 9.3 degrees, respectively. The activations for the brachialis (flexor) and triceps (extensor) calculated by the three controllers are shown in panels B, C and D. 

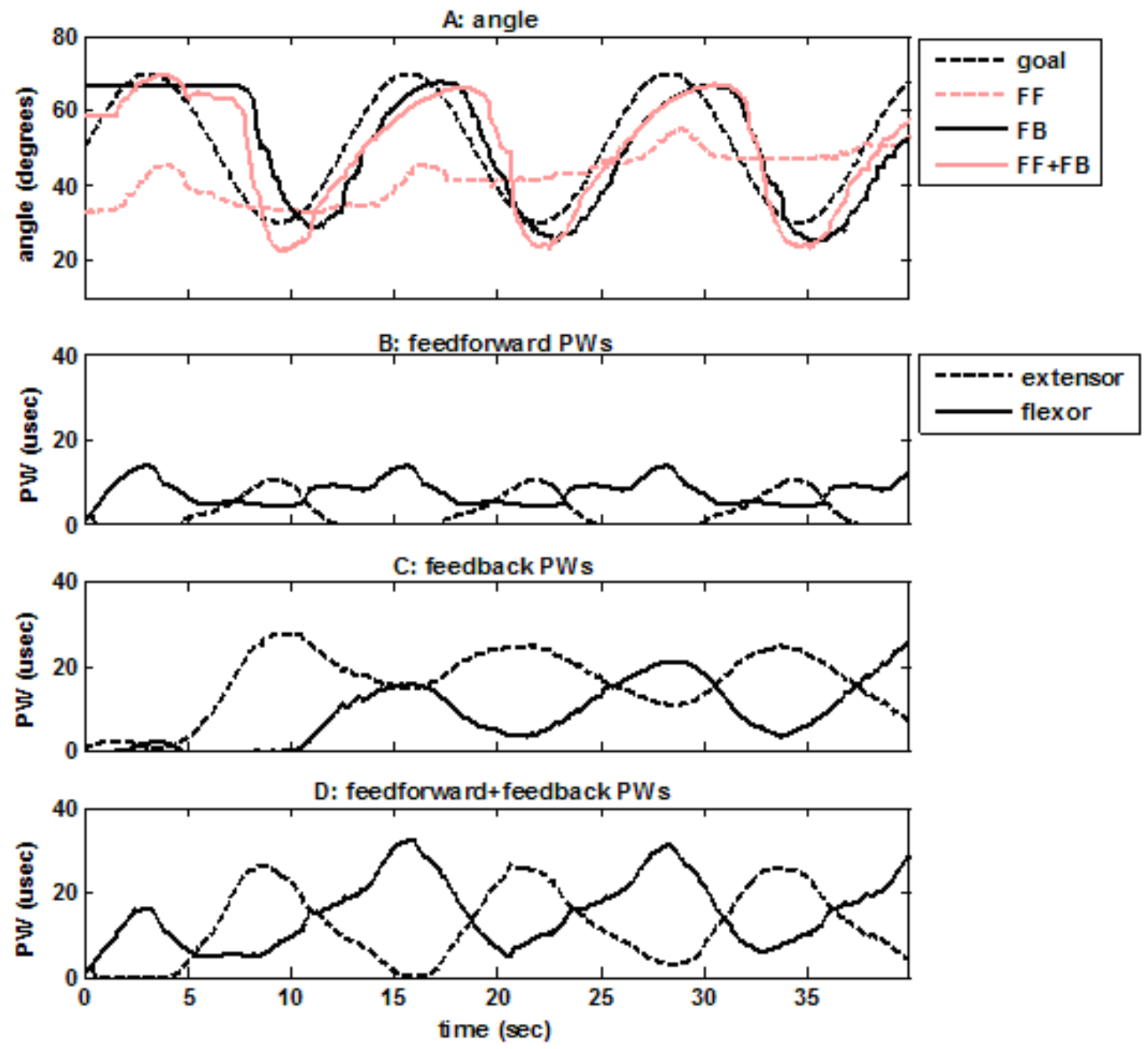

Figure 7-2: Example of a sinusoidal movement performed using three controllers. Panel A shows the goal and output angles, and panels B, C and D show the muscle stimulation levels calculated by the feedforward only, feedback only, and combination of feedforward and feedback controller, respectively. 
The error for the three controllers based on 30 trials is shown in figure 7-3.

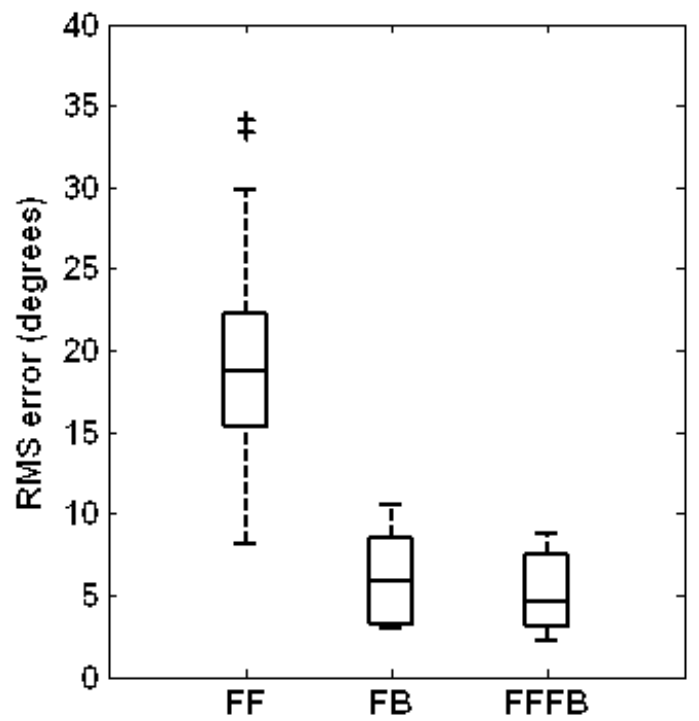

Figure 7-3: Elbow flexion-extension controller performance for the feedforward (left), feedback (middle) and feedforward-feedback controller (right).

The effect of different movement velocities on the controller performance is shown in figure 7-4. The input in the left column is a sinusoid of frequency $0.1 \mathrm{~Hz}$, and the resulting trajectory has an error of 3.3 degrees. The sinusoid on the right column has a frequency of $1 \mathrm{~Hz}$, and in that case the error is 14.4 degrees. 

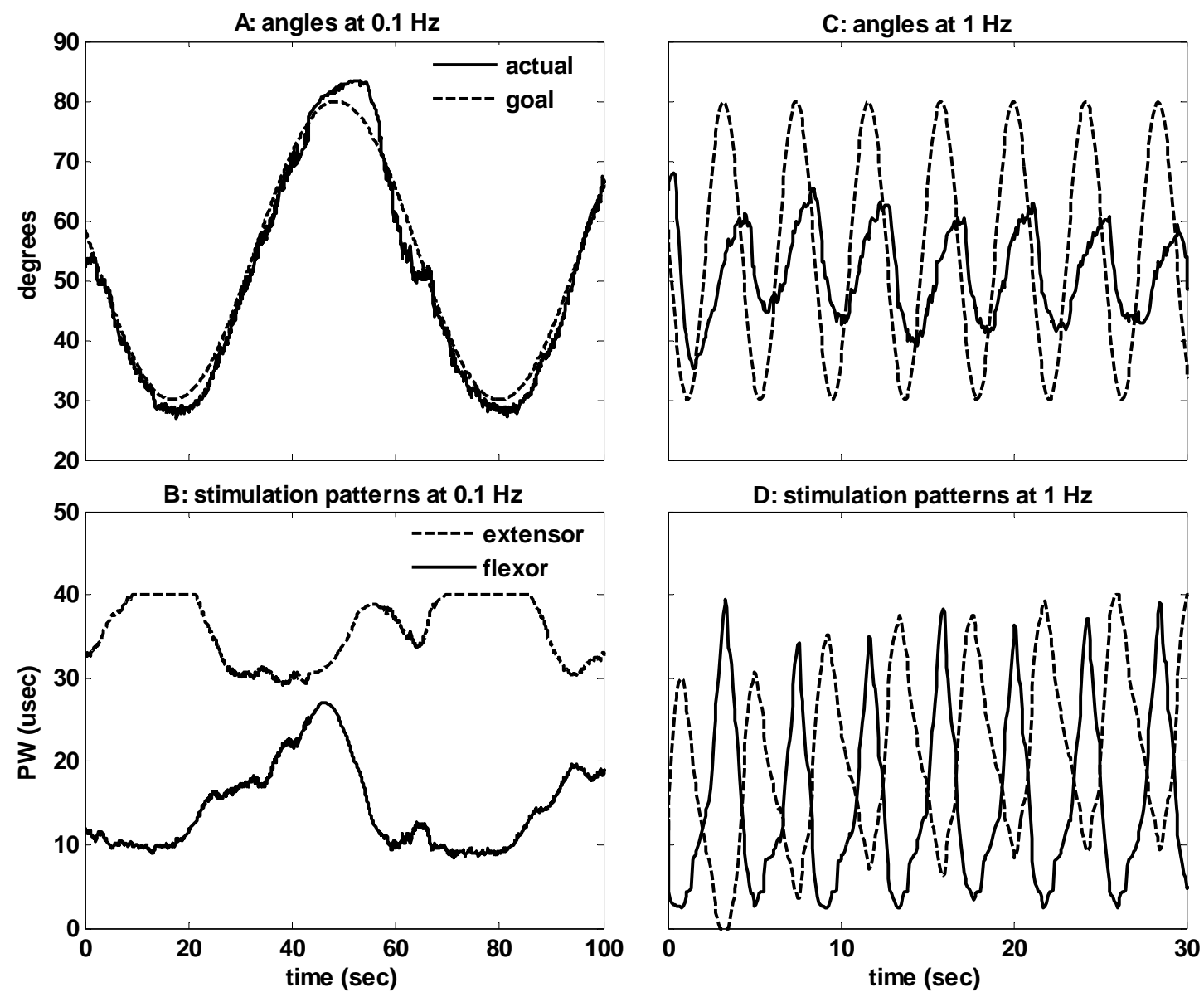

Figure 7-4: Example of sinusoidal inputs of different frequencies and their effect on the performance of the elbow controller. Panel A shows the goal and output angle at $0.1 \mathrm{~Hz}$, and panel $\mathrm{C}$ shows the angles at $1 \mathrm{~Hz}$. Panels B and D show the muscle stimulation patterns at $0.1 \mathrm{~Hz}$ and $1 \mathrm{~Hz}$ respectively.

The RMS errors for four different movement frequencies are shown in figure 7-5. Based on these errors, the preferred frequency for the movement inputs used in this study was around $0.5 \mathrm{~Hz}$.

Figure 7-6 shows an example of varying step inputs, used to calculate the error at the "goal" of a movement. Each step lasted for 5 seconds, and the error was calculated right before a new step was applied. 


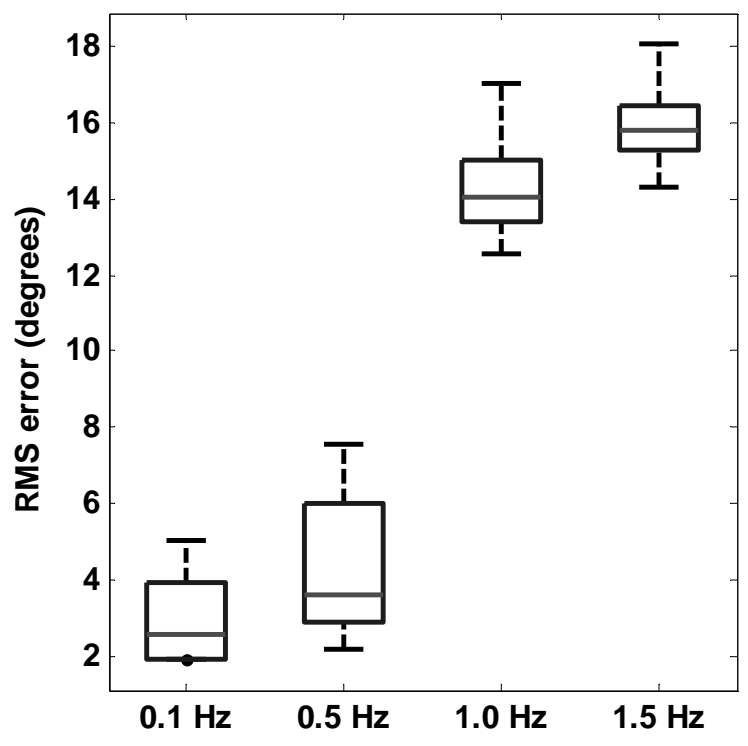

Figure 7-5: The errors for movements of four different frequencies.
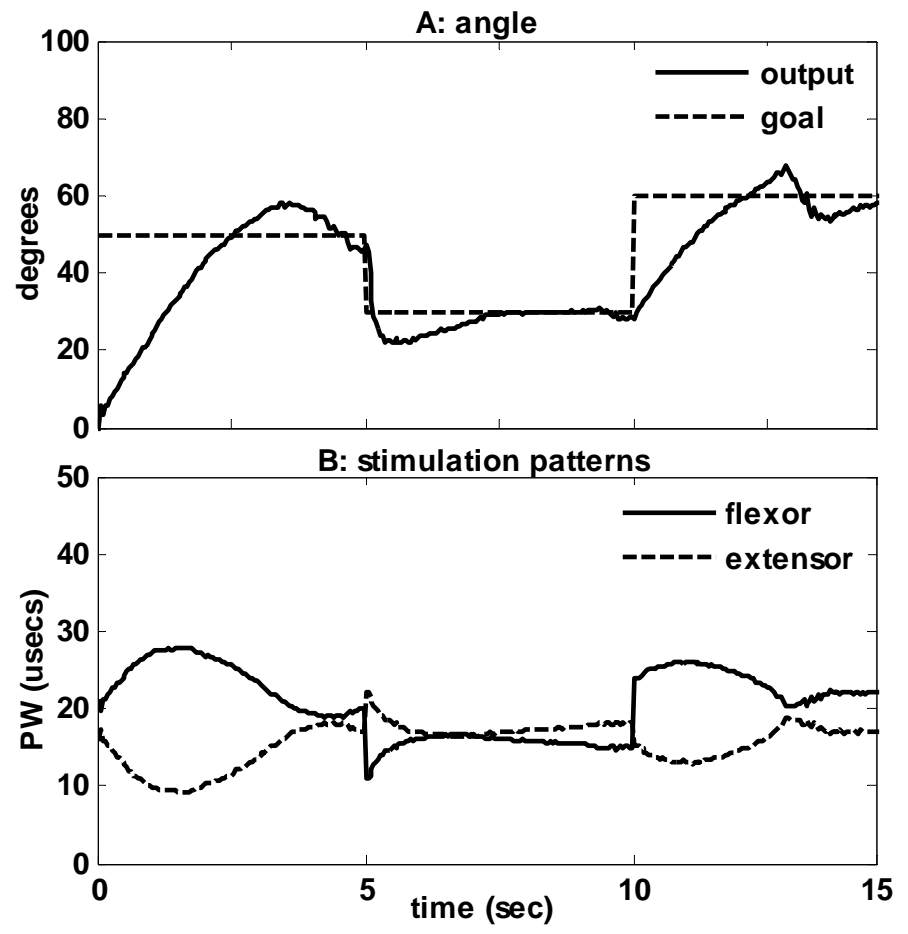

Figure 7-6: Example of elbow movements with step inputs. Panel A shows the goal and output angle, and panel B shows the stimulation levels for the elbow flexor (brachialis) and extensor (triceps). 
The error based on 30 trials of step inputs is shown in figure 7-7.

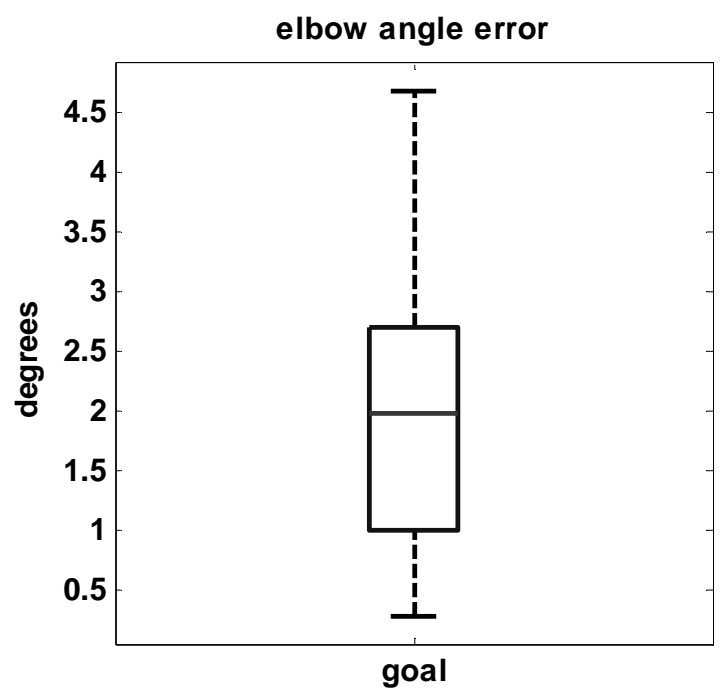

Figure 7-7: The error at the "goal" of the movement, calculated five seconds after a step input was applied.

An example of a goal-oriented movement for the entire arm is shown in figure 7-8. The shoulder goal and output angles are shown in panel A, and the goal and output elbow flexion-extension is shown in panel B. The endpoint position and orientation errors are shown in $\mathrm{C}$ and $\mathrm{D}$ respectively, and the stimulation pulse widths, normalized to the maximum of each one are shown in panel E. 

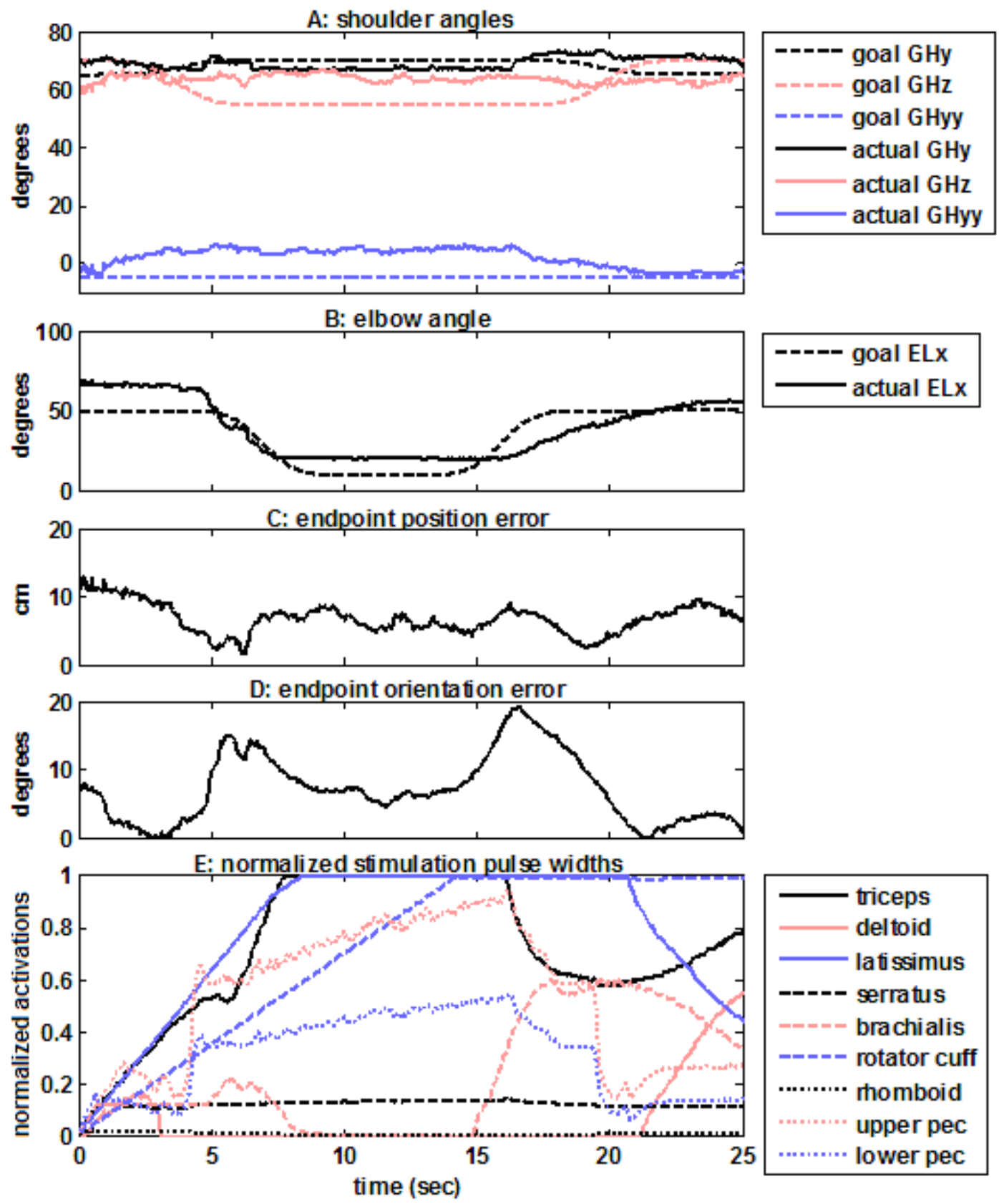

Figure 7-8: Example of a goal-oriented movement for the shoulder and elbow angles. Panels A and $\mathrm{B}$ show the goal and output angles for the shoulder and elbow, respectively. Panel $\mathrm{C}$ shows the endpoint position error, and panel D shows the endpoint orientation error. Panel E shows the stimulation patterns, normalized to the maximum pulse width of each muscle. 
The endpoint errors during the entire movement (left) and the movement "goal" (right) are shown in figure 7-9, for 15 goal-oriented movements. Panel A includes the position error, and panel B the orientation error.
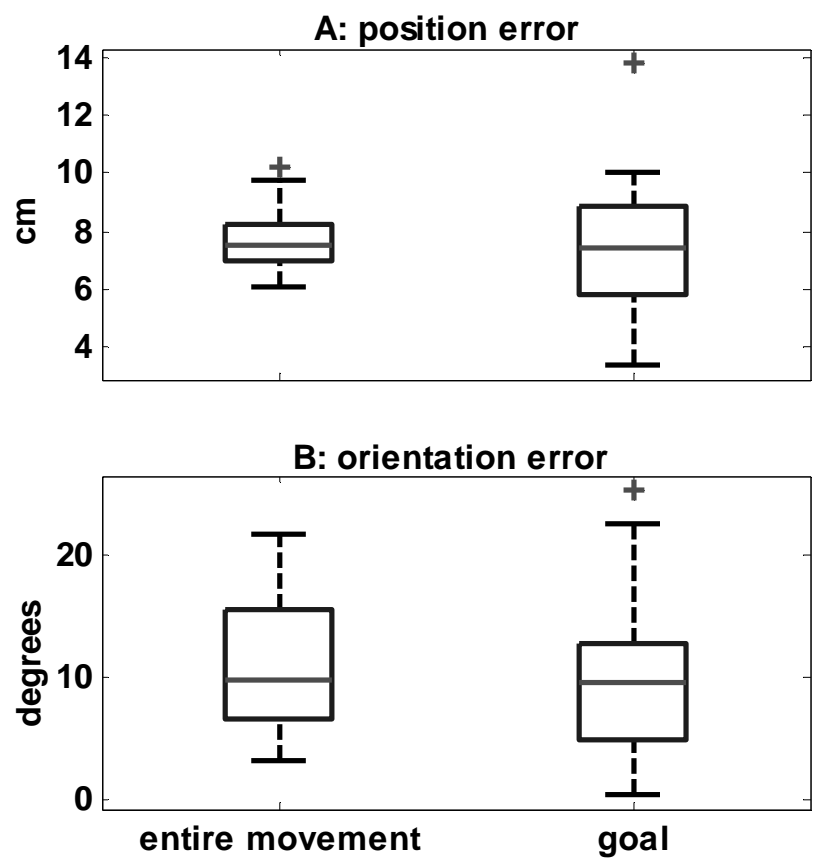

Figure 7-9: Summary of the shoulder-elbow controller performance from 15 goal-oriented movements. Panel A shows the endpoint position error, and panel B shows the endnoint orientation error.

Finally, figure 7-10 shows the results from the variability experiment. Each panel is sized according to the maximum range ever recorded for that degree of freedom (e.g. the maximum range for the humerus plane of elevation was 50 degrees). The left boxplot is the difference in the resulting motion from the same activation patterns, when the Optotrak markers were removed and then put on again. The middle boxplot is the difference when the arm was taken off the arm support and then put on again. The right boxplot is the difference 
recorded in the beginning and the end of an experimental session, during which the Optotrak markers and the arm support were not moved.
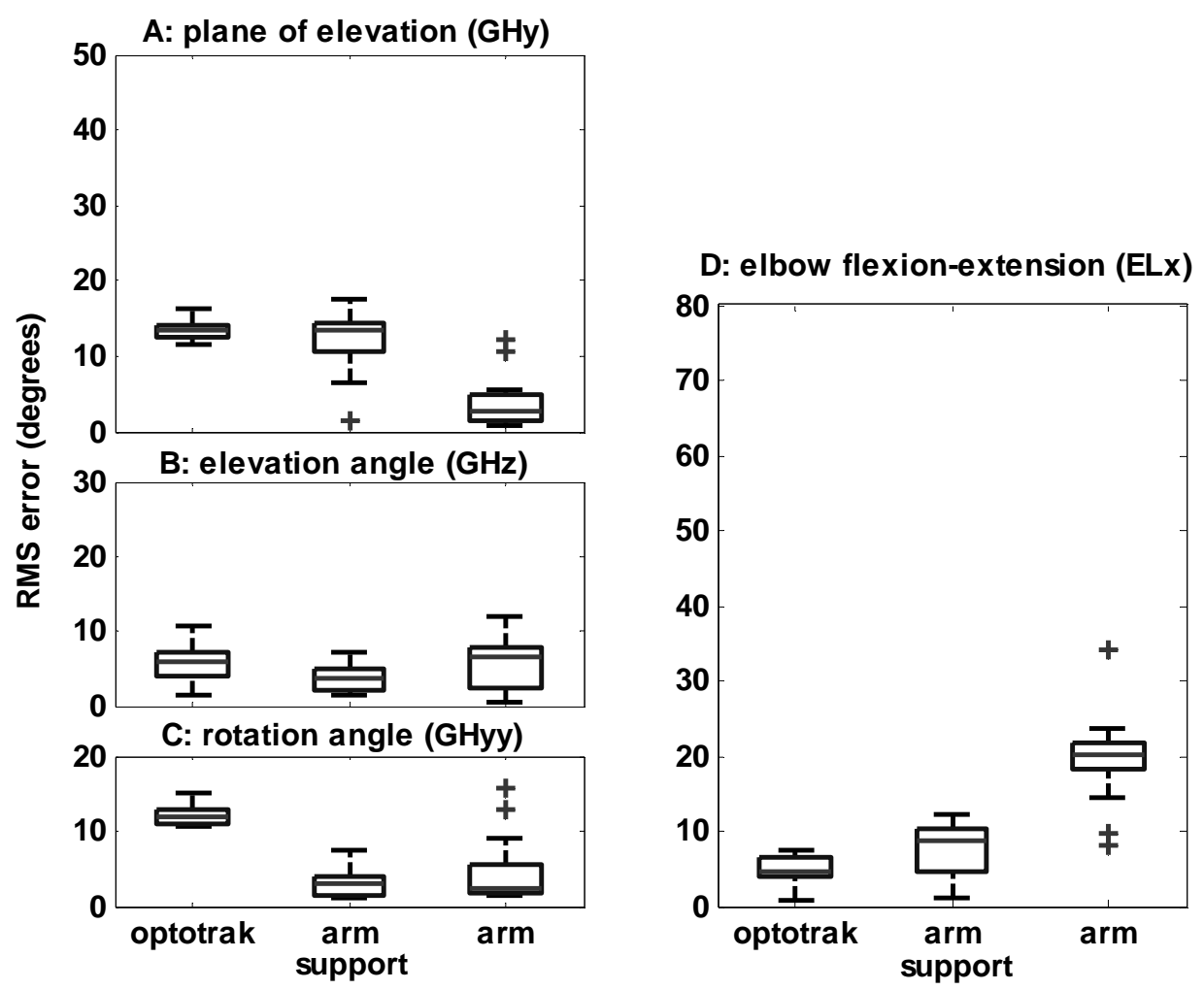

Figure 7-10: Variability due to the Optotrak markers (left boxplot), placement of the mobile arm support (middle) and arm stiffness (right boxplot). Each panel is scaled according to the maximum range of the specific degree of freedom. For example, panel $\mathrm{A}$ reaches 50 degrees because that was the maximum range recorded for the plane of elevation.

\section{Discussion}

A control design has been developed for restoration of shoulder and elbow function in high cervical level spinal cord injury. Knowledge of the arm dynamics is acquired through use of the system and stored in the artificial neural networks of the feedforward part of the controller. The Optotrak provides real time feedback of the arm position, used by the fuzzy 
PD part of the controller to adjust the stimulation patterns and allow the arm to follow the desired trajectory. After the required stimulation levels of the prime shoulder and elbow movers are determined, the scapular and glenohumeral stability muscles are added to ensure shoulder stability. Those are not dependent directly on the desired kinematics, but on the stimulation of the shoulder moving muscles, through model-based relationships. The controller was implemented in one subject with a high level upper extremity neuroprosthesis, using nine stimulation channels (seven for the shoulder and two for the elbow). It demonstrated good tracking performance of goal-oriented movements.

For the elbow flexion-extension angle, the controller achieved a tracking error of less than 10 degrees, and a goal error (i.e. error at the goal angle of the movement) of less than 5 degrees. This is only about $6 \%$ of the maximum range of 80 degrees. The good tracking performance was mostly due to the feedback controller, since the feedforward part by itself showed an error of about 20 degrees (figure 7-3). The feedforward controller was trained with data collected from the elbow of the subject, and the high error suggests that training was not very successful, probably because the training data were not consistent. This is supported by figure 7-10, which shows that the elbow angle had a variability of 20 degrees that was not explained by the Optotrak marker placement or the arm support, but was solely due to changes in the arm response to stimulation. It was observed during the experimental sessions that the arm of the subject showed varying degrees of spasticity, possibly related to the amount of exercise prior to the session. The fact that the elbow motion resulting from the same stimulation patterns was, at times, very different, hindered the performance of the feedforward controller. On the other hand, it clearly illustrated the need for a feedback controller, to correct the errors and allow the movements to be successfully performed. 
Similar results were obtained when the controller design was applied to the combined shoulder and elbow. The subject has significant shoulder muscle hypertonia, which is an abnormal increase in muscle tension, and results in a limited range of motion at the shoulder even with the highest stimulation levels ( 50 degrees for the plane of elevation, 30 degrees for the elevation angle, and 20 degrees for the rotation angle). Figure 7-10 also shows that there was considerable variability in the experimental setup, reaching $60 \%$ of the angle range in the case of humeral rotation. This is most likely due to the so-called "soft tissue artifact", which is the displacement between the Optotrak markers and the underlying bone due to the interposition of muscles and soft tissue (Cutti et al. 2006). It is a problem of all skin-mounted marker systems, and could be addressed in the future with the use of implanted sensors. In the meantime, our results show that for movements that do not require large humeral rotations, the use of feedback control produced an endpoint tracking error of less than $10 \mathrm{~cm}$ in position and 25 degrees in orientation. This error could be further reduced with stimulation of wrist muscles, which were not included in this study.

The relationship between the scapular and glenohumeral stability muscles, and the main shoulder moving muscles was based on model simulations. The model was customized to simulate the specific high tetraplegia subject with the FES system, so that the resulting activation patterns for shoulder stability would be as accurate as possible for this subject and not an able-bodied person. However, there are still many differences between the model and the actual subject, such as the exact force the muscles can produce, and the joint stiffness and spasticity. If it was necessary to further adapt the activation patterns, measurements could be made of the scapular orientation and estimates of the glenohumeral joint center in various arm positions, with different scapular and rotator cuff muscle stimulation levels. But as 
mentioned earlier, this subject has considerable stiffness at the shoulder, which minimizes the likelihood of instability.

The adaptation technique for the feedforward part of the controller was based on two pairs of antagonistic muscles (brachialis-triceps and deltoid-latissimus dorsi), and activation patterns between shoulder stabilizing and moving muscles. The same technique could be used for other sets of stimulated muscles, in different FES systems. Co-contraction between a pronator and the supinator muscle could be used for control of forearm pronation-supination similarly to elbow flexion-extension. If more stimulating channels were available for shoulder muscles, synergistic patterns might need to be implemented to facilitate control, for example all the available shoulder flexors might be constrained to activate at the same time. More muscles with multiple functions, crossing many degrees of freedom would pose a more challenging control problem, but would also allow for a wider range of restored movements. 


\section{Chapter 8: Conclusions}

The main focus of this study was controller design and implementation in a high level upper extremity FES system. The use of musculoskeletal modeling played an important part in both the development and the testing phase. An upper extremity model was first used to determine the optimal electrode set for a neuroprosthesis with a limited number of available stimulating channels. Subsequently, the combined feedforward - feedback controller approach was tested in increasingly complicated upper limb models. The first consisted of six muscles and two degrees of freedom and, in spite of the small scale, had an accurate representation of the non-linear muscle force production, redundancy, and kinematic coupling seen in the actual human arm. The second model included 22 shoulder and arm muscles and a complete glenohumeral joint, which allowed for testing of both the trajectory tracking and shoulder stabilizing ability of the controller. Finally, the controller was implemented in one subject with high level tetraplegia and an adaptation technique was developed to customize the controller parameters to the characteristics of the specific individual. In order to handle the redundancy of the upper limb, adaptation was performed using co-contraction of antagonistic muscles, and activation patterns among shoulder stabilizing and moving muscles. In simulation, the controller was found to have good tracking performance and ensure glenohumeral stability. When implemented in the subject, it achieved adaptation and allowed the execution of the desired movements.

Musculoskeletal models can be very useful tools in the development of neuroprosthetic systems. They can be used for design and testing in the place of timeconsuming trial-and-error techniques, and possibly invasive procedures. One application of 
modeling was presented in chapter 3 , where a large number of simulations were performed to determine which muscle and nerve cuff electrodes should be included in a high tetraplegia FES system in order to restore the most function. Without the model, this decision would have to be made based on the known actions of muscles and the experience and intuition of surgeons and technicians. This might have resulted in a successful system, but it is hard to imagine that it would have included optimal electrode placement, such as the nerve cuff electrode on the long thoracic nerve, which was placed to stimulate only the lower half of the serratus anterior, following the model-based recommendation.

Models were used throughout the rest of this study, not only for controller development, but also for the testing of adaptation techniques before they were implemented in the FES user. In chapters 2, 3 and 7, a model was customized to an individual with high level tetraplegia by setting the muscle force of paralyzed muscles to zero. Muscles stimulated by the FES system were allowed to produce up to $50 \%$ of able-bodied force, due to possible denervation and partial paralysis. In chapter 6, a hypothetical, "different" individual was modeled by reducing the muscle strength and increasing the muscle lengths. One of the greatest benefits of modeling is that it offers the flexibility of adjusting parameters to simulate different individuals. For example, different sets of "paralyzed" muscles would be used to describe the corresponding levels of spinal cord injury. In addition, models can be used to simulate interventions which are often combined with FES, such as tendon transfers. Finally, they could be used in the future to simulate other disorders such as stroke. FES has already been used in that population (Kralj et al. 1993; Burridge et al. 1997; Yu et al. 2001) and modeling studies could benefit the development of those systems as well. 
The feedforward-feedback controller design was introduced in chapter 4, and developed in the following chapters. This design was chosen in order to combine the advantages of the two control approaches. Feedforward control was implemented not in the form of pre-programmed stimulation patterns, which is quite limited in the number of movements it can produce, but as direct inverse control: the inverse of the system is used to control it, so that the desired system output becomes the controller input, and the required system input is calculated as the controller output. With this design, any desired motion within the workspace can be performed, if it is applied as the controller input, and not just a set of pre-defined movements. Another advantage of this feedforward controller is that it is trained on the inverse dynamics of the biomechanical system, and thus it can learn beneficial relationships among muscle stimulation levels and joint angles, such as patterns that minimize muscle fatigue, or ensure stability of highly mobile joints like the glenohumeral joint.

Without monitoring the system output, the controller cannot correct the stimulation patterns to account for external forces, muscle fatigue or inaccuracies in the description of the system inverse. One solution is to expect the FES user to adjust the controller command in the appropriate way to correct the movement errors. However, this becomes more difficult as the number of joints and multi-joint muscles increases, and the required command adjustment becomes less straightforward. This is true in the case of an FES system for high level tetraplegia, where most of the stimulated shoulder muscles cross multiple joints. For this reason, the chosen solution is to add a feedback loop, which automatically adjusts the stimulation patterns based on the movement error. Used by itself, feedback control can be slow, since an error needs to develop before a correction can be applied (Abbas and Chizeck 
1995). But combined with feedforward control, it only needs to adjust the stimulation patterns already calculated by the feedforward part, to improve the trajectory tracking performance.

Another advantage of the combined feedforward-feedback design, specific to upper extremity FES systems, was described in chapter 5 . Stability of the glenohumeral joint is an important issue for FES control, since it relies almost entirely on the activity of the surrounding rotator cuff muscles which, in the case of high tetraplegia, need to be appropriately stimulated by the FES system. Measurement of the joint center translation is not currently possible, so a method that does not rely on knowledge of the instability risk had to be developed. The combination of feedforward and feedback offers a qualitative assessment of the instability risk: under normal conditions, the stimulation levels are calculated mostly by the feedforward controller, and it is only when muscles fatigue or external disturbances are applied that the feedback generates significant stimulation adjustments. Those are the cases when instability is likely to occur, either because of the external force itself, or because of muscle reaction to that force. By monitoring the feedback contribution to the overall stimulation levels, the controller increases the rotator cuff activity when the chance of instability is suspected. The simulation study in chapter 5 shows that with this method, in the presence of various external forces, glenohumeral stability is always maintained.

Glenohumeral stability in the actual FES subject is discussed in chapter 7. The patterns of stimulation of the rotator cuff muscles learned by the feedforward controller are model-based. The function of these muscles is to keep the humeral head inside the glenoid, and thus they have little effect on the movement produced. Since their function cannot be 
measured, it is very difficult to adapt these model-based relationships to the specific FES user. Because of the differences between the model and the actual person, it cannot be certain that the model-based patterns are sufficient to guarantee glenohumeral stability. As mentioned in chapter 7, the specific subject had such high shoulder muscle hypertonia that instability was never an issue. If, in future subjects, stimulation of the rotator cuff muscles according to the modeling results does not protect against subluxation, measurements to estimate the joint center in various arm positions and stimulation levels might be used to adjust the patterns. Another option would be to apply constant rotator cuff stimulation, which has been shown to reduce subluxation in individuals with hemiplegia (Yu et al. 2004).

Adaptation of the FES controller to the needs of the specific user is important for the success of the system, whether it was model-based or developed through trial-and-error. The ability to adjust the controller parameters is very useful during the initial implementation phase, when the available muscle forces, possible joint stiffness and other individual characteristics first show how they affect the system performance. However, these characteristics could change with time., For example, muscles that suffered from disuse atrophy while paralyzed, become stronger once they get regularly stimulated through FES (Belanger et al. 2000). In a feedforward-feedback control scheme such changes could be successfully handled by the feedback part, but the advantages of the feedforward controller would be lost. Alternatively, continuing adaptation could adjust the feedforward to these long-term changes, allowing the system to keep the same level of performance.

Artificial neural networks are used to implement the feedforward controller. Their advantage is that they can be trained to approximate complicated functions, using only pairs of inputs and outputs. They achieve this by changing the weights in their network of 
processing elements, and thus they have the ideal structure for adaptation. However, the ANN parameters do not have a physiological meaning: the weight adjustment that results in a more accurate approximation of the inverse dynamics does not give any information on the reason for the adjustment. It could be that, in reality, a muscle is stronger or weaker than the modeling equivalent; or there could be a more fundamental discrepancy, such as a different length-dependence of muscle force production. A controller with a structure similar to an inverse model would have to be used to answer these questions. However, an inverse model is much more computationally intensive than an ANN, and could not easily be used for real time control. In addition, a more sophisticated adaptation method of the model parameters would be needed, that would most likely require more information than muscle excitation levels and joint angles. It is possible that, in the future, faster processing and feedback of more signals, with the use of implanted or even natural sensors, could make the inverse model a feasible feedforward controller. Adaptation to a specific individual could then increase our knowledge of biomechanical systems, and also result in more accurate models.

Feedforward controller adaptation using solely inputs and outputs has the disadvantage that is does not work well in the case of redundant systems, which is the norm in biomechanics. There are typically multiple muscles crossing every joint, which is especially useful in paralyzed individuals with reduced force capacity. For this reason, inverse models need to distribute the forces to the muscles through an optimization routine, a main reason why they are computationally intensive. The addition of the co-contraction level between antagonists, presented in chapter 6 , and activation patterns among shoulder stabilizing and moving muscles, discussed in chapter 7, offers a solution to this problem, though specific to the high level tetraplegia FES subject. Using different synergistic patterns 
or co-contraction of antagonistic groups instead of individual muscles, the same basic idea could be applied to other FES systems. Such patterns could also be implemented in the inverse dynamic model, to eliminate the need for optimization, and thus increase the speed and make it a better candidate for feedforward control.

Chapter 6 also mentioned the concept of variable co-contraction. Most simulation and experimental tests in that chapter used a controller with constant co-contraction, usually zero, but a simple example of a variable co-contraction input was included. It illustrated how the advantages of low co-contraction (fast response, fatigue-resistance) can be combined with those of high co-contraction (movement accuracy). In the specific example, which was a goal-oriented movement, the desired co-contraction level was increased with the movement deceleration, to improve the accuracy near the goal position. A more complicated function for the co-contraction level could be developed, possibly based on able-bodied co-contraction strategies. Thus, this new controller input, added originally only as a way to assist adaptation in redundant systems, could also help understand and implement more natural movement control.

Feedback control requires sensors to monitor the movement and appropriately adjust the muscle stimulation levels. In this study, feedback of the output trajectory was provided by the Optotrak system. Since it was a feasibility study for an FES controller design, the Optotrak was sufficient; however, a more portable system of sensors would be required to allow this controller design to be implemented in a take-home system. Inertial sensors, that are placed on a body segment and give 3D kinematic information, are becoming smaller and more light-weight, and would be the easiest to use in the initial implementation (Veltink et al 1998). If feedback becomes a standard part of an FES system, sensors could be implanted, to 
avoid the cosmetic issues of surface sensors and the annoyance and repeatability issues associated with everyday placement. In the future, another alternative could be natural sensors: nerve cuff electrodes could be used to record from sensory nerves, which have been shown to provide information about joint angles (Riso et al. 2000).

The FES controller developed in this study was tested extensively in simulation, and implemented in one subject, the first to receive an implanted neuroprosthesis for restoration of motor function of the entire upper limb. The subject uses a mobile arm support, and has a very limited workspace, mostly due to significant shoulder stiffness that the stimulation cannot overcome. However, without the FES system, the paralyzed arm would not move at all; even a little movement has made a tremendous difference in the subject's life. The goal of this study was to develop modeling, adaptation and feedback methods to help optimize the functional gain for present and future FES users. 


\section{References}

Abbas, J. J. \& Chizeck, H. J. (1995) Neural network control of functional neuromuscular stimulation systems: Computer simulation studies. IN IEEE Trans Biomed Eng, 42. $1117-27$.

Abbas, J. J. \& Triolo, R. J. (1997) Experimental evaluation of an adaptive feedforward controller for use in functional neuromuscular stimulation systems. IN IEEE Trans Rehabil Eng, 5. 12-22.

Acosta, A. M., Van der Helm, F. C. \& Kirsch, R. F. Feasibility of Restoring Shoulder and Elbow Function in High Level Tetraplegia: Minimum Muscle Set via Modeling. IN IEEE Trans. Neural Systems and Rehabilitation Engineering, in press.

Adamczyk, M. M. \& Crago, P. E. (2000) Simulated feedforward neural network coordination of hand grasp and wrist angle in a neuroprosthesis. IN IEEE Trans Rehabil Eng, 8. $297-304$.

Allinger, T.L., van den Bogert AJ (1997) Skating technique for the straights, based on the optimization of a simulation model. IN Occup Health Indust Med 36(6): 289.

Åström, K. J. \& Hägglund, T. (2001) The future of PID control. IN Control Engineering Practice, 9. 1163-1175.

Åström, K. J. \& Hägglund, T. (2004) Revisiting the Ziegler-Nichols step response method for PID control. IN Journal of Process Control, 14. 635-650.

Bélanger M., Stein R.B., Wheeler G.D., Gordon T., Leduc B. (2000) Electrical stimulation: can it increase muscle strength and reverse osteopenia in spinal cord injured individuals? IN Arch Phys Med Rehabil. 81(8):1090-8.

Burridge J, Taylor P, Hagan S, Swain I. (1997) Experience of clinical use of the Odstock dropped foot stimulator. IN Artif Organs. 21(3):254-60.

Charlton, I.W., Johnson G.R. (2000). An interactive musculoskeletal model of the upper limb. IN Proceedings of the 3rd Conference of the International Shoulder Group. Newcastle upon Tyne, UK.

Chang, G. C., Luh, J. J., Liao, G. D., Lai, J. S., Cheng, C. K., Kuo, B. L. \& Kuo, T. S. (1997) A neuro-control system for the knee joint position control with quadriceps stimulation. IN IEEE Trans Rehabil Eng, 5. 2-11.

Chizeck H. J., Kobetic R., Marsolais E. B., Abbas J. J., Donner I. H., Simon E. (1988) Control of functional neuromuscular stimulation systems for standing and locomotion in paraplegics. IN Proc. IEEE, 76: 1155-1165.

Crago, P. E., Lan, N., Veltink, P. H., Abbas, J. J. \& Kantor, C. (1996) New control strategies for neuroprosthetic systems. IN J Rehabil Res Dev, 33. 158-172.

Crago, P. E., Memberg, W. D., Usey, M. K., Keith, M. W., Kirsch, R. F., Chapman, G. J., Katorgi, M. A. \& Perreault, E. J. (1998) An elbow extension neuroprosthesis for individuals with tetraplegia. IN IEEE Trans Rehabil Eng, 6. 1-6.

Creasey G.H., Grill J.H., Korsten M., Betz R., Anderson R., Walter J. (2001) An implantable neuroprosthesis for restoring bladder and bowel control to patients with spinal cord injuries: a multicenter trial. IN Arch Phys Med Rehabil 82: 1512-19.

Cutti A.G., Cappello A, Davalli A. (2006) In vivo validation of a new technique that compensates for soft tissue artefact in the upper-arm: preliminary results. IN Clin Biomech (Bristol, Avon). 21 Suppl 1:S13-9. 
Davis, J. A., Triolo, R. J., Uhlir, J. P., Bhadra, N., Lissy, D. A., Nandurkar, S. \& Marsolais, E. B. (2001) Surgical technique for installing an eight-channel neuroprosthesis for standing. IN Clin Orthop Relat Res, . 237-252.

de Groot, J. H. \& Brand, R. (2001) A three-dimensional regression model of the shoulder rhythm. IN Clin Biomech (Bristol, Avon), 16. 735-743.

Delp, S.L. (1995) Computer modeling of movement abnormalities and their surgical corrections. IN Gait \& Posture 3(2): 106.

DeLuca, C.J., Forrest W.J. (1973). Force analysis of individual muscles acting simultaneously on the shoulder during isometric abduction. IN Journal of Biomechanics 6, 385-393.

Dul, J. (1987). Shoulder muscle load during work with elevated arms. IN Proceedings of the $11^{\text {th }}$ International Congress in Biomechanics. Amsterdam, the Netherlands.

Ferrarin, M., Palazzo, F., Riener, R. \& Quintern, J. (2001) Model-based control of FESinduced single joint movements. IN IEEE Trans Neural Syst Rehabil Eng, 9. 245257.

Fukunaga, T., Roy, R. R., Shellock, F. G., Hodgson, J. A., Day, M. K., Lee, P. L., KwongFu, H. \& Edgerton, V. R. (1992) Physiological cross-sectional area of human leg muscles based on magnetic resonance imaging. IN J Orthop Res, 10. 928-934.

Glenn, W. W. and M. L. Phelps (1985) Diaphragm pacing by electrical stimulation of the phrenic nerve. IN Neurosurgery 17(6): 974-84.

Gribble P.L., Mullin L.I., Cothros N. and Mattar A. (2003) Role of cocontraction in arm movement accuracy. IN Journal of Neurophysiology 89: 2396-2405.

Grill, W. M. \& Mortimer, J. T. (2000) Neural and connective tissue response to long-term implantation of multiple contact nerve cuff electrodes. IN J Biomed Mater Res, 50. $215-226$.

Handa, Y. and Hoshimiya N. (1987) Functional electrical stimulation for the control of the upper extremities. IN Med Prog Technol 12(1-2): 51-63.

Happee, R. (1994) Inverse dynamic optimization including muscular dynamics, a new simulation method applied to goal directed movements. IN J Biomech, 27. 953 - 960.

Happee, R. \& Van der Helm, F. C. (1995) The control of shoulder muscles during goal directed movements, an inverse dynamic analysis. IN J Biomech, 28. 1179-1191.

Heilman, B. P., Audu, M. L., Kirsch, R. F. \& Triolo, R. J. (2006) Selection of an optimal muscle set for a 16-channel standing neuroprosthesis using a human musculoskeletal model. IN J Rehabil Res Dev, 43. 273-286.

Hodges, P.W., Bui, B.H. (1996). A comparison of computer-based methods for the determination of onset of muscle contraction using electromyography. IN Electroencephalography and clinical Neurophysiology 101, 511 - 519.

Hogfors, C., Sigholm G., Herberts P. (1987). Biomechanical model of the human shoulderI. Elements. IN Journal of Biomechanics 20(2), 157-166.

Hoshimiya, N., Naito, A., Yajima, M. \& Handa, Y. (1989) A multichannel FES system for the restoration of motor functions in high spinal cord injury patients: A respirationcontrolled system for multijoint upper extremity. IN IEEE Trans Biomed Eng, 36. $754-60$.

Jenkins, D.B. (2002). Hollinshead's functional anatomy of the limbs and back. Saunders, $8^{\text {th }}$ edition. 
Jezernik S, Wassink RG, Keller T. (2004) Sliding mode closed-loop control of FES: controlling the shank movement. IN IEEE Trans Biomed Eng. 51(2):263-72.

Jonic S. \& Popovic, D. (1997) Machine learning for prediction of muscle activations for a rule-based controller. IN Engineering in Medicine and Biology society, 1997. Proceedings of the 19th Annual International Conference of the IEEE, 4. 1781-1784 vol.4.

Kameyama, J., Handa Y., Hoshimiya N., Sakurai M. (1999) Restoration of shoulder movement in quadriplegic and hemiplegic patients by functional electrical stimulation using percutaneous multiple electrodes. IN Tohoku J Exp Med 187(4): 329-37.

Karlsson, D., Peterson B. (1992). Towards a model for force predictions in the human shoulder. IN Journal of Biomechanics 25(2), 189-199.

Karniel A.; Inbar, G. F. (Feb 2000) Human motor control: Learning to control a timevarying, nonlinear, many-to-one system. IN Systems, Man and Cybernetics, Part C: Applications and Reviews, IEEE Transactions on, 30. 1-11.

Keith, M. W., Peckham, P. H., Thrope, G. B., Buckett, J. R., Stroh, K. C. \& Menger, V. (1988) Functional neuromuscular stimulation neuroprostheses for the tetraplegic hand. IN Clin Orthop Relat Res, . 25-33.

Keith, M.W., Lacey E.H. (1991). Surgical rehabilitation of the tetraplegic upper extremity. IN Journal of Neurologic Rehabilitation. 5, 75-87.

Kilgore, K. L., Peckham, P. H., Thrope, G. B., Keith, M. W. \& Gallaher-Stone, K. A. (1989) Synthesis of hand grasp using functional neuromuscular stimulation. IN IEEE Trans Biomed Eng, 36. 761-70.

Kilgore, K. L., Peckham, P. H., Keith, M. W., Montague, F. W., Hart, R. L., Gazdik, M. M., Bryden, A. M., Snyder, S. A. \& Stage, T. G. (2003) Durability of implanted electrodes and leads in an upper-limb neuroprosthesis. IN J Rehabil Res Dev, 40. 457-468.

Kim JY, Popovic MR, Mills JK. (2006) Dynamic modeling and torque estimation of FESassisted arm-free standing for paraplegics. IN IEEE Trans Neural Syst Rehabil Eng. 4(1):46-54.

Kirsch, R. F., Acosta, A. M., van der Helm, F. C., Rotteveel, R. J. \& Cash, L. A. (2001) Model-based development of neuroprostheses for restoring proximal arm function. IN J Rehabil Res Dev, 38. 619-626.

Klein Breteler, M. D., Spoor, C. W. \& Van der Helm, F. C. (1999) Measuring muscle and joint geometry parameters of a shoulder for modeling purposes. IN J Biomech, 32. $1191-1197$.

Kobetic, R., Marsolais, E.B. (1994). Synthesis of paraplegic gait with multichannel functional neuromuscular stimulation. IN IEEE Transactions in Rehabilitation Engineering. 2, 66-79.

Kralj A, Acimović R, Stanic U. (1993) Enhancement of hemiplegic patient rehabilitation by means of functional electrical stimulation. IN Prosthet Orthot Int. 17(2):107-14.

Lan, N., Crago, P. E. \& Chizeck, H. J. (1991) Control of end-point forces of a multijoint limb by functional neuromuscular stimulation. IN IEEE Trans Biomed Eng, 38. 953-65.

Lemay, M. A., P. E. Crago, Keith M.W. (1996). Restoration of pronosupination control by FNS in tetraplegia--experimental and biomechanical evaluation of feasibility. IN $J$ Biomech 29(4): 435-42. 
Lujan, J.L. (2007) Automated optimal coordination of multiple-degree-of-freedom musckuloskeletal actions in feedforward neuroprostheses. PhD Dissertation.

McLean, S. G., Su, A. \& van den Bogert, A. J. (2003) Development and validation of a 3-D model to predict knee joint loading during dynamic movement. IN J Biomech Eng, 125. 864-874.

Marsolais, E. B. and Kobetic R. (1987). Functional electrical stimulation for walking in paraplegia. IN J Bone Joint Surg Am 69(5): 728-33.

Memberg, W., Peckham, P. \& Keith, M. (1994) A surgically-implanted intramuscular electrode for an implantableneuromuscular stimulation system. IN Rehabilitation Engineering, IEEE Transactions on [see also IEEE Trans. on Neural Systems and Rehabilitation], 2. 80-91.

Naples, G. G., Mortimer, J. T., Scheiner, A. \& Sweeney, J. D. (1988) A spiral nerve cuff electrode for peripheral nerve stimulation. IN IEEE Trans Biomed Eng, 35. 905916.

Nathan, R. H. (1989) An FNS-based system for generating upper limb function in the C4 quadriplegic. IN Medical and Biological Engineering and Computing, 27. 549-556.

Nathan, R. H. \& Ohry, A. (1990) Upper limb functions regained in quadriplegia: A hybrid computerized neuromuscular stimulation system. IN Arch Phys Med Rehabil, 71. $415-21$.

Norgaard, M., Ravn, O., Poulsen, N. K. \& Hansen, L. K. Neural Networks for Modelling and Control of Dynamic Systems London, Springer-Verlag.

Peckham, P. H., Keith M.W. \& Freehafer A.A. (1988). Restoration of functional control by electrical stimulation in the upper extremity of the quadriplegic patient. IN $J$ Bone Joint Surg Am 70(1): 144-8.

Pedrocchi, A., Ferrante, S., De Momi, E. \& Ferrigno, G. (2006) Error mapping controller: A closed loop neuroprosthesis controlled by artificial neural networks. IN $J$ Neuroengineering Rehabil, 3. 25-25.

Popović D, Stojanović A, Pjanović A, Radosavljević S, Popović M, Jović S, Vulović D. (1999) Clinical evaluation of the bionic glove. IN Arch Phys Med Rehabil. Mar;80(3):299-304.

Praagman M., Veeger H.E., Chadwick E.K., Colier W.N., van der Helm F.C.T. (2003) Muscle oxygen consumption, determined by NIRS, in relation to external force and EMG. IN Journal of Biomechanics 36, 905-912.

Praagman, M., Chadwick, E. K., van der Helm, F. C. \& Veeger, H. E. J. (2006) The relationship between two different mechanical cost functions and muscle oxygen consumption. IN J Biomech, 39. 758-765.

Riener, R., Quintern, J. \& Schmidt, G. (1996) Biomechanical model of the human knee evaluated by neuromuscular stimulation. IN J Biomech, 29. 1157-1167.

Riess, J. and Abbas J.J. (2000) Adaptive neural network control of cyclic movements using functional neuromuscular stimulation. IN IEEE Trans Rehabil Eng 8(1): 42-52.

Riso R.R., Mosallaie F.K., Jensen W., Sinkjaer T. (2000) Nerve cuff recordings of muscle afferent activity from tibial and peroneal nerves in rabbit during passive ankle motion. IN IEEE Trans Rehabil Eng. 8(2):244-58.

Solomonow M, Baratta R, Bernardi M (1994) Surface and wire EMG crosstalk in neighboring muscles. IN Journal of Electromyography and Kinesiology. 4,131-42. 
Stolov, W., Clowers M. (1981) Handbook of Severe Disability. Washington, D.C. U.S. Government Printing Office.

Stroeve, S. (1996) Learning combined feedback and feedforward control of a musculoskeletal system. IN Biol Cybern, 75. 73-83.

Tsirakos D., Baltzopoulos V., Bartlett R. (1997) Inverse optimization: functional and physiological considerations related to the force-sharing problem. IN Critical Reviews in Biomedical Engineering. 25, 371-407.

van der Helm, F. C. (1994) A finite element musculoskeletal model of the shoulder mechanism. IN J Biomech, 27. 551-69.

van der Helm, C.F.T. (1997) A standardized protocol for motion recordings of the shoulder. IN Proceedings of the $1^{\text {st }}$ Conference of the International Shoulder Group. Delft, the Netherlands.

van der Helm, F.C.T., Chadwick E.K. (2002) A forward-dynamic shoulder and elbow model. IN Proceedings of the $4^{\text {th }}$ Conference of the International Shoulder Group. Cleveland, $\mathrm{OH}, \mathrm{USA}$.

Veeger, H. E., van der Helm F.C.T., can der Woude L.H., Pronk G.M., Rozendal R.H. (1991) Inertia and muscle contraction parameters for musculoskeletal modelling of the shoulder mechanism. IN Journal of Biomechanics 24, 615-29.

Veeger, H. E., Yu B., An K.N., Rozendal R.H. (1997) Parameters for modeling the upper extremity. IN Journal of Biomechanics 30, 647-52.

Veeger H.E., van der Helm F.C. (2007) Shoulder function: the perfect compromise between mobility and stability. IN J Biomech. 40(10):2119-29.

Veltink, P. H., Chizeck, H. J., Crago, P. E. \& el-Bialy, A. (1992) Nonlinear joint angle control for artificially stimulated muscle. IN IEEE Trans Biomed Eng, 39. 368 - 80.

Veltink, P.H., Sinkjaer, T., Baten, C.T.M., Bergveld, P., van der Spek, J., Haugland, M. (1998) Artificial and natural sensors in FES-assisted human movement control, IN Proceedings of the 20th Annual International Conference of the IEEE, vol.5, pp.2247-2250

Wilkenfeld A.J., Audu M.L., Triolo R.J. (2006) Feasibility of functional electrical stimulation for control of seated posture after spinal cord injury: A simulation study. IN J Rehabil Res Dev. 43(2):139-52.

Yoshida, N., Tomita, Y., Honda, S. \& Saitoh, E. (2002) Functional Neuromuscular Stimulation for articular angle control with an Inverse Dynamics Model tuned by a neural network. IN Ergonomics, 45. 649-662.

Yu, D. T., Chae J., Walker M.E., Hart R.L., Petroski G.F. (2001). "Comparing stimulationinduced pain during percutaneous (intramuscular) and transcutaneous neuromuscular electric stimulation for treating shoulder subluxation in hemiplegia." Arch Phys Med Rehabil 82(6): 756-60. 